\title{
11. NSW HOSPITALS
}

\section{Onset and augmentation of labour in selected hospitals}

Table 121 gives onset or augmentation of labour for individual hospitals where the number of reported deliveries exceeded 200 in 2005, totals for all hospitals within each health area, and the NSW total.

\section{TABLE 121}

ONSET AND AUGMENTATION OF LABOUR BY HOSPITAL, NSW 2005"

\begin{tabular}{|c|c|c|c|c|c|c|c|c|c|c|c|c|c|c|c|c|c|c|c|c|}
\hline \multirow[t]{3}{*}{ Health Area and Hospit } & \multirow{2}{*}{\multicolumn{2}{|c|}{ Spontaneous }} & \multirow{2}{*}{\multicolumn{2}{|c|}{$\begin{array}{c}\text { Spontaneous } \\
\text { augmented } \\
\text { with ARM }\end{array}$}} & \multirow{2}{*}{\multicolumn{2}{|c|}{$\begin{array}{l}\text { augmented } \\
\text { oxytocics- } \\
\text { prostagl. }\end{array}$}} & \multicolumn{6}{|c|}{ Onset and augmentation of labour } & \multirow{2}{*}{\multicolumn{2}{|c|}{$\begin{array}{c}\text { Induced } \\
\text { ARM+ } \\
\text { oxytocics- } \\
\text { prostagl. }\end{array}$}} & \multirow{2}{*}{\multicolumn{2}{|c|}{$\begin{array}{l}\text { Induced } \\
\text { other }\end{array}$}} & \multirow{2}{*}{\multicolumn{2}{|c|}{ Not stated }} & \multirow{2}{*}{\multicolumn{2}{|c|}{ TOTAL }} \\
\hline & & & & & & & $\begin{array}{l}\text { us No } \\
\text { d } \\
-\end{array}$ & Labour & $\begin{array}{r}\text { Ind } \\
\text { oxyt } \\
\text { pro }\end{array}$ & $\begin{array}{l}\text { duced } \\
\text { tocics- } \\
\text { stagl. }\end{array}$ & $\begin{array}{r}\text { Ind } \\
-\quad \text { Af } \\
\text { or }\end{array}$ & $\begin{array}{l}\text { uced } \\
\text { RM } \\
\text { nly }\end{array}$ & & & & & & & & \\
\hline & No. & $\%$ & No & $\%$ & No. & $\%$ & No & $\%$ & No. & $\%$ & No. & $\%$ & No. & $\%$ & No. & $\%$ & No. & $\%$ & No. & $\%$ \\
\hline \multicolumn{21}{|l|}{ Sydney South West } \\
\hline Canterbury & 901 & 58.1 & 0 & 0.0 & 172 & 11.1 & 199 & 12.8 & 268 & 17.3 & 11 & 0.7 & 0 & 0.0 & 1 & 0.1 & 0 & 0.0 & 1552 & 100.0 \\
\hline Royal Prince Alfred & 2306 & 0.8 & 0 & 0.0 & 616 & 13.6 & 631 & 13.9 & 368 & 8.1 & 46 & 1.0 & 565 & 512.4 & 8 & 0.2 & 0 & 0.0 & 4540 & 0.0 \\
\hline Fairfield & 876 & 1.7 & 87 & 5.1 & 192 & 11.3 & 209 & 12.3 & 96 & 5.7 & 4 & 0.2 & 219 & 9 12.9 & 10 & 0.6 & 0 & 0.0 & 1693 & 100.0 \\
\hline Liverpool & 1341 & 4.8 & 294 & 9.8 & 300 & 10.0 & 408 & 13.6 & 277 & 9.2 & 27 & 0.9 & 328 & B 10.9 & 21 & 0.7 & 0 & 0.0 & 2996 & 100.0 \\
\hline Camp & 1199 & 52.0 & 135 & 5.9 & 133 & 5.8 & 287 & 12.4 & 159 & 6.9 & 45 & 2.0 & 301 & 13.1 & 47 & 2.0 & 0 & 0.0 & 2306 & 100.0 \\
\hline $\begin{array}{l}\text { Bankstown-Lidcombe } \\
\text { Sydney Southwest }\end{array}$ & 1080 & 57.1 & 79 & 4.2 & 132 & 7.0 & 206 & 10.9 & 113 & 6.0 & 13 & 0.7 & 255 & 13.5 & 13 & 0.7 & 0 & 0.0 & 1891 & 100.0 \\
\hline & 299 & 25.4 & 91 & 7.7 & 152 & 12.9 & 226 & 19.2 & 112 & 9.5 & 23 & 2.0 & 272 & 23.1 & 3 & 0.3 & 0 & 0.0 & 1178 & 100.0 \\
\hline Bowral & 343 & 9.1 & 12 & 1.7 & 49 & 7.0 & 85 & 12.2 & 57 & 8.2 & 15 & 2.1 & 129 & 918.5 & 9 & 1.3 & 0 & 0.0 & 699 & 100.0 \\
\hline Othe & 45 & 66.2 & 7 & 10.3 & 3 & 4.4 & 0 & 0.0 & 3 & 4.4 & 4 & 5.9 & 6 & 8.8 & 0 & 0.0 & 0 & 0.0 & 68 & 100.0 \\
\hline ALL HOSPITALS & 8390 & 49.6 & 705 & 4.2 & 1749 & 10.3 & 2251 & 13.3 & 1453 & 8.6 & 188 & 1.1 & 2075 & 12.3 & 112 & 0.7 & 0 & 0.0 & 16923 & 100.0 \\
\hline \multicolumn{21}{|c|}{ South Eastern Sydney \& IIlawarra } \\
\hline St. George & 1309 & 56.8 & 110 & & 173 & & 242 & & 190 & & 32 & & 233 & 310.1 & 15 & & 0 & 0.0 & 2304 & 100.0 \\
\hline Sutherland & 56 & 53.2 & 23 & 2.2 & 73 & 7.0 & 117 & 11.2 & 74 & 7.1 & 13 & 1.2 & 184 & 417.6 & 6 & 0.6 & 0 & 0.0 & 1046 & 100.0 \\
\hline Royal Hospital for & & & & & & & & & & & & & & & & & & & & \\
\hline & 2033 & 52.8 & 70 & 1.8 & 266 & 6.9 & 656 & 17.1 & 292 & 7.6 & 59 & 1.5 & 445 & 511.6 & 26 & 0.7 & 0 & 0.0 & 3847 & 100.0 \\
\hline Hurstv & 181 & 20.3 & 82 & 9.2 & 123 & 13.8 & 230 & 25.8 & 46 & 5.2 & 15 & 1.7 & 215 & 524.1 & 0 & 0.0 & 0 & 0.0 & 892 & 100.0 \\
\hline Kar & 103 & 16.3 & 39 & 6.2 & 64 & 10.1 & 206 & 32.6 & 85 & 13.4 & 13 & 2.1 & 119 & 9 18.8 & 3 & 0.5 & 0 & 0.0 & 632 & 100.0 \\
\hline & 453 & 25.9 & 153 & 8.8 & 231 & 13.2 & 422 & 24.2 & 192 & 11.0 & 49 & 2.8 & 237 & 713.6 & 8 & 0.5 & 1 & 0.1 & 1746 & 100.0 \\
\hline Princ & 380 & 23.9 & 159 & 10.0 & 167 & 10.5 & 442 & 27.8 & 128 & 8.1 & 58 & 3.7 & 225 & 514.2 & 17 & 1.1 & 12 & 0.8 & 1588 & 100.0 \\
\hline Shoa & 447 & 55.4 & 16 & 2.0 & 31 & 3.8 & 171 & 21.2 & 56 & 6.9 & 8 & 1.0 & 69 & $\begin{array}{ll}9 & 8.6\end{array}$ & 9 & 1.1 & 0 & 0.0 & 807 & 100.0 \\
\hline & 661 & 9.2 & 425 & 18.8 & 303 & 13.4 & 223 & & 134 & & 36 & 1.6 & 479 & 21.2 & 3 & 0.1 & 0 & 0.0 & 2264 & 100.0 \\
\hline & 221 & & 80 & & 122 & 12.5 & 199 & 20.5 & 58 & 6.0 & 17 & 1 & 275 & & 1 & & 0 & & 973 & 0.0 \\
\hline & 42 & 8.8 & 0 & 0.0 & 5 & 5.8 & 12 & 14.0 & 10 & 11.6 & 0 & 0.0 & 14 & & 3 & 3.5 & 0 & .0 & 86 & 0.0 \\
\hline ALL HOSPITAL & 6386 & 39.5 & 1157 & 7.1 & 1558 & 9.6 & 2920 & 18.0 & 1265 & 7.8 & 300 & 1.9 & 2495 & 515.4 & 91 & 0.6 & 13 & 0.1 & 16185 & 100.0 \\
\hline \multicolumn{21}{|l|}{ Sydney West } \\
\hline & 699 & 7.8 & 49 & 4.1 & 100 & 8.3 & 112 & 9.3 & 72 & 6.0 & 5 & 0.4 & 168 & B 13.9 & 4 & 0.3 & 0 & 0.0 & 1209 & 100.0 \\
\hline & 1478 & 2.2 & 123 & 4.3 & 251 & 8.9 & 335 & 11.8 & 155 & & 23 & 0.8 & 464 & 416 & 5 & 0.2 & 0 & 0.0 & 2834 & 100.0 \\
\hline & & 2.1 & 8 & 3.0 & 13 & 4.8 & 24 & & 31 & 11.5 & 4 & 1 & 22 & & 0 & & 0 & 0.0 & 269 & 100.0 \\
\hline & & & & & & & & & & & 71 & & 557 & & 9 & & 0 & & 439 & 0.0 \\
\hline & & & 350 & 8.2 & 578 & 13.5 & 635 & & 266 & & 12 & & 640 & & 22 & & 0 & 0 & 266 & 0.0 \\
\hline & 95 & 22.9 & 114 & 8.8 & 120 & 9.3 & 217 & 16.8 & 90 & 7.0 & 13 & 1.0 & 438 & 334 & 3 & 0.2 & 0 & 0.0 & 1290 & 0.0 \\
\hline $\mathrm{H}$ & 443 & 51.6 & 36 & 4.2 & 16 & 1.9 & 119 & 13.9 & 118 & 13.7 & 30 & 3.5 & 96 & 611.2 & 1 & 0.1 & 0 & 0.0 & 859 & 100.0 \\
\hline & 260 & 27.6 & 106 & 11.3 & 70 & 7.4 & 204 & 21.7 & 81 & 8.6 & 17 & 1.8 & 201 & 121.3 & 3 & 0.3 & 0 & 0.0 & 942 & 100.0 \\
\hline & 446 & 25.0 & 168 & 9.4 & 282 & 15.8 & 334 & 18.7 & 149 & 8.4 & 14 & 0.8 & 378 & 321.2 & 13 & 0.7 & 0 & 0.0 & 1784 & 100.0 \\
\hline $\mathrm{O}$ & 78 & 32.1 & 36 & 14.8 & 25 & 10.3 & 40 & 16.5 & 37 & 15.2 & 6 & 2.5 & 21 & 18.6 & 0 & 0.0 & 0 & 0.0 & 243 & 100.0 \\
\hline ALL HOSPITALS & 7133 & 41.6 & 1198 & 7.0 & 1584 & 9.2 & 2562 & 15.0 & 1418 & 8.3 & 195 & 1.1 & 2985 & 17.4 & 60 & 0.4 & 0 & 0.0 & 17135 & 100.0 \\
\hline Northern Sydney \& Cen & itral Co & ast & & & & & & & & & & & & & & & & & & \\
\hline Gosford & 839 & 34.6 & 355 & 14.6 & 301 & 12.4 & 340 & 14.0 & 193 & 8.0 & 18 & 0.7 & 379 & 915.6 & 1 & 0.0 & 0 & 0.0 & 2426 & 100.0 \\
\hline lyong & 172 & 77.1 & 33 & 14.8 & 11 & 4.9 & 1 & 0.4 & 2 & 0.9 & 1 & 0.4 & 3 & 31.3 & 0 & 0.0 & 0 & 0.0 & 223 & 100.0 \\
\hline Orn & 463 & 51.5 & 27 & 3.0 & 77 & 8.6 & 149 & 16.6 & 63 & 7.0 & 8 & 0.9 & 111 & 112.3 & 1 & 0.1 & 0 & 0.0 & 899 & 100.0 \\
\hline & 340 & 51.4 & 17 & 2.6 & 44 & 6.7 & 103 & 15.6 & 54 & 8.2 & 10 & 1.5 & 92 & 213.9 & 1 & 0.2 & 0 & 0.0 & 661 & 100.0 \\
\hline & 295 & 46.0 & 20 & 3.1 & 76 & 11.8 & 85 & 13.2 & 45 & 7.0 & 2 & 0.3 & 119 & 918.5 & 0 & 0.0 & 0 & 0.0 & 642 & 100.0 \\
\hline & 1048 & 43.7 & 139 & 5.8 & 286 & 11.9 & 418 & 17.4 & 151 & 6.3 & 16 & 0.7 & 341 & 114.2 & 0 & 0.0 & 0 & 0.0 & 2399 & 100.0 \\
\hline $\mathrm{N}$ & & 27.3 & 168 & 7.6 & 252 & 11.4 & 630 & 28.4 & 195 & 8.8 & 67 & 3.0 & 299 & 9 13.5 & 3 & 0.1 & 0 & 0.0 & 2219 & 100.0 \\
\hline rivate & 764 & 29.9 & 117 & 4.6 & 296 & 11.6 & 767 & 30.0 & 98 & 3.8 & 50 & 2.0 & 445 & 17.4 & 18 & 0.7 & 0 & 0.0 & 2555 & 100.0 \\
\hline Sydne & 970 & 44.2 & 0 & 0.0 & 1 & 0.0 & 477 & 21.7 & 111 & 5.1 & 33 & 1.5 & 601 & 127.4 & 2 & 0.1 & 0 & 0.0 & 2195 & 100.0 \\
\hline North Gosford Private & 191 & 22.2 & 42 & 4.9 & 64 & 7.4 & 225 & 26.2 & 87 & 10.1 & 14 & 1.6 & 229 & 926.6 & 8 & 0.9 & 0 & 0.0 & 860 & 100.0 \\
\hline Other Area hospitals & 132 & 99.2 & 1 & 0.8 & 0 & 0.0 & 0 & 0.0 & 0 & 0.0 & 0 & 0.0 & 0 & 0.0 & 0 & 0.0 & 0 & 0.0 & 133 & 100.0 \\
\hline ALL HOSPITALS & 5819 & 38.3 & 919 & 6.0 & 1408 & 9.3 & 3195 & 21.0 & 999 & 6.6 & 219 & 1.4 & 2619 & 917.2 & 34 & 0.2 & 0 & 0.0 & 15212 & 100.0 \\
\hline
\end{tabular}




\section{TABLE 121 (continued)}

ONSET AND AUGMENTATION OF LABOUR BY HOSPITAL, NSW $2005^{\sharp}$

\begin{tabular}{|c|c|c|c|c|c|c|c|c|c|c|c|c|c|c|c|c|c|c|c|c|}
\hline \multirow[t]{3}{*}{ Health Area and Hospit } & \multirow{2}{*}{\multicolumn{2}{|c|}{ Spontaneous }} & \multirow{2}{*}{\multicolumn{2}{|c|}{$\begin{array}{l}\text { Spontaneous } \\
\text { augmented } \\
\text { with ARM }\end{array}$}} & \multicolumn{8}{|c|}{ Onset and augmentation of labour } & \multirow{2}{*}{\multicolumn{2}{|c|}{$\begin{array}{c}\text { Induced } \\
\text { ARM+ } \\
\text { oxytocics- } \\
\text { prostagl. }\end{array}$}} & \multirow{2}{*}{\multicolumn{2}{|c|}{$\begin{array}{l}\text { Induced } \\
\text { other"\#\# }\end{array}$}} & \multirow{2}{*}{\multicolumn{2}{|c|}{ Not stated }} & \multirow{2}{*}{\multicolumn{2}{|c|}{ TOTAL }} \\
\hline & & & & & $\begin{array}{l}\text { Spor } \\
\text { aug } \\
\text { oxy } \\
\text { pro }\end{array}$ & $\begin{array}{l}\text { thaneou } \\
\text { Imented } \\
\text { tocics- } \\
\text { stagl."\# }\end{array}$ & & Labour & $\begin{array}{r}\text { Ind } \\
\text { oxytc } \\
\text { pro }\end{array}$ & $\begin{array}{l}\text { luced } \\
\text { ocics- } \\
\text { octagl. }\end{array}$ & & $\begin{array}{l}\text { luced } \\
\text { RM } \\
\text { nly }\end{array}$ & & & & & & & & \\
\hline & No. & $\%$ & No. & $\%$ & No. & $\%$ & No. & $\%$ & No. & $\%$ & No. & $\%$ & No. & $\%$ & No. & $\%$ & No. & $\%$ & No. & $\%$ \\
\hline \multicolumn{21}{|l|}{ Hunter \& New England } \\
\hline Armidale & 123 & 30.4 & 69 & 17.0 & 29 & 7.2 & 55 & 13.6 & 50 & 12.3 & 10 & 2.5 & 68 & 16.8 & 1 & 0.2 & 0 & 0.0 & 405 & 100.0 \\
\hline Inverell & 55 & 22.7 & 23 & 9.5 & 34 & 14.0 & 44 & 18.2 & 31 & 12.8 & 8 & 3.3 & 45 & 18.6 & 2 & 0.8 & 0 & 0.0 & 242 & 100.0 \\
\hline Moree & 108 & 45.0 & 18 & 7.5 & 24 & 10.0 & 29 & 12.1 & 18 & 7.5 & 2 & 0.8 & 37 & 15.4 & 4 & 1.7 & 0 & 0.0 & 240 & 100.0 \\
\hline Tamworth Base & 254 & 32.1 & 133 & 16.8 & 64 & 8.1 & 113 & 14.3 & 69 & 8.7 & 19 & 2.4 & 136 & 17.2 & 4 & 0.5 & 0 & 0.0 & 792 & 100.0 \\
\hline Manning Base & 262 & 37.5 & 97 & 13.9 & 50 & 7.2 & 92 & 13.2 & 44 & 6.3 & 12 & 1.7 & 140 & 20.1 & 1 & 0.1 & 0 & 0.0 & 698 & 100.0 \\
\hline Maitland & 815 & 52.0 & 35 & 2.2 & 52 & 3.3 & 263 & 16.8 & 148 & 9.4 & 44 & 2.8 & 208 & 13.3 & 3 & 0.2 & 0 & 0.0 & 1568 & 100.0 \\
\hline Muswellbrook & 128 & 53.1 & 15 & 6.2 & 9 & 3.7 & 32 & 13.3 & 26 & 10.8 & 13 & 5.4 & 18 & 7.5 & 0 & 0.0 & 0 & 0.0 & 241 & 100.0 \\
\hline Belmont & 202 & 62.9 & 12 & 3.7 & 10 & 3.1 & 37 & 11.5 & 18 & 5.6 & 6 & 1.9 & 35 & 10.9 & 1 & 0.3 & 0 & 0.0 & 321 & 100.0 \\
\hline John Hunter & 1666 & 49.2 & 176 & 5.2 & 208 & 6.1 & 476 & 14.1 & 240 & 7.1 & 93 & 2.7 & 498 & 14.7 & 27 & 0.8 & 0 & 0.0 & 3384 & 100.0 \\
\hline Newcastle Private & 658 & 39.7 & 52 & 3.1 & 86 & 5.2 & 360 & 21.7 & 113 & 6.8 & 51 & 3.1 & 333 & 20.1 & 6 & 0.4 & 0 & 0.0 & 1659 & 100.0 \\
\hline Other Area hospitals & 318 & 36.4 & 106 & 12.1 & 60 & 6.9 & 114 & 13.1 & 134 & 15.3 & 25 & 2.9 & 114 & 13.1 & 2 & 0.2 & 0 & 0.0 & 873 & 100.0 \\
\hline ALL HOSPITALS & 4589 & 44.0 & 736 & 7.1 & 626 & 6.0 & 1615 & 15.5 & 891 & 8.5 & 283 & 2.7 & 1632 & 15.7 & 51 & 0.5 & 0 & 0.0 & 10423 & 100.0 \\
\hline \multicolumn{21}{|l|}{ North Coast } \\
\hline Coffs Harbour & 330 & 39.1 & 109 & 12.9 & 68 & 8.1 & 131 & 15.5 & 67 & 7.9 & 19 & 2.3 & 120 & 14.2 & 0 & 0.0 & 0 & 0.0 & 844 & 100.0 \\
\hline Grafton Base & 174 & 39.7 & 35 & 8.0 & 40 & 9.1 & 82 & 18.7 & 73 & 16.7 & 2 & 0.5 & 29 & 6.6 & 3 & 0.7 & 0 & 0.0 & 438 & 100.0 \\
\hline Kempsey & 134 & 44.2 & 36 & 11.9 & 10 & 3.3 & 29 & 9.6 & 57 & 18.8 & 9 & 3.0 & 26 & 8.6 & 2 & 0.7 & 0 & 0.0 & 303 & 100.0 \\
\hline Lismore Base & 534 & 42.5 & 157 & 12.5 & 102 & 8.1 & 186 & 14.8 & 96 & 7.6 & 40 & 3.2 & 139 & 11.1 & 2 & 0.2 & 0 & 0.0 & 1256 & 100.0 \\
\hline Murwillumbah & 146 & 36.2 & 32 & 7.9 & 48 & 11.9 & 65 & 16.1 & 49 & 12.2 & 11 & 2.7 & 52 & 12.9 & 0 & 0.0 & 0 & 0.0 & 403 & 100.0 \\
\hline Tweed Heads & 443 & 42.4 & 83 & 7.9 & 122 & 11.7 & 136 & 13.0 & 77 & 7.4 & 28 & 2.7 & 157 & 15.0 & 0 & 0.0 & 0 & 0.0 & 1046 & 100.0 \\
\hline Port Macquarie Base & 286 & 38.0 & 115 & 15.3 & 52 & 6.9 & 120 & 16.0 & 54 & 7.2 & 10 & 1.3 & 115 & 15.3 & 0 & 0.0 & 0 & 0.0 & 752 & 100.0 \\
\hline Other Area hospitals & 256 & 59.1 & 73 & 16.9 & 26 & 6.0 & 9 & 2.1 & 21 & 4.8 & 13 & 3.0 & 35 & 8.1 & 0 & 0.0 & 0 & 0.0 & 433 & 100.0 \\
\hline ALL HOSPITALS & 2303 & 42.1 & 640 & 11.7 & 468 & 8.5 & 758 & 13.8 & 494 & 9.0 & 132 & 2.4 & 673 & 12.3 & 7 & 0.1 & 0 & 0.0 & 5475 & 100.0 \\
\hline \multicolumn{21}{|l|}{ Greater Southern } \\
\hline Goulburn Base & 122 & 42.7 & 39 & 13.6 & 41 & 14.3 & 54 & 18.9 & 22 & 7.7 & 2 & 0.7 & 4 & 1.4 & 2 & 0.7 & 0 & 0.0 & 286 & 100.0 \\
\hline Moruya & 148 & 44.8 & 42 & 12.7 & 15 & 4.5 & 45 & 13.6 & 39 & 11.8 & 8 & 2.4 & 32 & 9.7 & 1 & 0.3 & 0 & 0.0 & 330 & 100.0 \\
\hline Queanbeyan & 139 & 50.2 & 24 & 8.7 & 12 & 4.3 & 29 & 10.5 & 56 & 20.2 & 2 & 0.7 & 15 & 5.4 & 0 & 0.0 & 0 & 0.0 & 277 & 100.0 \\
\hline Griffith Base & 230 & 51.8 & 28 & 6.3 & 21 & 4.7 & 74 & 16.7 & 43 & 9.7 & 17 & 3.8 & 30 & 6.8 & 1 & 0.2 & 0 & 0.0 & 444 & 100.0 \\
\hline Wagga Wagga Base & 325 & 45.9 & 91 & 12.9 & 43 & 6.1 & 94 & 13.3 & 64 & 9.0 & 23 & 3.2 & 66 & 9.3 & 2 & 0.3 & 0 & 0.0 & 708 & 100.0 \\
\hline Calvary, Wagga Wagga & 204 & 36.7 & 40 & 7.2 & 33 & 5.9 & 133 & 23.9 & 84 & 15.1 & 14 & 2.5 & 40 & 7.2 & 8 & 1.4 & 0 & 0.0 & 556 & 100.0 \\
\hline Other Area hospitals & 535 & 39.2 & 185 & 13.6 & 73 & 5.3 & 187 & 13.7 & 212 & 15.5 & 39 & 2.9 & 128 & 9.4 & 6 & 0.4 & 0 & 0.0 & 1365 & 100.0 \\
\hline ALL HOSPITALS & 1703 & 42.9 & 449 & 11.3 & 238 & 6.0 & 616 & 15.5 & 520 & 13.1 & 105 & 2.6 & 315 & 7.9 & 20 & 0.5 & 0 & 0.0 & 3966 & 100.0 \\
\hline \multicolumn{21}{|l|}{ Greater Western } \\
\hline Dubbo Base & 448 & 37.3 & 108 & 9.0 & 46 & 3.8 & 143 & 11.9 & 103 & 8.6 & 121 & 10.1 & 227 & 18.9 & 4 & 0.3 & 0 & 0.0 & 1200 & 100.0 \\
\hline Mudgee & 112 & 48.3 & 9 & 3.9 & 10 & 4.3 & 38 & 16.4 & 32 & 13.8 & 5 & 2.2 & 26 & 11.2 & 0 & 0.0 & 0 & 0.0 & 232 & 100.0 \\
\hline Bathurst Base & 301 & 51.7 & 32 & 5.5 & 28 & 4.8 & 106 & 18.2 & 76 & 13.1 & 12 & 2.1 & 27 & 4.6 & 0 & 0.0 & 0 & 0.0 & 582 & 100.0 \\
\hline Orange Base & 284 & 36.1 & 114 & 14.5 & 51 & & 130 & 16.5 & 46 & 5.8 & 27 & 3.4 & 133 & 16.9 & 2 & 0.3 & 0 & 0.0 & 787 & 100.0 \\
\hline Broken Hill Base & 143 & 53.0 & 23 & 8.5 & 10 & 3.7 & 35 & 13.0 & 40 & 14.8 & 0 & 0.0 & 18 & 6.7 & 1 & 0.4 & 0 & 0.0 & 270 & 100.0 \\
\hline Other Area hospitals & 308 & 48.1 & 62 & 9.7 & 34 & 5.3 & 98 & 15.3 & 80 & 12.5 & 8 & 1.3 & 48 & 7.5 & 2 & 0.3 & 0 & 0.0 & 640 & 100.0 \\
\hline ALL HOSPITALS & 1596 & 43.0 & 348 & 9.4 & 179 & 4.8 & 550 & 14.8 & 377 & 10.2 & 173 & 4.7 & 479 & 12.9 & 9 & 0.2 & 0 & 0.0 & 3711 & 00.0 \\
\hline TOTAL NSW & 38027 & 42.7 & 6152 & 6.9 & 7812 & 8.81 & 14467 & 16.2 & 7417 & 8.3 & 1595 & 1.81 & 13273 & 14.9 & 384 & 0.4 & 13 & 0.0 & 89140 & 100.0 \\
\hline
\end{tabular}




\section{Type of delivery in selected hospitals}

Table 122 gives type of delivery for individual hospitals where the number of reported confinements exceeded 200 in 2005, totals for all hospitals within each health area, and the NSW total.

\section{TABLE 122}

TYPE OF DELIVERY BY HOSPITAL, NSW 2005"

\begin{tabular}{|c|c|c|c|c|c|c|c|c|c|c|c|c|c|c|}
\hline \multirow[t]{3}{*}{ Health Area and Hospital } & \multirow{2}{*}{\multicolumn{2}{|c|}{$\begin{array}{l}\text { Normal } \\
\text { vaginal }\end{array}$}} & \multirow{2}{*}{\multicolumn{2}{|c|}{ Forceps }} & \multicolumn{4}{|c|}{ Type of delivery } & \multirow{2}{*}{\multicolumn{2}{|c|}{$\begin{array}{l}\text { Elective } \\
\text { caesarean } \\
\text { section }\end{array}$}} & \multirow{2}{*}{\multicolumn{2}{|c|}{$\begin{array}{l}\text { Emergency } \\
\text { caesarean } \\
\text { section\#\# }\end{array}$}} & \multirow{2}{*}{\multicolumn{2}{|c|}{ TOTAL }} \\
\hline & & & & & \multicolumn{2}{|c|}{$\begin{array}{l}\text { Vacuum } \\
\text { extraction }\end{array}$} & \multicolumn{2}{|c|}{$\begin{array}{l}\text { Vaginal } \\
\text { breech }\end{array}$} & & & & & & \\
\hline & No. & $\%$ & No. & $\%$ & No. & $\%$ & No. & $\%$ & No. & $\%$ & No. & $\%$ & No. & $\%$ \\
\hline \multicolumn{15}{|l|}{ Sydney South West } \\
\hline Canterbury & 1061 & 68.4 & 15 & 1.0 & 122 & 7.9 & 4 & 0.3 & 199 & 12.8 & 151 & 9.7 & 1552 & 100.0 \\
\hline Royal Prince Alfred & 2749 & 60.6 & 122 & 2.7 & 359 & 7.9 & 23 & 0.5 & 631 & 13.9 & 656 & 14.4 & 4540 & 100.0 \\
\hline Fairfield & 1263 & 74.6 & 6 & 0.4 & 96 & 5.7 & 4 & 0.2 & 209 & 12.3 & 115 & 6.8 & 1693 & 100.0 \\
\hline Liverpool & 2047 & 68.3 & 33 & 1.1 & 210 & 7.0 & 17 & 0.6 & 408 & 13.6 & 281 & 9.4 & 2996 & 100.0 \\
\hline Campbelltown & 1724 & 74.8 & 15 & 0.7 & 64 & 2.8 & 8 & 0.3 & 287 & 12.4 & 208 & 9.0 & 2306 & 100.0 \\
\hline Bankstown-Lidcombe & 1438 & 76.0 & 21 & 1.1 & 105 & 5.6 & 3 & 0.2 & 206 & 10.9 & 118 & 6.2 & 1891 & 100.0 \\
\hline Sydney Southwest Private & 661 & 56.1 & 20 & 1.7 & 153 & 13.0 & 2 & 0.2 & 226 & 19.2 & 116 & 9.8 & 1178 & 100.0 \\
\hline Bowral & 421 & 60.2 & 28 & 4.0 & 101 & 14.4 & 2 & 0.3 & 85 & 12.2 & 62 & 8.9 & 699 & 100.0 \\
\hline Other Area hospitals & 50 & 73.5 & 3 & 4.4 & 6 & 8.8 & 2 & 2.9 & 0 & 0.0 & 7 & 10.3 & 68 & 100.0 \\
\hline ALL HOSPITALS & 11414 & 67.4 & 263 & 1.6 & 1216 & 7.2 & 65 & 0.4 & 2251 & 13.3 & 1714 & 10.1 & 16923 & 100.0 \\
\hline \multicolumn{15}{|c|}{ South Eastern Sydney \&IIlawarra } \\
\hline St. George & 1453 & 63.1 & 73 & 3.2 & 226 & 9.8 & 4 & 0.2 & 242 & 10.5 & 306 & 13.3 & 2304 & 100.0 \\
\hline Sutherland & 694 & 66.3 & 15 & 1.4 & 107 & 10.2 & 3 & 0.3 & 117 & 11.2 & 110 & 10.5 & 1046 & 100.0 \\
\hline Royal Hospital for Women & 2048 & 53.2 & 213 & 5.5 & 358 & 9.3 & 23 & 0.6 & 656 & 17.1 & 549 & 14.3 & 3847 & 100.0 \\
\hline Hurstville Community & 395 & 44.3 & 30 & 3.4 & 116 & 13.0 & 2 & 0.2 & 230 & 25.8 & 119 & 13.3 & 892 & 100.0 \\
\hline Kareena Private & 208 & 32.9 & 91 & 14.4 & 39 & 6.2 & 1 & 0.2 & 206 & 32.6 & 87 & 13.8 & 632 & 100.0 \\
\hline St. George Private & 787 & 45.1 & 78 & 4.5 & 184 & 10.5 & 1 & 0.1 & 422 & 24.2 & 274 & 15.7 & 1746 & 100.0 \\
\hline Prince of Wales Private & 677 & 42.6 & 61 & 3.8 & 193 & 12.2 & 1 & 0.1 & 442 & 27.8 & 214 & 13.5 & 1588 & 100.0 \\
\hline Shoalhaven & 485 & 60.1 & 34 & 4.2 & 20 & 2.5 & 5 & 0.6 & 171 & 21.2 & 92 & 11.4 & 807 & 100.0 \\
\hline Wollongong & 1544 & 68.2 & 34 & 1.5 & 199 & 8.8 & 6 & 0.3 & 223 & 9.8 & 258 & 11.4 & 2264 & 100.0 \\
\hline Figtree Private & 522 & 53.6 & 13 & 1.3 & 158 & 16.2 & 0 & 0.0 & 199 & 20.5 & 81 & 8.3 & 973 & 100.0 \\
\hline Other Area hospitals & 62 & 72.1 & 2 & 2.3 & 3 & 3.5 & 0 & 0.0 & 12 & 14.0 & 7 & 8.1 & 86 & 100.0 \\
\hline ALL HOSPITALS & 8875 & 54.8 & 644 & 4.0 & 1603 & 9.9 & 46 & 0.3 & 2920 & 18.0 & 2097 & 13.0 & 16185 & 100.0 \\
\hline \multicolumn{15}{|l|}{ Sydney West } \\
\hline Auburn & 957 & 79.2 & 26 & 2.2 & 30 & 2.5 & 6 & 0.5 & 112 & 9.3 & 78 & 6.5 & 1209 & 100.0 \\
\hline Blacktown & 1946 & 68.7 & 87 & 3.1 & 167 & 5.9 & 8 & 0.3 & 335 & 11.8 & 291 & 10.3 & 2834 & 100.0 \\
\hline Blue Mountains & 192 & 71.4 & 2 & 0.7 & 24 & 8.9 & 2 & 0.7 & 24 & 8.9 & 25 & 9.3 & 269 & 100.0 \\
\hline Nepean & 2162 & 62.9 & 46 & 1.3 & 221 & 6.4 & 10 & 0.3 & 542 & 15.8 & 458 & 13.3 & 3439 & 100.0 \\
\hline Westmead & 2702 & 63.3 & 145 & 3.4 & 149 & 3.5 & 28 & 0.7 & 635 & 14.9 & 607 & 14.2 & 4266 & 100.0 \\
\hline The Hills Private & 727 & 56.4 & 166 & 12.9 & 64 & 5.0 & 4 & 0.3 & 217 & 16.8 & 112 & 8.7 & 1290 & 100.0 \\
\hline Hawkesbury & 569 & 66.2 & 29 & 3.4 & 27 & 3.1 & 1 & 0.1 & 119 & 13.9 & 114 & 13.3 & 859 & 100.0 \\
\hline Nepean Private & 460 & 48.8 & 55 & 5.8 & 68 & 7.2 & 4 & 0.4 & 204 & 21.7 & 151 & 16.0 & 942 & 100.0 \\
\hline Westmead Private & 927 & 52.0 & 160 & 9.0 & 112 & 6.3 & 4 & 0.2 & 334 & 18.7 & 247 & 13.8 & 1784 & 100.0 \\
\hline Other Area hospitals & 151 & 62.1 & 3 & 1.2 & 19 & 7.8 & 0 & 0.0 & 40 & 16.5 & 30 & 12.3 & 243 & 100.0 \\
\hline ALL HOSPITALS & 10793 & 63.0 & 719 & 4.2 & 881 & 5.1 & 67 & 0.4 & 2562 & 15.0 & 2113 & 12.3 & 17135 & 100.0 \\
\hline \multicolumn{15}{|c|}{ Northern Sydney \& Central Coast } \\
\hline Gosford & 1436 & 59.2 & 25 & 1.0 & 236 & 9.7 & 9 & 0.4 & 340 & 14.0 & 380 & 15.7 & 2426 & 100.0 \\
\hline Wyong & 209 & 93.7 & 0 & 0.0 & 9 & 4.0 & 0 & 0.0 & 1 & 0.4 & 4 & 1.8 & 223 & 100.0 \\
\hline Hornsby & 551 & 61.3 & 41 & 4.6 & 57 & 6.3 & 4 & 0.4 & 149 & 16.6 & 97 & 10.8 & 899 & 100.0 \\
\hline Manly & 415 & 62.8 & 19 & 2.9 & 53 & 8.0 & 4 & 0.6 & 103 & 15.6 & 67 & 10.1 & 661 & 100.0 \\
\hline Mona Vale & 381 & 59.3 & 8 & 1.2 & 81 & 12.6 & 2 & 0.3 & 85 & 13.2 & 85 & 13.2 & 642 & 100.0 \\
\hline Royal North Shore & 1357 & 56.6 & 140 & 5.8 & 122 & 5.1 & 9 & 0.4 & 418 & 17.4 & 353 & 14.7 & 2399 & 100.0 \\
\hline Mater, North Sydney & 942 & 42.5 & 75 & 3.4 & 278 & 12.5 & 0 & 0.0 & 630 & 28.4 & 294 & 13.2 & 2219 & 100.0 \\
\hline North Shore Private & 1054 & 41.3 & 92 & 3.6 & 270 & 10.6 & 5 & 0.2 & 767 & 30.0 & 367 & 14.4 & 2555 & 100.0 \\
\hline Sydney Adventist & 1192 & 54.3 & 114 & 5.2 & 136 & 6.2 & 6 & 0.3 & 477 & 21.7 & 270 & 12.3 & 2195 & 100.0 \\
\hline North Gosford Private & 372 & 43.3 & 21 & 2.4 & 121 & 14.1 & 0 & 0.0 & 225 & 26.2 & 121 & 14.1 & 860 & 100.0 \\
\hline Other Area hospitals & 133 & 100.0 & 0 & 0.0 & 0 & 0.0 & 0 & 0.0 & 0 & 0.0 & 0 & 0.0 & 133 & 100.0 \\
\hline ALL HOSPITALS & 8042 & 52.9 & 535 & 3.5 & 1363 & 9.0 & 39 & 0.3 & 3195 & 21.0 & 2038 & 13.4 & 15212 & 100.0 \\
\hline
\end{tabular}


TABLE 122 (continued)

TYPE OF DELIVERY BY HOSPITAL, NSW 2005"

\begin{tabular}{|c|c|c|c|c|c|c|c|c|c|c|c|c|c|c|}
\hline \multirow[t]{3}{*}{ Health Area and Hospital } & \multirow{2}{*}{\multicolumn{2}{|c|}{$\begin{array}{l}\text { Normal } \\
\text { vaginal }\end{array}$}} & \multirow{2}{*}{\multicolumn{2}{|c|}{ Forceps }} & \multicolumn{4}{|c|}{ Type of delivery } & \multirow{2}{*}{\multicolumn{2}{|c|}{$\begin{array}{l}\text { Elective } \\
\text { caesarean } \\
\text { section }\end{array}$}} & \multirow{2}{*}{\multicolumn{2}{|c|}{$\begin{array}{c}\text { Emergency } \\
\text { caesarean } \\
\text { section\#\# }\end{array}$}} & \multirow{2}{*}{\multicolumn{2}{|c|}{ TOTAL }} \\
\hline & & & & & \multicolumn{2}{|c|}{$\begin{array}{l}\text { Vacuum } \\
\text { extraction }\end{array}$} & \multicolumn{2}{|c|}{$\begin{array}{l}\text { Vaginal } \\
\text { breech }\end{array}$} & & & & & & \\
\hline & No. & $\%$ & No. & $\%$ & No. & $\%$ & No. & $\%$ & No. & $\%$ & No. & $\%$ & No. & $\%$ \\
\hline \multicolumn{15}{|l|}{ Hunter \& New England } \\
\hline Armidale & 283 & 69.9 & 23 & 5.7 & 9 & 2.2 & 2 & 0.5 & 55 & 13.6 & 33 & 8.1 & 405 & 100.0 \\
\hline Inverell & 149 & 61.6 & 18 & 7.4 & 0 & 0.0 & 0 & 0.0 & 44 & 18.2 & 31 & 12.8 & 242 & 100.0 \\
\hline Moree & 166 & 69.2 & 4 & 1.7 & 13 & 5.4 & 1 & 0.4 & 29 & 12.1 & 27 & 11.3 & 240 & 100.0 \\
\hline Tamworth Base & 511 & 64.5 & 9 & 1.1 & 41 & 5.2 & 3 & 0.4 & 113 & 14.3 & 115 & 14.5 & 792 & 100.0 \\
\hline Manning Base & 466 & 66.8 & 5 & 0.7 & 51 & 7.3 & 3 & 0.4 & 92 & 13.2 & 81 & 11.6 & 698 & 100.0 \\
\hline Maitland & 1028 & 65.6 & 24 & 1.5 & 69 & 4.4 & 8 & 0.5 & 263 & 16.8 & 176 & 11.2 & 1568 & 100.0 \\
\hline Muswellbrook & 160 & 66.4 & 1 & 0.4 & 23 & 9.5 & 0 & 0.0 & 32 & 13.3 & 25 & 10.4 & 241 & 100.0 \\
\hline Belmont & 249 & 77.6 & 3 & 0.9 & 13 & 4.0 & 0 & 0.0 & 37 & 11.5 & 19 & 5.9 & 321 & 100.0 \\
\hline John Hunter & 2203 & 65.1 & 125 & 3.7 & 204 & 6.0 & 33 & 1.0 & 476 & 14.1 & 343 & 10.1 & 3384 & 100.0 \\
\hline Newcastle Private & 819 & 49.4 & 71 & 4.3 & 172 & 10.4 & 4 & 0.2 & 360 & 21.7 & 233 & 14.0 & 1659 & 100.0 \\
\hline Other Area hospitals & 615 & 70.4 & 29 & 3.3 & 48 & 5.5 & 2 & 0.2 & 114 & 13.1 & 65 & 7.4 & 873 & 100.0 \\
\hline ALL HOSPITALS & 6649 & 63.8 & 312 & 3.0 & 643 & 6.2 & 56 & 0.5 & 1615 & 15.5 & 1148 & 11.0 & 10423 & 100.0 \\
\hline \multicolumn{15}{|l|}{ North Coast } \\
\hline Coffs Harbour & 558 & 66.1 & 19 & 2.3 & 40 & 4.7 & 1 & 0.1 & 131 & 15.5 & 95 & 11.3 & 844 & 100.0 \\
\hline Grafton Base & 262 & 59.8 & 9 & 2.1 & 24 & 5.5 & 2 & 0.5 & 82 & 18.7 & 59 & 13.5 & 438 & 100.0 \\
\hline Kempsey & 237 & 78.2 & 1 & 0.3 & 5 & 1.7 & 0 & 0.0 & 29 & 9.6 & 31 & 10.2 & 303 & 100.0 \\
\hline Lismore Base & 814 & 64.8 & 32 & 2.5 & 38 & 3.0 & 4 & 0.3 & 186 & 14.8 & 182 & 14.5 & 1256 & 100.0 \\
\hline Murwillumbah & 230 & 57.1 & 5 & 1.2 & 31 & 7.7 & 0 & 0.0 & 65 & 16.1 & 72 & 17.9 & 403 & 100.0 \\
\hline Tweed Heads & 711 & 68.0 & 12 & 1.1 & 53 & 5.1 & 7 & 0.7 & 136 & 13.0 & 127 & 12.1 & 1046 & 100.0 \\
\hline Port Macquarie Base & 481 & 64.0 & 24 & 3.2 & 47 & 6.3 & 1 & 0.1 & 120 & 16.0 & 79 & 10.5 & 752 & 100.0 \\
\hline Other Area hospitals & 373 & 86.1 & 9 & 2.1 & 32 & 7.4 & 3 & 0.7 & 9 & 2.1 & 7 & 1.6 & 433 & 100.0 \\
\hline ALL HOSPITALS & 3666 & 67.0 & 111 & 2.0 & 270 & 4.9 & 18 & 0.3 & 758 & 13.8 & 652 & 11.9 & 5475 & 100.0 \\
\hline \multicolumn{15}{|l|}{ Greater Southern } \\
\hline Goulburn Base & 161 & 56.3 & 35 & 12.2 & 10 & 3.5 & 0 & 0.0 & 54 & 18.9 & 26 & 9.1 & 286 & 100.0 \\
\hline Moruya & 221 & 67.0 & 11 & 3.3 & 25 & 7.6 & 3 & 0.9 & 45 & 13.6 & 25 & 7.6 & 330 & 100.0 \\
\hline Queanbeyan & 208 & 75.1 & 3 & 1.1 & 13 & 4.7 & 0 & 0.0 & 29 & 10.5 & 24 & 8.7 & 277 & 100.0 \\
\hline Griffith Base & 291 & 65.5 & 15 & 3.4 & 18 & 4.1 & 2 & 0.5 & 74 & 16.7 & 44 & 9.9 & 444 & 100.0 \\
\hline Wagga Wagga Base & 453 & 64.0 & 25 & 3.5 & 42 & 5.9 & 5 & 0.7 & 94 & 13.3 & 89 & 12.6 & 708 & 100.0 \\
\hline Calvary, Wagga Wagga & 272 & 48.9 & 26 & 4.7 & 54 & 9.7 & 1 & 0.2 & 133 & 23.9 & 70 & 12.6 & 556 & 100.0 \\
\hline Other Area hospitals & 937 & 68.6 & 22 & 1.6 & 85 & 6.2 & 3 & 0.2 & 187 & 13.7 & 131 & 9.6 & 1365 & 100.0 \\
\hline ALL HOSPITALS & 2543 & 64.1 & 137 & 3.5 & 247 & 6.2 & 14 & 0.4 & 616 & 15.5 & 409 & 10.3 & 3966 & 100.0 \\
\hline \multicolumn{15}{|l|}{ Greater Western } \\
\hline Dubbo Base & 860 & 71.7 & 45 & 3.8 & 25 & 2.1 & 10 & 0.8 & 143 & 11.9 & 117 & 9.8 & 1200 & 100.0 \\
\hline Mudgee & 155 & 66.8 & 0 & 0.0 & 17 & 7.3 & 0 & 0.0 & 38 & 16.4 & 22 & 9.5 & 232 & 100.0 \\
\hline Bathurst Base & 345 & 59.3 & 8 & 1.4 & 29 & 5.0 & 1 & 0.2 & 106 & 18.2 & 93 & 16.0 & 582 & 100.0 \\
\hline Orange Base & 474 & 60.2 & 25 & 3.2 & 48 & 6.1 & 4 & 0.5 & 130 & 16.5 & 106 & 13.5 & 787 & 100.0 \\
\hline Broken Hill Base & 194 & 71.9 & 2 & 0.7 & 10 & 3.7 & 0 & 0.0 & 35 & 13.0 & 29 & 10.7 & 270 & 100.0 \\
\hline Other Area hospitals & 448 & 70.0 & $\overline{0}$ & 0.0 & 20 & 3.1 & 2 & 0.3 & 98 & 15.3 & 72 & 11.3 & 640 & 100.0 \\
\hline ALL HOSPITALS & 2476 & 66.7 & 80 & 2.2 & 149 & 4.0 & 17 & 0.5 & 550 & 14.8 & 439 & 11.8 & 3711 & 100.0 \\
\hline TOTAL NSW & 54568 & 61.2 & 2801 & 3.1 & 6372 & 7.1 & 322 & 0.4 & 14467 & 16.2 & 10610 & 11.9 & 89140 & 100.0 \\
\hline
\end{tabular}




\section{Pain relief in selected hospitals}

Table 123 gives type of pain relief provided to women for individual hospitals where the number of reported confinements exceeded 200 in 2005, totals for all hospitals within each health area and the NSW total. In addition to the types of pain relief listed a further 24,834 (27.9 per cent) women were reported to have received local anaesthetic to the perineum, and 6,147 (6.9 per cent) received a pudendal block.

\section{TABLE 123}

PAIN RELIEF BY HOSPITAL, NSW 2005*

\begin{tabular}{|c|c|c|c|c|c|c|c|c|c|c|c|c|c|c|}
\hline \multirow[t]{3}{*}{ Health Area and Hospital } & \multirow{2}{*}{\multicolumn{2}{|c|}{ Epidural }} & \multirow{2}{*}{\multicolumn{2}{|c|}{$\begin{array}{c}\text { General } \\
\text { anaesthetic }\end{array}$}} & \multicolumn{4}{|c|}{ Type of pain relief } & \multirow{2}{*}{\multicolumn{2}{|c|}{ Spinal }} & \multirow{2}{*}{\multicolumn{2}{|c|}{ Nil }} & \multirow{2}{*}{\multicolumn{2}{|c|}{ TOTAL }} \\
\hline & & & & & & $\begin{array}{l}\text { M } \\
\text { otics }\end{array}$ & & $\begin{array}{l}\text { ous } \\
\text { de }\end{array}$ & & & & & & \\
\hline & No. & $\%$ & No. & $\%$ & No. & $\%$ & No. & $\%$ & No. & $\%$ & No. & $\%$ & No. & $\%$ \\
\hline \multicolumn{15}{|l|}{ Sydney South West } \\
\hline Canterbury & 231 & 14.9 & 95 & 6.1 & 448 & 28.9 & 631 & 40.7 & 197 & 12.7 & 204 & 13.1 & 1552 & 100.0 \\
\hline Royal Prince Alfred & 1516 & 33.4 & 242 & 5.3 & 0 & 0.0 & 1899 & 41.8 & 715 & 15.7 & 514 & 11.3 & 4540 & 100.0 \\
\hline Fairfield & 38 & 2.2 & 181 & 10.7 & 479 & 28.3 & 781 & 46.1 & 143 & 8.4 & 283 & 16.7 & 1693 & 100.0 \\
\hline Liverpool & 548 & 18.3 & 193 & 6.4 & 1190 & 39.7 & 1345 & 44.9 & 412 & 13.8 & 263 & 8.8 & 2996 & 100.0 \\
\hline Campbelltown & 230 & 10.0 & 131 & 5.7 & 736 & 31.9 & 1306 & 56.6 & 344 & 14.9 & 294 & 12.7 & 2306 & 100.0 \\
\hline Bankstown/Lidcombe & 118 & 6.2 & 100 & 5.3 & 381 & 20.1 & 1072 & 56.7 & 214 & 11.3 & 251 & 13.3 & 1891 & 100.0 \\
\hline Sydney Southwest Private & te 129 & 11.0 & 52 & 4.4 & 409 & 34.7 & 702 & 59.6 & 191 & 16.2 & 36 & 3.1 & 1178 & 100.0 \\
\hline Bowral & 169 & 24.2 & 25 & 3.6 & 281 & 40.2 & 334 & 47.8 & 75 & 10.7 & 58 & 8.3 & 699 & 100.0 \\
\hline Other Area hospitals & 2 & 2.9 & 1 & 1.5 & 17 & 25.0 & 42 & 61.8 & 5 & 7.4 & 13 & 19.1 & 68 & 100.0 \\
\hline ALL HOSPITALS & 2981 & 17.6 & 1020 & 6.0 & 3941 & 23.3 & 8112 & 47.9 & 2296 & 13.6 & 1916 & 11.3 & 16923 & 100.0 \\
\hline \multicolumn{15}{|c|}{ South Eastern Sydney \&lllawarra } \\
\hline St. George & 667 & 28.9 & 73 & 3.2 & 362 & 15.7 & 1009 & 43.8 & 333 & 14.5 & 318 & 13.8 & 2304 & 100.0 \\
\hline Sutherland & 332 & 31.7 & 31 & 3.0 & 93 & 8.9 & 472 & 45.1 & 168 & 16.1 & 113 & 10.8 & 1046 & 100.0 \\
\hline Royal Hospital for Women & n2090 & 54.3 & 57 & 1.5 & 533 & 13.9 & 1152 & 29.9 & 647 & 16.8 & 378 & 9.8 & 3847 & 100.0 \\
\hline Hurstville Community & 225 & 25.2 & 25 & 2.8 & 53 & 5.9 & 257 & 28.8 & 162 & 18.2 & 39 & 4.4 & 892 & 100.0 \\
\hline Kareena Private & 160 & 25.3 & 15 & 2.4 & 15 & 2.4 & 102 & 16.1 & 134 & 21.2 & 25 & 4.0 & 632 & 100.0 \\
\hline St. George Private & 542 & 31.0 & 61 & 3.5 & 121 & 6.9 & 702 & 40.2 & 188 & 10.8 & 72 & 4.1 & 1746 & 100.0 \\
\hline Prince of Wales Private & 625 & 39.4 & 18 & 1.1 & 54 & 3.4 & 415 & 26.1 & 76 & 4.8 & 71 & 4.5 & 1588 & 100.0 \\
\hline Shoalhaven & 78 & 9.7 & 36 & 4.5 & 208 & 25.8 & 329 & 40.8 & 230 & 28.5 & 92 & 11.4 & 807 & 100.0 \\
\hline Wollongong & 477 & 21.1 & 96 & 4.2 & 425 & 18.8 & 1351 & 59.7 & 292 & 12.9 & 247 & 10.9 & 2264 & 100.0 \\
\hline Figtree Private & 71 & 7.3 & 31 & 3.2 & 73 & 7.5 & 435 & 44.7 & 198 & 20.3 & 40 & 4.1 & 973 & 100.0 \\
\hline Other Area hospitals & 8 & 9.3 & 1 & 1.2 & 18 & 20.9 & 15 & 17.4 & 16 & 18.6 & 23 & 26.7 & 86 & 100. \\
\hline ALL HOSPITALS & 5275 & 32.6 & 444 & 2.7 & 1955 & 12.1 & 6239 & 38.5 & 2444 & 15.1 & 1418 & 8.8 & 16185 & 100.0 \\
\hline \multicolumn{15}{|l|}{ Sydney West } \\
\hline Auburn & 99 & 8.2 & 81 & 6.7 & 254 & 21.0 & 589 & 48.7 & 90 & 7.4 & 275 & 22.7 & 1209 & 100.0 \\
\hline Blacktown & 583 & 20.6 & 147 & 5.2 & 561 & 19.8 & 1412 & 49.8 & 376 & 13.3 & 364 & 12.8 & 2834 & 100.0 \\
\hline Blue Mountains & 42 & 15.6 & 7 & 2.6 & 57 & 21.2 & 122 & 45.4 & 37 & 13.8 & 45 & 16.7 & 269 & 100.0 \\
\hline Nepean & 822 & 23.9 & 247 & 7.2 & 847 & 24.6 & 1846 & 53.7 & 625 & 18.2 & 268 & 7.8 & 3439 & 100.0 \\
\hline Westmead & 1428 & 33.5 & 317 & 7.4 & 666 & 15.6 & 1913 & 44.8 & 677 & 15.9 & 341 & 8.0 & 4266 & 100.0 \\
\hline The Hills Private & 281 & 21.8 & 27 & 2.1 & 167 & 12.9 & 441 & 34.2 & 55 & 4.3 & 79 & 6.1 & 1290 & 100.0 \\
\hline Hawkesbury & 84 & 9.8 & 55 & 6.4 & 172 & 20.0 & 430 & 50.1 & 167 & 19.4 & 122 & 14.2 & 859 & 100.0 \\
\hline Nepean Private & 104 & 11.0 & 37 & 3.9 & 255 & 27.1 & 536 & 56.9 & 239 & 25.4 & 36 & 3.8 & 942 & 100.0 \\
\hline Westmead Private & 181 & 10.1 & 51 & 2.9 & 253 & 14.2 & 783 & 43.9 & 395 & 22.1 & 77 & 4.3 & 1784 & 100.0 \\
\hline Other Area hospitals & 27 & 11.1 & 9 & 3.7 & 47 & 19.3 & 109 & 44.9 & 38 & 15.6 & 39 & 16.0 & 243 & 100.0 \\
\hline ALL HOSPITALS & 3651 & 21.3 & 978 & 5.7 & 3279 & 19.1 & 8181 & 47.7 & 2699 & 15.8 & 1646 & 9.6 & 17135 & 100.0 \\
\hline \multicolumn{15}{|c|}{ Northern Sydney \& Central Coast } \\
\hline Gosford & 475 & 19.6 & 115 & 4.7 & 725 & 29.9 & 1095 & 45.1 & 529 & 21.8 & 96 & 4.0 & 2426 & 100.0 \\
\hline Wyong & 0 & 0.0 & 5 & 2.2 & 31 & 13.9 & 90 & 40.4 & 3 & 1.3 & 22 & 9.9 & 223 & 100.0 \\
\hline Hornsby & 320 & 35.6 & 37 & 4.1 & 191 & 21.2 & 456 & 50.7 & 86 & 9.6 & 44 & 4.9 & 899 & 100.0 \\
\hline Manly & 157 & 23.8 & 17 & 2.6 & 123 & 18.6 & 276 & 41.8 & 124 & 18.8 & 72 & 10.9 & 661 & 100.0 \\
\hline Mona Vale & 204 & 31.8 & 9 & 1.4 & 255 & 39.7 & 253 & 39.4 & 119 & 18.5 & 59 & 9.2 & 642 & 100.0 \\
\hline Royal North Shore & 760 & 31.7 & 79 & 3.3 & 448 & 18.7 & 1114 & 46.4 & 572 & 23.8 & 124 & 5.2 & 2399 & 100.0 \\
\hline Mater, North Sydney & 504 & 22.7 & 33 & 1.5 & 229 & 10.3 & 766 & 34.5 & 482 & 21.7 & 55 & 2.5 & 2219 & 100.0 \\
\hline North Shore Private & 1276 & 49.9 & 44 & 1.7 & 156 & 6.1 & 728 & 28.5 & 852 & 33.3 & 56 & 2.2 & 2555 & 100.0 \\
\hline Sydney Adventist & 1152 & 52.5 & 70 & 3.2 & 235 & 10.7 & 822 & 37.4 & 326 & 14.9 & 66 & 3.0 & 2195 & 100.0 \\
\hline North Gosford Private & 55 & 6.4 & 24 & 2.8 & 128 & 14.9 & 322 & 37.4 & 297 & 34.5 & 64 & 7.4 & 860 & 100.0 \\
\hline Other Area hospitals & 0 & 0.0 & 0 & 0.0 & 6 & 4.5 & 47 & 35.3 & 1 & 0.8 & 46 & 34.6 & 133 & 100.0 \\
\hline ALL HOSPITALS & 4903 & 32.2 & 433 & 2.8 & 2527 & 16.6 & 5969 & 39.2 & 3391 & 22.3 & 704 & 4.6 & 15212 & 100.0 \\
\hline
\end{tabular}


TABLE 123 (continued)

PAIN RELIEF BY HOSPITAL, NSW 2005"

\begin{tabular}{|c|c|c|c|c|c|c|c|c|c|c|c|c|c|c|}
\hline \multirow[t]{3}{*}{ Health Area and Hospital } & \multirow{2}{*}{\multicolumn{2}{|c|}{ Epidural }} & \multirow{2}{*}{\multicolumn{2}{|c|}{$\begin{array}{c}\text { General } \\
\text { anaesthetic }\end{array}$}} & \multicolumn{4}{|c|}{ Type of pain relief } & \multirow{2}{*}{\multicolumn{2}{|c|}{ Spinal }} & \multirow{2}{*}{\multicolumn{2}{|c|}{ Nil }} & \multirow{2}{*}{\multicolumn{2}{|c|}{ TOTAL }} \\
\hline & & & & & & $\begin{array}{l}\text { M } \\
\text { otics }\end{array}$ & & & & & & & & \\
\hline & No. & $\%$ & No. & $\%$ & No. & $\%$ & No. & $\%$ & No. & $\%$ & No. & $\%$ & No. & $\%$ \\
\hline \multicolumn{15}{|l|}{ Hunter \& New England } \\
\hline Armidale & 17 & 4.2 & 25 & 6.2 & 127 & 31.4 & 212 & 52.3 & 64 & 15.8 & 27 & 6.7 & 405 & 100.0 \\
\hline Inverell & 0 & 0.0 & 3 & 1.2 & 46 & 19.0 & 102 & 42.1 & 73 & 30.2 & 58 & 24.0 & 242 & 100.0 \\
\hline Moree & 10 & 4.2 & 5 & 2.1 & 53 & 22.1 & 108 & 45.0 & 51 & 21.3 & 45 & 18.8 & 240 & 100.0 \\
\hline Tamworth Base & 80 & 10.1 & 81 & 10.2 & 182 & 23.0 & 450 & 56.8 & 98 & 12.4 & 67 & 8.5 & 792 & 100.0 \\
\hline Manning Base & 22 & 3.2 & 41 & 5.9 & 271 & 38.8 & 372 & 53.3 & 122 & 17.5 & 72 & 10.3 & 698 & 100.0 \\
\hline Maitland & 128 & 8.2 & 76 & 4.8 & 245 & 15.6 & 785 & 50.1 & 348 & 22.2 & 221 & 14.1 & 1568 & 100.0 \\
\hline Muswellbrook & 2 & 0.8 & 1 & 0.4 & 57 & 23.7 & 119 & 49.4 & 56 & 23.2 & 53 & 22.0 & 241 & 100.0 \\
\hline Belmont & 19 & 5.9 & 16 & 5.0 & 96 & 29.9 & 147 & 45.8 & 42 & 13.1 & 60 & 18.7 & 321 & 100.0 \\
\hline John Hunter & 670 & 19.8 & 205 & 6.1 & 802 & 23.7 & 1512 & 44.7 & 601 & 17.8 & 420 & 12.4 & 3384 & 100.0 \\
\hline Newcastle Private & 539 & 32.5 & 43 & 2.6 & 255 & 15.4 & 661 & 39.8 & 435 & 26.2 & 101 & 6.1 & 1659 & 100.0 \\
\hline Other Area hospitals & 44 & 5.0 & 47 & 5.4 & 201 & 23.0 & 498 & 57.0 & 104 & 11.9 & 125 & 14.3 & 873 & 100.0 \\
\hline ALL HOSPITALS & 1531 & 14.7 & 543 & 5.2 & 2335 & 22.4 & 4966 & 47.6 & 1994 & 19.1 & 1249 & 12.0 & 10423 & 100.0 \\
\hline \multicolumn{15}{|l|}{ North Coast } \\
\hline Coffs Harbour & 40 & 4.7 & 41 & 4.9 & 136 & 16.1 & 404 & 47.9 & 169 & 20.0 & 171 & 20.3 & 844 & 100.0 \\
\hline Grafton Base & 55 & 12.6 & 35 & 8.0 & 77 & 17.6 & 224 & 51.1 & 72 & 16.4 & 67 & 15.3 & 438 & 100.0 \\
\hline Kempsey & 39 & 12.9 & 8 & 2.6 & 111 & 36.6 & 159 & 52.5 & 16 & 5.3 & 55 & 18.2 & 303 & 100.0 \\
\hline Lismore Base & 126 & 10.0 & 44 & 3.5 & 212 & 16.9 & 576 & 45.9 & 245 & 19.5 & 122 & 9.7 & 1256 & 100.0 \\
\hline Murwillumbah & 25 & 6.2 & 15 & 3.7 & 131 & 32.5 & 180 & 44.7 & 111 & 27.5 & 46 & 11.4 & 403 & 100.0 \\
\hline Tweed Heads & 51 & 4.9 & 34 & 3.3 & 284 & 27.2 & 558 & 53.3 & 201 & 19.2 & 140 & 13.4 & 1046 & 100.0 \\
\hline Port Macquarie Base & 49 & 6.5 & 26 & 3.5 & 207 & 27.5 & 388 & 51.6 & 147 & 19.5 & 115 & 15.3 & 752 & 100.0 \\
\hline Other Area hospitals & 10 & 2.3 & 1 & 0.2 & 64 & 14.8 & 178 & 41.1 & 13 & 3.0 & 178 & 41.1 & 433 & 100.0 \\
\hline ALL HOSPITALS & 395 & 7.2 & 204 & 3.7 & 1222 & 22.3 & 2667 & 48.7 & 974 & 17.8 & 894 & 16.3 & 5475 & 100.0 \\
\hline \multicolumn{15}{|l|}{ Greater Southern } \\
\hline Goulburn Base & 49 & 17.1 & 20 & 7.0 & 51 & 17.8 & 172 & 60.1 & 18 & 6.3 & 22 & 7.7 & 286 & 100.0 \\
\hline Moruya & 17 & 5.2 & 23 & 7.0 & 79 & 23.9 & 150 & 45.5 & 51 & 15.5 & 76 & 23.0 & 330 & 100.0 \\
\hline Queanbeyan & 31 & 11.2 & 14 & 5.1 & 65 & 23.5 & 141 & 50.9 & 19 & 6.9 & 70 & 25.3 & 277 & 100.0 \\
\hline Griffith Base & 14 & 3.2 & 6 & 1.4 & 199 & 44.8 & 228 & 51.4 & 107 & 24.1 & 51 & 11.5 & 444 & 100.0 \\
\hline Wagga Wagga Base & 43 & 6.1 & 19 & 2.7 & 197 & 27.8 & 372 & 52.5 & 140 & 19.8 & 115 & 16.2 & 708 & 100.0 \\
\hline Calvary, Wagga Wagga & 22 & 4.0 & 14 & 2.5 & 141 & 25.4 & 218 & 39.2 & 174 & 31.3 & 54 & 9.7 & 556 & 100.0 \\
\hline Other Area hospitals & 70 & 5.1 & 32 & 2.3 & 384 & 28.1 & 742 & 54.4 & 255 & 18.7 & 243 & 17.8 & 1365 & 100.0 \\
\hline ALL HOSPITALS & 246 & 6.2 & 128 & 3.2 & 1116 & 28.1 & 2023 & 51.0 & 764 & 19.3 & 631 & 15.9 & 3966 & 100.0 \\
\hline \multicolumn{15}{|l|}{ Greater Western } \\
\hline Dubbo Base & 80 & 6.7 & 96 & 8.0 & 349 & 29.1 & 672 & 56.0 & 115 & 9.6 & 157 & 13.1 & 1200 & 100.0 \\
\hline Mudgee & 3 & 1.3 & 18 & 7.8 & 62 & 26.7 & 133 & 57.3 & 42 & 18.1 & 36 & 15.5 & 232 & 100.0 \\
\hline Bathurst Base & 103 & 17.7 & 31 & 5.3 & 34 & 5.8 & 273 & 46.9 & 83 & 14.3 & 88 & 15.1 & 582 & 100.0 \\
\hline Orange Base & 74 & 9.4 & 68 & 8.6 & 149 & 18.9 & 421 & 53.5 & 117 & 14.9 & 119 & 15.1 & 787 & 100.0 \\
\hline Broken Hill Base & 4 & 1.5 & 9 & 3.3 & 59 & 21.9 & 145 & 53.7 & 54 & 20.0 & 50 & 18.5 & 270 & 100.0 \\
\hline Other Area hospitals & 32 & 5.0 & 25 & 3.9 & 131 & 20.5 & 285 & 44.5 & 121 & 18.9 & 157 & 24.5 & 640 & 100.0 \\
\hline ALL HOSPITALS & 296 & 8.0 & 247 & 6.7 & 784 & 21.1 & 1929 & 52.0 & 532 & 14.3 & 607 & 16.4 & 3711 & 100.0 \\
\hline TOTAL & 19278 & 21.6 & 3997 & 4.5 & 17159 & 19.3 & 40087 & 45.0 & 15094 & 17.0 & 9161 & 10.3 & 89140 & 100.0 \\
\hline
\end{tabular}




\section{Perineal status in selected hospitals}

Table 124 show the perineal status in vaginal births for individual hospitals where the number of reported confinements exceeded 200 in 2005, totals for all hospitals within each health area and the NSW total.
In addition to the perinatal outcomes described in the table there were a total of 96 cases of fourth degree tear reported in 2005 .

\section{TABLE 124}

\section{VAGINAL BIRTHS BY PERINEAL STATUS AND HOSPITAL, NSW 2005}

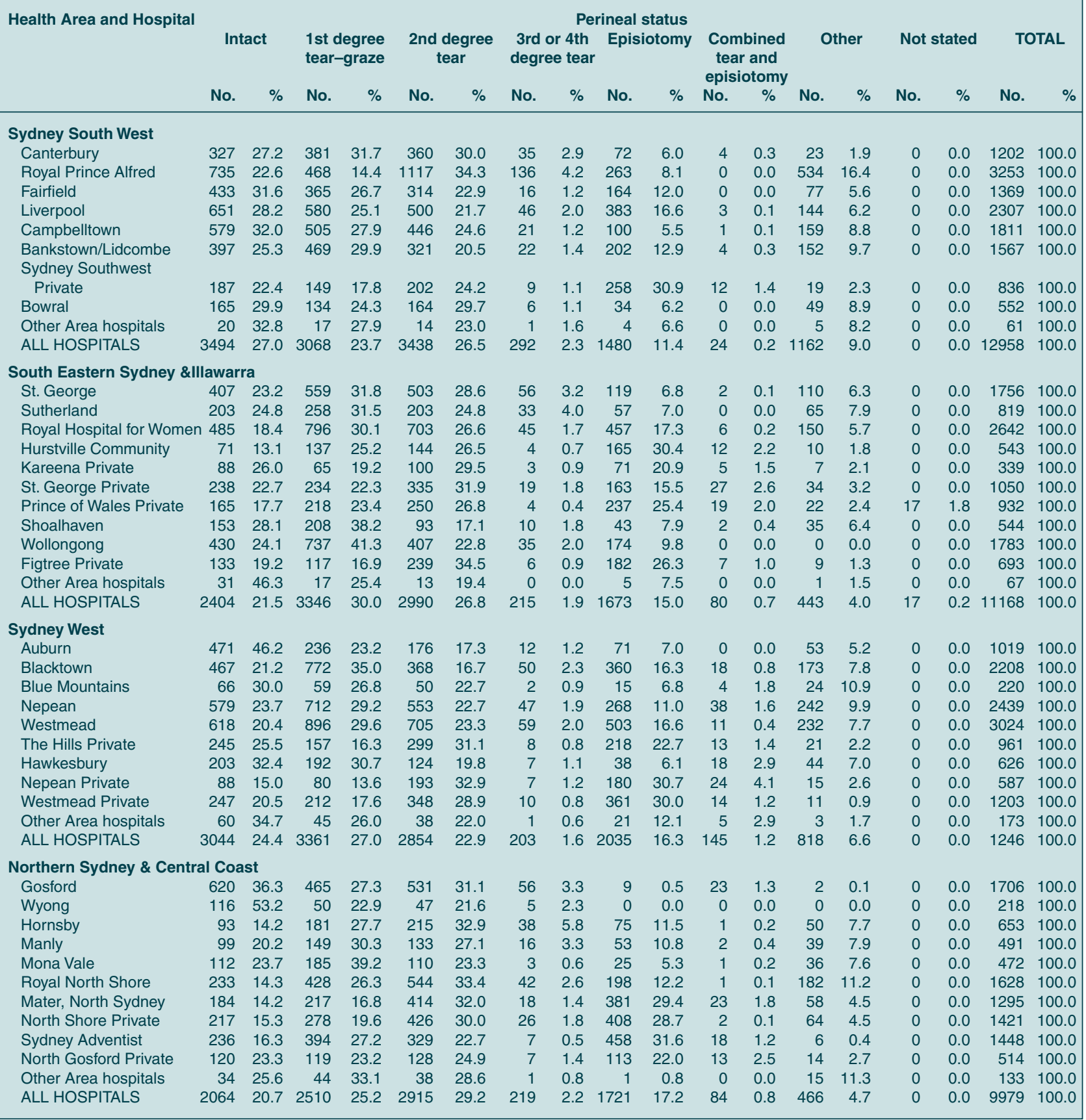


TABLE 124 (continued)

VAGINAL BIRTHS BY PERINEAL STATUS AND HOSPITAL, NSW 2005"

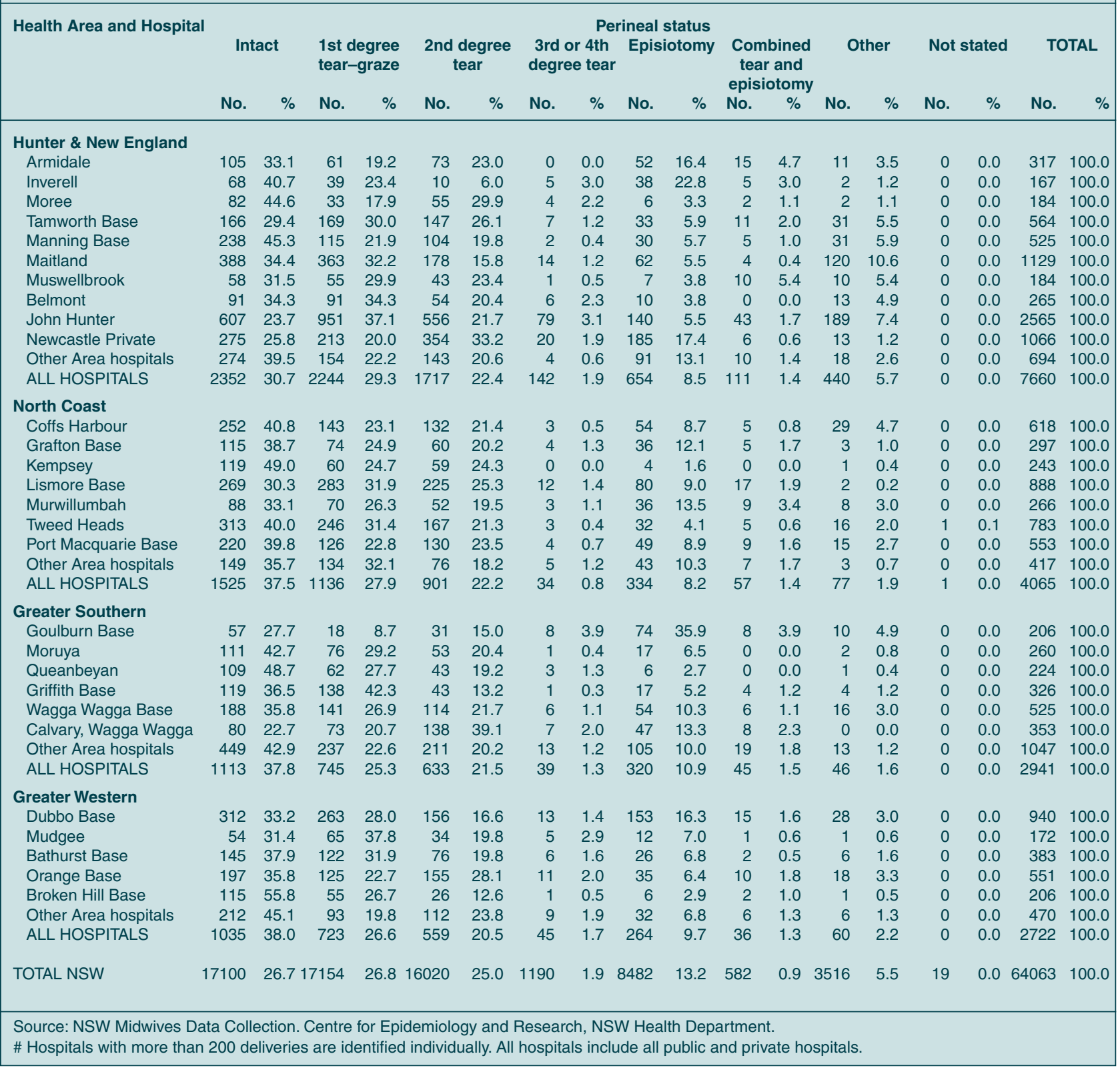




\section{Birth weight in selected hospitals}

Table 125 shows the birth weight of babies for individual hospitals where the number of reported confinements exceeded 200 in 2005, totals for all hospitals within each health area and the NSW total.

\section{TABLE 125}

BIRTHS BY BABY BIRTH WEIGHT AND HOSPITAL, NSW 2005"

\begin{tabular}{|c|c|c|c|c|c|c|c|c|c|c|c|c|}
\hline \multirow[t]{3}{*}{ Health Area and Hospital } & \multicolumn{12}{|c|}{ Birth weight (grams) } \\
\hline & \multicolumn{2}{|c|}{$\begin{array}{c}\text { Less than } \\
1,000\end{array}$} & \multicolumn{2}{|c|}{$\begin{array}{c}1,000- \\
1,499\end{array}$} & \multicolumn{2}{|c|}{$\begin{array}{l}1,500- \\
2,499\end{array}$} & \multicolumn{2}{|c|}{$2,500+$} & \multicolumn{2}{|c|}{ Not stated } & \multicolumn{2}{|c|}{ TOTAL } \\
\hline & No. & $\%$ & No. & $\%$ & No. & $\%$ & No. & $\%$ & No. & $\%$ & No. & $\%$ \\
\hline \multicolumn{13}{|l|}{ Sydney South West } \\
\hline Fairfield & 7 & 0.4 & 1 & 0.1 & 59 & 3.5 & 1633 & 96.1 & 0 & 0.0 & 1700 & 100.0 \\
\hline Bankstown-Lidcombe & 9 & 0.5 & 3 & 0.2 & 78 & 4.1 & 1818 & 95.2 & 2 & 0.1 & 1910 & 100.0 \\
\hline Liverpool & 52 & 1.7 & 67 & 2.2 & 254 & 8.3 & 2697 & 87.7 & 5 & 0.2 & 3075 & 100.0 \\
\hline Campbelltown & 9 & 0.4 & 1 & 0.0 & 106 & 4.6 & 2206 & 94.8 & 6 & 0.3 & 2328 & 100.0 \\
\hline Royal Prince Alfred & 59 & 1.3 & 54 & 1.2 & 286 & 6.2 & 4230 & 91.3 & 2 & 0.0 & 4631 & 100.0 \\
\hline Canterbury & 4 & 0.3 & 0 & 0.0 & 56 & 3.6 & 1503 & 96.2 & 0 & 0.0 & 1563 & 100.0 \\
\hline Sydney Southwest Private & 3 & 0.3 & 0 & 0.0 & 37 & 3.1 & 1148 & 96.6 & 0 & 0.0 & 1188 & 100.0 \\
\hline Bowral & 3 & 0.4 & 1 & 0.1 & 28 & 3.9 & 678 & 95.4 & 1 & 0.1 & 711 & 00.0 \\
\hline Other Area hospitals & 0 & 0.0 & 0 & 0.0 & 2 & 2.9 & 66 & 97.1 & 0 & 0.0 & 68 & 100.0 \\
\hline ALL HOSPITALS & 146 & 0.9 & 127 & 0.7 & 906 & 5.3 & 15979 & 93.0 & 16 & 0.1 & 17174 & 100.0 \\
\hline \multicolumn{13}{|c|}{ South Eastern Sydney \& Illawarra } \\
\hline St. George & 12 & 0.5 & 6 & 0.3 & 124 & 5.3 & 2198 & 93.9 & 2 & 0.1 & 2342 & 100.0 \\
\hline St. George Private & 5 & 0.3 & 1 & 0.1 & 89 & 5.0 & 1698 & 94.6 & 1 & 0.1 & 1794 & 100.0 \\
\hline Royal Hospital for Women & 64 & 1.6 & 40 & 1.0 & 246 & 6.2 & 3581 & 91.0 & 6 & 0.2 & 3937 & 100.0 \\
\hline Wollongong & 15 & 0.7 & 8 & 0.3 & 145 & 6.3 & 2134 & 92.6 & 3 & 0.1 & 2305 & 100.0 \\
\hline Hurstville Community & 1 & 0.1 & 3 & 0.3 & 30 & 3.3 & 872 & 96.2 & 0 & 0.0 & 906 & 100.0 \\
\hline Sutherland & 3 & 0.3 & 0 & 0.0 & 30 & 2.8 & 1021 & 96.8 & 1 & 0.1 & 1055 & 100.0 \\
\hline Shoalhaven & 7 & 0.9 & 3 & 0.4 & 43 & 5.3 & 763 & 93.3 & 2 & 0.2 & 818 & 100.0 \\
\hline Kareena Private & 1 & 0.2 & 2 & 0.3 & 26 & 4.1 & 610 & 95.5 & 0 & 0.0 & 639 & 100.0 \\
\hline Prince of Wales Private & 1 & 0.1 & 1 & 0.1 & 39 & 2.4 & 1565 & 97.3 & 3 & 0.2 & 1609 & 100.0 \\
\hline Figtree Private & 0 & 0.0 & 0 & 0.0 & 15 & 1.5 & 973 & 98.5 & 0 & 0.0 & 988 & 100.0 \\
\hline Other Area hospitals & 0 & 0.0 & 0 & 0.0 & 3 & 3.5 & 81 & 94.2 & 2 & 2.3 & 86 & 100.0 \\
\hline ALL HOSPITALS & 109 & 0.7 & 64 & 0.4 & 790 & 4.8 & 15496 & 94.0 & 20 & 0.1 & 16479 & 100.0 \\
\hline \multicolumn{13}{|l|}{ Sydney West } \\
\hline Nepean & 44 & 1.3 & 34 & 1.0 & 257 & 7.3 & 3172 & 90.4 & 0 & 0.0 & 3507 & 100.0 \\
\hline Blacktown & 9 & 0.3 & 7 & 0.2 & 138 & 4.8 & 2710 & 94.6 & 0 & 0.0 & 2864 & 100.0 \\
\hline Westmead & 64 & 1.5 & 84 & 1.9 & 358 & 8.1 & 3901 & 88.5 & 1 & 0.0 & 4408 & 100.0 \\
\hline The Hills Private & 2 & 0.2 & 2 & 0.2 & 47 & 3.6 & 1266 & 96.1 & 0 & 0.0 & 1317 & 100.0 \\
\hline Nepean Private & 3 & 0.3 & 0 & 0.0 & 32 & 3.3 & 924 & 96.3 & 1 & 0.1 & 960 & 100.0 \\
\hline Blue Mountains & 1 & 0.4 & 0 & 0.0 & 3 & 1.1 & 265 & 98.5 & 0 & 0.0 & 269 & 100.0 \\
\hline Westmead Private & 3 & 0.2 & 2 & 0.1 & 54 & 3.0 & 1745 & 96.7 & 0 & 0.0 & 1804 & 100.0 \\
\hline Hawkesbury & 2 & 0.2 & 2 & 0.2 & 28 & 3.2 & 833 & 96.3 & 0 & 0.0 & 865 & 100.0 \\
\hline Auburn & 4 & 0.3 & 1 & 0.1 & 33 & 2.7 & 1171 & 96.7 & 2 & 0.2 & 1211 & 100.0 \\
\hline Other Area hospitals & 2 & 0.8 & 1 & 0.4 & 13 & 5.3 & 229 & 93.5 & 0 & 0.0 & 245 & 100.0 \\
\hline ALL HOSPITALS & 134 & 0.8 & 133 & 0.8 & 963 & 5.5 & 16216 & 92.9 & 4 & 0.0 & 17450 & 100.0 \\
\hline \multicolumn{13}{|c|}{ Northern Sydney \& Central Coast } \\
\hline Manly & 3 & 0.5 & 0 & 0.0 & 20 & 3.0 & 639 & 96.4 & 1 & 0.2 & 663 & 100.0 \\
\hline Gosford & 9 & 0.4 & 3 & 0.1 & 119 & 4.9 & 2315 & 94.6 & 2 & 0.1 & 2448 & 100.0 \\
\hline Sydney Adventist & 3 & 0.1 & 1 & 0.0 & 65 & 2.9 & 2156 & 96.9 & 1 & 0.0 & 2226 & 100.0 \\
\hline North Shore Private & 13 & 0.5 & 5 & 0.2 & 96 & 3.7 & 2490 & 95.6 & 0 & 0.0 & 2604 & 100.0 \\
\hline Royal North Shore & 37 & 1.5 & 63 & 2.5 & 186 & 7.5 & 2187 & 88.4 & 2 & 0.1 & 2475 & 100.0 \\
\hline Hornsby & 2 & 0.2 & 5 & 0.5 & 33 & 3.6 & 875 & 95.6 & 0 & 0.0 & 915 & 100.0 \\
\hline Mona Vale & 1 & 0.2 & 0 & 0.0 & 15 & 2.3 & 633 & 97.5 & 0 & 0.0 & 649 & 100.0 \\
\hline Mater, North Sydney & 2 & 0.1 & 2 & 0.1 & 99 & 4.4 & 2163 & 95.5 & 0 & 0.0 & 2266 & 100.0 \\
\hline North Gosford Private & 0 & 0.0 & 2 & 0.2 & 45 & 5.1 & 830 & 94.6 & 0 & 0.0 & 877 & 00.0 \\
\hline Wyong & 0 & 0.0 & 0 & 0.0 & 3 & 1.3 & 220 & 98.7 & 0 & 0.0 & 223 & 100.0 \\
\hline Other Area hospitals & 0 & 0.0 & 0 & 0.0 & 1 & 0.8 & 132 & 99.2 & 0 & 0.0 & 133 & 100.0 \\
\hline ALL HOSPITALS & 70 & 0.5 & 81 & 0.5 & 682 & 4.4 & 14640 & 94.6 & 6 & 0.0 & 15479 & 100.0 \\
\hline
\end{tabular}


TABLE 125 (continued)

BIRTHS BY BABY BIRTH WEIGHT AND HOSPITAL, NSW 2005"

\begin{tabular}{|c|c|c|c|c|c|c|c|c|c|c|c|c|}
\hline \multirow[t]{3}{*}{ Health Area and Hospital } & \multicolumn{12}{|c|}{ Birth weight (grams) } \\
\hline & \multicolumn{2}{|c|}{$\begin{array}{c}\text { Less than } \\
1,000\end{array}$} & \multicolumn{2}{|c|}{$\begin{array}{c}1,000- \\
1,499\end{array}$} & \multicolumn{2}{|c|}{$\begin{array}{l}1,500- \\
2,499\end{array}$} & \multicolumn{2}{|c|}{$2,500+$} & \multicolumn{2}{|c|}{ Not stated } & \multicolumn{2}{|c|}{ TOTAL } \\
\hline & No. & $\%$ & No. & $\%$ & No. & $\%$ & No. & $\%$ & No. & $\%$ & No. & $\%$ \\
\hline \multicolumn{13}{|l|}{ Hunter \& New England } \\
\hline John Hunter & 83 & 2.4 & 63 & 1.8 & 291 & 8.4 & 3037 & 87.4 & 1 & 0.0 & 3475 & 100.0 \\
\hline Newcastle Private & 12 & 0.7 & 3 & 0.2 & 63 & 3.7 & 1614 & 95.2 & 4 & 0.2 & 1696 & 100.0 \\
\hline Maitland & 8 & 0.5 & 2 & 0.1 & 81 & 5.1 & 1498 & 94.3 & 0 & 0.0 & 1589 & 100.0 \\
\hline Tamworth Base & 5 & 0.6 & 4 & 0.5 & 49 & 6.1 & 741 & 92.6 & 1 & 0.1 & 800 & 100.0 \\
\hline Muswellbrook & 1 & 0.4 & 0 & 0.0 & 7 & 2.9 & 237 & 96.7 & 0 & 0.0 & 245 & 100.0 \\
\hline Armidale & 2 & 0.5 & 0 & 0.0 & 34 & 8.3 & 374 & 91.0 & 1 & 0.2 & 411 & 100.0 \\
\hline Moree & 1 & 0.4 & 1 & 0.4 & 11 & 4.6 & 228 & 94.6 & 0 & 0.0 & 241 & 100.0 \\
\hline Manning Base & 3 & 0.4 & 0 & 0.0 & 23 & 3.3 & 678 & 96.3 & 0 & 0.0 & 704 & 100.0 \\
\hline Inverell & 0 & 0.0 & 0 & 0.0 & 21 & 8.4 & 229 & 91.2 & 1 & 0.4 & 251 & 100.0 \\
\hline Belmont & 0 & 0.0 & 0 & 0.0 & 9 & 2.8 & 313 & 97.2 & 0 & 0.0 & 322 & 100.0 \\
\hline Other Area hospitals & 2 & 0.2 & 0 & 0.0 & 27 & 3.1 & 847 & 96.6 & 1 & 0.1 & 877 & 100.0 \\
\hline ALL HOSPITALS & 117 & 1.1 & 73 & 0.7 & 616 & 5.8 & 9796 & 92.3 & 9 & 0.1 & 10611 & 100.0 \\
\hline \multicolumn{13}{|l|}{ North Coast } \\
\hline Tweed Heads & 4 & 0.4 & 5 & 0.5 & 41 & 3.9 & 1006 & 95.3 & 0 & 0.0 & 1056 & 100.0 \\
\hline Lismore Base & 7 & 0.5 & 7 & 0.5 & 62 & 4.8 & 1202 & 94.0 & 1 & 0.1 & 1279 & 100.0 \\
\hline Kempsey & 3 & 1.0 & 0 & 0.0 & 9 & 3.0 & 291 & 96.0 & 0 & 0.0 & 303 & 100.0 \\
\hline Grafton Base & 1 & 0.2 & 1 & 0.2 & 16 & 3.6 & 421 & 95.7 & 1 & 0.2 & 440 & 100.0 \\
\hline Port Macquarie Base & 1 & 0.1 & 1 & 0.1 & 33 & 4.3 & 727 & 95.4 & 0 & 0.0 & 762 & 100.0 \\
\hline Coffs Harbour & 0 & 0.0 & 7 & 0.8 & 40 & 4.7 & 805 & 94.5 & 0 & 0.0 & 852 & 100.0 \\
\hline Murwillumbah & 0 & 0.0 & 2 & 0.5 & 15 & 3.7 & 390 & 95.8 & 0 & 0.0 & 407 & 100.0 \\
\hline Other Area hospitals & 1 & 0.2 & 0 & 0.0 & 5 & 1.2 & 424 & 97.9 & 3 & 0.7 & 433 & 100.0 \\
\hline ALL HOSPITALS & 17 & 0.3 & 23 & 0.4 & 221 & 4.0 & 5266 & 95.2 & 5 & 0.1 & 5532 & 100.0 \\
\hline \multicolumn{13}{|l|}{ Greater Southern } \\
\hline Goulburn Base & 2 & 0.7 & 0 & 0.0 & 18 & 6.2 & 272 & 93.2 & 0 & 0.0 & 292 & 100.0 \\
\hline Griffith Base & 2 & 0.4 & 0 & 0.0 & 13 & 2.9 & 434 & 96.7 & 0 & 0.0 & 449 & 100.0 \\
\hline Wagga Wagga Base & 3 & 0.4 & 3 & 0.4 & 37 & 5.1 & 679 & 94.0 & 0 & 0.0 & 722 & 100.0 \\
\hline Calvary, Wagga Wagga & 0 & 0.0 & 1 & 0.2 & 20 & 3.5 & 549 & 96.3 & 0 & 0.0 & 570 & 100.0 \\
\hline Moruya & 0 & 0.0 & 0 & 0.0 & 22 & 6.6 & 313 & 93.4 & 0 & 0.0 & 335 & 100.0 \\
\hline Queanbeyan & 0 & 0.0 & 0 & 0.0 & 5 & 1.8 & 272 & 98.2 & 0 & 0.0 & 277 & 100.0 \\
\hline Other Area hospitals & 3 & 0.2 & 2 & 0.1 & 36 & 2.6 & 1327 & 97.0 & 0 & 0.0 & 1368 & 100.0 \\
\hline ALL HOSPITALS & 10 & 0.2 & 6 & 0.1 & 151 & 3.8 & 3846 & 95.8 & 0 & 0.0 & 4013 & 100.0 \\
\hline \multicolumn{13}{|l|}{ Greater Western } \\
\hline Bathurst Base & 2 & 0.3 & 0 & 0.0 & 39 & 6.6 & 552 & 93.1 & 0 & 0.0 & 593 & 100.0 \\
\hline Dubbo Base & 10 & 0.8 & 1 & 0.1 & 87 & 7.1 & 1119 & 91.7 & 3 & 0.2 & 1220 & 100.0 \\
\hline Broken Hill Base & 1 & 0.4 & 0 & 0.0 & 11 & 4.1 & 259 & 95.6 & 0 & 0.0 & 271 & 100.0 \\
\hline Orange Base & 1 & 0.1 & 2 & 0.3 & 49 & 6.1 & 747 & 93.4 & 1 & 0.1 & 800 & 100.0 \\
\hline Mudgee & 0 & 0.0 & 0 & 0.0 & 4 & 1.7 & 228 & 98.3 & 0 & 0.0 & 232 & 100.0 \\
\hline Other Area hospitals & 2 & 0.3 & 3 & 0.5 & 31 & 4.8 & 608 & 94.4 & 0 & 0.0 & 644 & 100.0 \\
\hline ALL HOSPITALS & 16 & 0.4 & 6 & 0.2 & 221 & 5.9 & 3513 & 93.4 & 4 & 0.1 & 3760 & 100.0 \\
\hline TOTAL NSW & 619 & 0.7 & 513 & 0.6 & 4552 & 5.0 & 84859 & 93.7 & 67 & 0.1 & 90610 & 100.0 \\
\hline
\end{tabular}




\section{Gestational age in selected hospitals}

Table 126 shows the gestational age of babies for individual hospitals where the number of reported confinements exceeded 200 in 2005, totals for all hospitals within each health area, and the NSW total.

\begin{tabular}{|c|c|c|c|c|c|c|c|c|c|c|c|c|}
\hline BIRTHS BY GESTATIONA & E AN & SPI & NS & & & & & & & & & \\
\hline Health Area and Hospital & & & & & & estati & onal age & eeks) & & & & \\
\hline & & & & & & & & & Not & ted & & IAL \\
\hline & No. & $\%$ & No. & $\%$ & No. & $\%$ & No. & $\%$ & No. & $\%$ & No. & $\%$ \\
\hline Sydney South West & & & & & & & & & & & & \\
\hline Canterbury & 6 & 0.4 & 3 & 0.2 & 53 & 3.4 & 1501 & 96.0 & 0 & 0.0 & 1563 & 100.0 \\
\hline Royal Prince Alfred & 143 & 3.1 & 81 & 1.7 & 245 & 5.3 & 4162 & 89.9 & 0 & 0.0 & 4631 & 100.0 \\
\hline Fairfield & 11 & 0.6 & 2 & 0.1 & 54 & 3.2 & 1633 & 96.1 & 0 & 0.0 & 1700 & 100.0 \\
\hline Campbelltown & 12 & 0.5 & 13 & 0.6 & 96 & 4.1 & 2207 & 94.8 & 0 & 0.0 & 2328 & 100.0 \\
\hline Bankstown-Lidcombe & 12 & 0.6 & 5 & 0.3 & 66 & 3.5 & 1827 & 95.7 & 0 & 0.0 & 1910 & 100.0 \\
\hline Liverpool & 127 & 4.1 & 93 & 3.0 & 165 & 5.4 & 2690 & 87.5 & 0 & 0.0 & 3075 & 100.0 \\
\hline Sydney Southwest Private & 3 & 0.3 & 2 & 0.2 & 57 & 4.8 & 1126 & 94.8 & 0 & 0.0 & 1188 & 100.0 \\
\hline Bowral & 5 & 0.7 & 2 & 0.3 & 20 & 2.8 & 684 & 96.2 & 0 & 0.0 & 711 & 100.0 \\
\hline Other Area hospitals & 0 & 0.0 & 0 & 0.0 & 2 & 2.9 & 66 & 97.1 & 0 & 0.0 & 68 & 100.0 \\
\hline ALL HOSPITALS & 319 & 1.9 & 201 & 1.2 & 758 & 4.4 & 15896 & 92.6 & 0 & 0.0 & 17174 & 100.0 \\
\hline South Eastern Sydney \&IIla & & & & & & & & & & & & \\
\hline St. George & 16 & 0.7 & 18 & 0.8 & 121 & 5.2 & 2186 & 93.3 & 1 & 0.0 & 2342 & 100.0 \\
\hline Royal Hospital for Women & 112 & 2.8 & 78 & 2.0 & 206 & 5.2 & 3541 & 89.9 & 0 & 0.0 & 3937 & 100.0 \\
\hline Hurstville Community & 2 & 0.2 & 3 & 0.3 & 40 & 4.4 & 861 & 95.0 & 0 & 0.0 & 906 & 100.0 \\
\hline St. George Private & 5 & 0.3 & 3 & 0.2 & 96 & 5.4 & 1690 & 94.2 & 0 & 0.0 & 1794 & 100.0 \\
\hline Wollongong & 25 & 1.1 & 29 & 1.3 & 171 & 7.4 & 2079 & 90.2 & 1 & 0.0 & 2305 & 100.0 \\
\hline Kareena Private & 2 & 0.3 & 2 & 0.3 & 45 & 7.0 & 590 & 92.3 & 0 & 0.0 & 639 & 100.0 \\
\hline Shoalhaven & 13 & 1.6 & 7 & 0.9 & 40 & 4.9 & 758 & 92.7 & 0 & 0.0 & 818 & 100.0 \\
\hline Sutherland & 4 & 0.4 & 3 & 0.3 & 36 & 3.4 & 1012 & 95.9 & 0 & 0.0 & 1055 & 100.0 \\
\hline Prince of Wales Private & 2 & 0.1 & 1 & 0.1 & 64 & 4.0 & 1537 & 95.5 & 5 & 0.3 & 1609 & 100.0 \\
\hline Figtree Private & 0 & 0.0 & 1 & 0.1 & 30 & 3.0 & 957 & 96.9 & 0 & 0.0 & 988 & 100.0 \\
\hline Other Area hospitals & 0 & 0.0 & 0 & 0.0 & 3 & 3.5 & 83 & 96.5 & 0 & 0.0 & 86 & 100.0 \\
\hline ALL HOSPITALS & 181 & 1.1 & 145 & 0.9 & 852 & 5.2 & 15294 & 92.8 & 7 & 0.0 & 16479 & 100.0 \\
\hline Sydney West & & & & & & & & & & & & \\
\hline Blacktown & 14 & 0.5 & 13 & 0.5 & 129 & 4.5 & 2708 & 94.6 & 0 & 0.0 & 2864 & 100.0 \\
\hline Nepean & 89 & 2.5 & 64 & 1.8 & 257 & 7.3 & 3097 & 88.3 & 0 & 0.0 & 3507 & 100.0 \\
\hline Westmead & 146 & 3.3 & 117 & 2.7 & 221 & 5.0 & 3924 & 89.0 & 0 & 0.0 & 4408 & 100.0 \\
\hline Westmead Private & 4 & 0.2 & 5 & 0.3 & 74 & 4.1 & 1721 & 95.4 & 0 & 0.0 & 1804 & 100.0 \\
\hline Auburn & 6 & 0.5 & 2 & 0.2 & 26 & 2.1 & 1177 & 97.2 & 0 & 0.0 & 1211 & 100.0 \\
\hline The Hills Private & 7 & 0.5 & 3 & 0.2 & 61 & 4.6 & 1246 & 94.6 & 0 & 0.0 & 1317 & 100.0 \\
\hline Hawkesbury & 5 & 0.6 & 1 & 0.1 & 37 & 4.3 & 820 & 94.8 & 2 & 0.2 & 865 & 100.0 \\
\hline Nepean Private & 3 & 0.3 & 0 & 0.0 & 54 & 5.6 & 903 & 94.1 & 0 & 0.0 & 960 & 100.0 \\
\hline Blue Mountains & 1 & 0.4 & 0 & 0.0 & 6 & 2.2 & 262 & 97.4 & 0 & 0.0 & 269 & 100.0 \\
\hline Other Area hospitals & 3 & 1.2 & 0 & 0.0 & 12 & 4.9 & 230 & 93.9 & 0 & 0.0 & 245 & 100.0 \\
\hline ALL HOSPITALS & 278 & 1.6 & 205 & 1.2 & 877 & 5.0 & 16088 & 92.2 & 2 & 0.0 & 17450 & 100.0 \\
\hline Northern Sydney \& Central & & & & & & & & & & & & \\
\hline Gosford & 18 & 0.7 & 18 & 0.7 & 146 & 6.0 & 2266 & 92.6 & 0 & 0.0 & 2448 & 100.0 \\
\hline Royal North Shore & 116 & 4.7 & 55 & 2.2 & 120 & 4.8 & 2184 & 88.2 & 0 & 0.0 & 2475 & 100.0 \\
\hline North Shore Private & 15 & 0.6 & 6 & 0.2 & 101 & 3.9 & 2482 & 95.3 & 0 & 0.0 & 2604 & 100.0 \\
\hline Sydney Adventist & 9 & 0.4 & 4 & 0.2 & 107 & 4.8 & 2106 & 94.6 & 0 & 0.0 & 2226 & 100.0 \\
\hline Manly & 4 & 0.6 & 0 & 0.0 & 22 & 3.3 & 637 & 96.1 & 0 & 0.0 & 663 & 100.0 \\
\hline Hornsby & 7 & 0.8 & 0 & 0.0 & 38 & 4.2 & 870 & 95.1 & 0 & 0.0 & 915 & 100.0 \\
\hline Mona Vale & 1 & 0.2 & 1 & 0.2 & 16 & 2.5 & 631 & 97.2 & 0 & 0.0 & 649 & 100.0 \\
\hline Mater, North Sydney & 5 & 0.2 & 17 & 0.8 & 98 & 4.3 & 2146 & 94.7 & 0 & 0.0 & 2266 & 100.0 \\
\hline North Gosford Private & 1 & 0.1 & 3 & 0.3 & 61 & 7.0 & 812 & 92.6 & 0 & 0.0 & 877 & 100.0 \\
\hline Wyong & 0 & 0.0 & 0 & 0.0 & 6 & 2.7 & 217 & 97.3 & 0 & 0.0 & 223 & 100.0 \\
\hline Other Area hospitals & 0 & 0.0 & 0 & 0.0 & 1 & 0.8 & 132 & 99.2 & 0 & 0.0 & 133 & 100.0 \\
\hline ALL HOSPITALS & 176 & 1.1 & 104 & 0.7 & 716 & 4.6 & 14483 & 93.6 & 0 & 0.0 & 15479 & 100.0 \\
\hline
\end{tabular}




\section{TABLE 126 (continued)}

BIRTHS BY GESTATIONAL AGE AND HOSPITAL, NSW $2005^{*}$

\begin{tabular}{|c|c|c|c|c|c|c|c|c|c|c|c|c|}
\hline \multirow[t]{3}{*}{ Health Area and Hospital } & \multicolumn{12}{|c|}{ Gestational age (weeks) } \\
\hline & \multicolumn{2}{|c|}{$<31$} & \multicolumn{2}{|c|}{$32-33$} & \multicolumn{2}{|c|}{$34-36$} & \multicolumn{2}{|c|}{$37+$} & \multicolumn{2}{|c|}{ Not stated } & \multicolumn{2}{|c|}{ TOTAL } \\
\hline & No. & $\%$ & No. & $\%$ & No. & $\%$ & No. & $\%$ & No. & $\%$ & No. & $\%$ \\
\hline \multicolumn{13}{|l|}{ Hunter \& New England } \\
\hline Tamworth Base & 11 & 1.4 & 3 & 0.4 & 51 & 6.4 & 735 & 91.9 & 0 & 0.0 & 800 & 100.0 \\
\hline John Hunter & 170 & 4.9 & 94 & 2.7 & 235 & 6.8 & 2976 & 85.6 & 0 & 0.0 & 3475 & 100.0 \\
\hline Maitland & 12 & 0.8 & 2 & 0.1 & 92 & 5.8 & 1483 & 93.3 & 0 & 0.0 & 1589 & 100.0 \\
\hline Muswellbrook & 1 & 0.4 & 0 & 0.0 & 14 & 5.7 & 230 & 93.9 & 0 & 0.0 & 245 & 100.0 \\
\hline Newcastle Private & 17 & 1.0 & 5 & 0.3 & 104 & 6.1 & 1570 & 92.6 & 0 & 0.0 & 1696 & 100.0 \\
\hline Armidale & 4 & 1.0 & 1 & 0.2 & 25 & 6.1 & 381 & 92.7 & 0 & 0.0 & 411 & 100.0 \\
\hline Moree & 1 & 0.4 & 0 & 0.0 & 10 & 4.1 & 230 & 95.4 & 0 & 0.0 & 241 & 100.0 \\
\hline Manning Base & 4 & 0.6 & 0 & 0.0 & 32 & 4.5 & 668 & 94.9 & 0 & 0.0 & 704 & 100.0 \\
\hline Inverell & 1 & 0.4 & 0 & 0.0 & 15 & 6.0 & 235 & 93.6 & 0 & 0.0 & 251 & 100.0 \\
\hline Belmont & 0 & 0.0 & 1 & 0.3 & 5 & 1.6 & 316 & 98.1 & 0 & 0.0 & 322 & 100.0 \\
\hline Other Area hospitals & 2 & 0.2 & 1 & 0.1 & 11 & 1.3 & 863 & 98.4 & 0 & 0.0 & 877 & 100.0 \\
\hline ALL HOSPITALS & 223 & 2.1 & 107 & 1.0 & 594 & 5.6 & 9687 & 91.3 & 0 & 0.0 & 10611 & 100.0 \\
\hline \multicolumn{13}{|l|}{ North Coast } \\
\hline Lismore Base & 16 & 1.3 & 7 & 0.5 & 81 & 6.3 & 1175 & 91.9 & 0 & 0.0 & 1279 & 100.0 \\
\hline Tweed Heads & 10 & 0.9 & 2 & 0.2 & 42 & 4.0 & 1002 & 94.9 & 0 & 0.0 & 1056 & 100.0 \\
\hline Kempsey & 3 & 1.0 & 0 & 0.0 & 11 & 3.6 & 289 & 95.4 & 0 & 0.0 & 303 & 100.0 \\
\hline Port Macquarie Base & 2 & 0.3 & 6 & 0.8 & 42 & 5.5 & 712 & 93.4 & 0 & 0.0 & 762 & 100.0 \\
\hline Grafton Base & 3 & 0.7 & 3 & 0.7 & 13 & 3.0 & 421 & 95.7 & 0 & 0.0 & 440 & 100.0 \\
\hline Coffs Harbour & 7 & 0.8 & 2 & 0.2 & 37 & 4.3 & 806 & 94.6 & 0 & 0.0 & 852 & 100.0 \\
\hline Murwillumbah & 3 & 0.7 & 2 & 0.5 & 14 & 3.4 & 388 & 95.3 & 0 & 0.0 & 407 & 100.0 \\
\hline Other Area hospitals & 1 & 0.2 & 0 & 0.0 & 9 & 2.1 & 423 & 97.7 & 0 & 0.0 & 433 & 100.0 \\
\hline ALL HOSPITALS & 45 & 0.8 & 22 & 0.4 & 249 & 4.5 & 5216 & 94.3 & 0 & 0.0 & 5532 & 100.0 \\
\hline \multicolumn{13}{|l|}{ Greater Southern } \\
\hline Griffith Base & 3 & 0.7 & 4 & 0.9 & 20 & 4.5 & 422 & 94.0 & 0 & 0.0 & 449 & 100.0 \\
\hline Wagga Wagga Base & 5 & 0.7 & 6 & 0.8 & 38 & 5.3 & 673 & 93.2 & 0 & 0.0 & 722 & 100.0 \\
\hline Goulburn Base & 4 & 1.4 & 0 & 0.0 & 17 & 5.8 & 271 & 92.8 & 0 & 0.0 & 292 & 100.0 \\
\hline Calvary, Wagga Wagga & 2 & 0.4 & 0 & 0.0 & 37 & 6.5 & 531 & 93.2 & 0 & 0.0 & 570 & 100.0 \\
\hline Moruya & 0 & 0.0 & 3 & 0.9 & 20 & 6.0 & 312 & 93.1 & 0 & 0.0 & 335 & 100.0 \\
\hline Queanbeyan & 0 & 0.0 & 0 & 0.0 & 8 & 2.9 & 269 & 97.1 & 0 & 0.0 & 277 & 100.0 \\
\hline Other Area hospitals & 7 & 0.5 & 4 & 0.3 & 21 & 1.5 & 1336 & 97.7 & 0 & 0.0 & 1368 & 100.0 \\
\hline ALL HOSPITALS & 21 & 0.5 & 17 & 0.4 & 161 & 4.0 & 3814 & 95.0 & 0 & 0.0 & 4013 & 100.0 \\
\hline \multicolumn{13}{|l|}{ Greater Western } \\
\hline Dubbo Base & 18 & 1.5 & 11 & 0.9 & 86 & 7.0 & 1105 & 90.6 & 0 & 0.0 & 1220 & 100.0 \\
\hline Bathurst Base & 3 & 0.5 & 2 & 0.3 & 40 & 6.7 & 548 & 92.4 & 0 & 0.0 & 593 & 100.0 \\
\hline Broken Hill Base & 2 & 0.7 & 1 & 0.4 & 13 & 4.8 & 255 & 94.1 & 0 & 0.0 & 271 & 100.0 \\
\hline Orange Base & 4 & 0.5 & 8 & 1.0 & 52 & 6.5 & 736 & 92.0 & 0 & 0.0 & 800 & 100.0 \\
\hline Mudgee & 0 & 0.0 & 1 & 0.4 & 3 & 1.3 & 228 & 98.3 & 0 & 0.0 & 232 & 100.0 \\
\hline Other Area hospitals & 6 & 0.9 & 1 & 0.2 & 21 & 3.3 & 615 & 95.5 & 1 & 0.2 & 644 & 100.0 \\
\hline ALL HOSPITALS & 33 & 0.9 & 24 & 0.6 & 215 & 5.7 & 3487 & 92.7 & 1 & 0.0 & 3760 & 100.0 \\
\hline TOTAL NSW & 1276 & 1.4 & 826 & 0.9 & 4422 & 4.9 & 84076 & 92.8 & 10 & 0.0 & 90610 & 100.0 \\
\hline
\end{tabular}




\section{Neonatal resuscitation in selected \\ hospitals}

Table 127 shows the type of neonatal resuscitation for individual hospitals where the number of reported confinements exceeded 200 in 2005, totals for all hospitals within each health area, and the NSW total.

\section{TABLE 127}

BIRTHS BY TYPE OF RESUSCITATION AND HOSPITAL, NSW 2005"

Health Area and Hospital

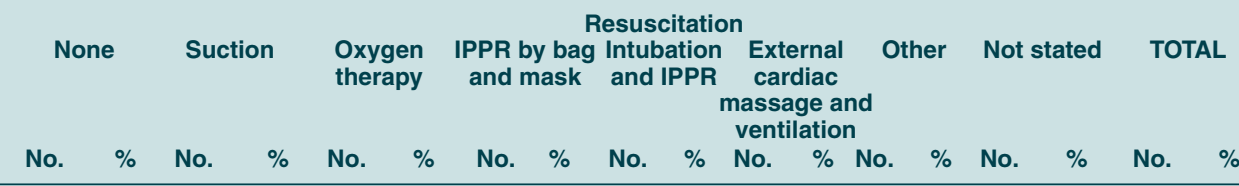

\section{Sydney South West}

Fairfield

Bankstown-Lidcombe

Royal Prince Alfred

Campbelltown

Canterbury

Liverpool

Sydney Southwest Private

Bowral

Other Area hospitals

$\begin{array}{llllll}1168 & 68.7 & 295 & 17.4 & 190 & 11.2\end{array}$

ALL HOSPITALS

$\begin{array}{ll}68 & 3.6\end{array}$

$\begin{array}{llllllll}2711 & 58.5 & 881 & 19.0 & 499 & 10.8 & 496 & 10.7\end{array}$

$\begin{array}{llllllll}1886 & 81.0 & 199 & 8.5 & 209 & 9.0 & 25 & 1.1\end{array}$

$\begin{array}{llllllll}1041 & 66.6 & 404 & 25.8 & 84 & 5.4 & 21 & 1.3\end{array}$

$\begin{array}{llllllll}1993 & 64.8 & 569 & 18.5 & 288 & 9.4 & 179 & 5.8\end{array}$

$\begin{array}{llllllll}703 & 59.2 & 254 & 21.4 & 153 & 12.9 & 70 & 5.9\end{array}$

$\begin{array}{llllllll}562 & 79.0 & 64 & 9.0 & 53 & 7.5 & 29 & 4.1\end{array}$

$\begin{array}{rrrrrrrr}52 & 76.5 & 6 & 8.8 & 5 & 7.4 & 5 & 7.4\end{array}$

South Eastern Sydney \&Illawarra

St. George Private

$\begin{array}{llllllll}1567 & 67.4 & 2937 & 17.1 & 1598 & 9.3 & 936 & 5.5\end{array}$

20.1

$\begin{array}{ll}3 & 0.2\end{array}$

$\begin{array}{ll}0 & 0.0\end{array}$

$2 \quad 0.1$

$\begin{array}{ll}10 & 0.6\end{array}$

301.0

$\begin{array}{rr}1 & 0.1\end{array}$

$\begin{array}{lll}3 & 0.4\end{array}$

$\begin{array}{ll}0 & 0.0\end{array}$

$\begin{array}{ll}51 & 0.3\end{array}$

$\begin{array}{llllllll}2 & 0.1 & 0 & 0.0 & 0 & 0.0 & 1700 & 100.0\end{array}$

$\begin{array}{llll}6 & 0.3 & 0 & 0.0\end{array}$

$\begin{array}{llll}7 & 0.2 & 36 & 0.8\end{array}$

$\begin{array}{llll}6 & 0.3 & 1 & 0.0\end{array}$

$\begin{array}{llll}2 & 0.1 & 1 & 0.1\end{array}$

$\begin{array}{llll}16 & 0.5 & 0 & 0.0\end{array}$

$\begin{array}{llll}0 & 0.0 & 7 & 0.6\end{array}$

$\begin{array}{llll}0 & 0.0 & 0 & 0.0\end{array}$

$\begin{array}{llll}0 & 0.0 & 0 & 0.0\end{array}$

0.01910100 .0

Wollongong

$\begin{array}{llllll}159 & 8.9 & 619 & 34.5 & 461 & 25.7\end{array}$

$\begin{array}{ll}68 & 3.8\end{array}$

$0 \quad 0.0$

$\begin{array}{llll}39 & 0.2 & 45 & 0.3\end{array}$

$\begin{array}{llll}1 & 0.0 & 4631 & 100.0\end{array}$

$\begin{array}{llll}0 & 0.0 & 2328 & 100.0\end{array}$

0.01563100 .0

Hurstville Community

Royal Hospital for Women

$\begin{array}{llllll}1262 & 54.8 & 602 & 26.1 & 337 & 14.6\end{array}$

924.0

$\begin{array}{llllll}29 & 3.2 & 462 & 51.0 & 314 & 34.7\end{array}$

$\begin{array}{ll}33 & 3.6\end{array}$

$\begin{array}{llllll}2899 & 73.6 & 435 & 11.0 & 366 & 9.3\end{array}$

$\begin{array}{rrrrrr}1743 & 74.4 & 194 & 8.3 & 319 & 13.6\end{array}$

$\begin{array}{llllll}580 & 70.9 & 94 & 11.5 & 115 & 14.1\end{array}$

Kareena Private

Prince of Wales Private

$\begin{array}{llllll}38 & 5.9 & 351 & 54.9 & 138 & 21.6\end{array}$

$\begin{array}{lllllll}481 & 29.9 & 770 & 47.9 & 244 & 15.2\end{array}$

$\begin{array}{llllll}661 & 62.7 & 227 & 21.5 & 135 & 12.8\end{array}$

Figtree Private

Other Area hospitals

ALL HOSPITALS

$\begin{array}{llllll}41 & 4.1 & 700 & 70.9 & 206 & 20.9\end{array}$

$\begin{array}{rrrrrr}70 & 81.4 & 4 & 4.7 & 10 & 11.6\end{array}$

$\begin{array}{llllll}7963 & 48.3 & 4458 & 27.1 & 2645 & 16.1\end{array}$

Sydney West

Nepean

Blacktown

Westmead

Westmead Private

Nepean Private

The Hills Private

Hawkesbury

Auburn

Blue Mountains

Other Area hospitals

ALL HOSPITALS

$\begin{array}{llllll}1975 & 56.3 & 744 & 21.2 & 538 & 15.3\end{array}$

$\begin{array}{llllll}1594 & 55.7 & 772 & 27.0 & 385 & 13.4\end{array}$

$\begin{array}{llllll}3177 & 72.1 & 448 & 10.2 & 488 & 11.1\end{array}$

$\begin{array}{llllll}1042 & 57.8 & 389 & 21.6 & 269 & 14.9\end{array}$

$\begin{array}{llllll}448 & 46.7 & 113 & 11.8 & 297 & 30.9\end{array}$

$\begin{array}{lllllll}897 & 68.1 & 208 & 15.8 & 168 & 12.8\end{array}$

$\begin{array}{rrrrrr}561 & 64.9 & 143 & 16.5 & 116 & 13.4\end{array}$

$\begin{array}{llllll}988 & 81.6 & 91 & 7.5 & 63 & 5.2\end{array}$

$\begin{array}{llllll}166 & 61.7 & 63 & 23.4 & 24 & 8.9\end{array}$

$\begin{array}{llllll}33 & 13.5 & 95 & 38.8 & 101 & 41.2\end{array}$

$\begin{array}{ll}77 & 4.5\end{array}$

$\begin{array}{ll}78 & 3.3\end{array}$

$\begin{array}{ll}23 & 2.8\end{array}$

213.3

$\begin{array}{ll}73 & 4.5\end{array}$

$\begin{array}{ll}26 & 2.5\end{array}$

$\begin{array}{ll}37 & 3.7\end{array}$

$\begin{array}{ll}1 & 1.2\end{array}$

$\begin{array}{ll}629 & 3.8\end{array}$

$\begin{array}{rrrrrr}11 & 0.5 & 1 & 0.0 & 0 & 0.0\end{array}$

$\begin{array}{llllll}2 & 0.2 & 1 & 0.1 & 65 & 7.2\end{array}$

$\begin{array}{rrrrrr}45 & 1.1 & 15 & 0.4 & 0 & 0.0\end{array}$

$\begin{array}{llllll}5 & 0.2 & 3 & 0.1 & 0 & 0.0\end{array}$

$\begin{array}{llllll}3 & 0.4 & 3 & 0.4 & 0 & 0.0\end{array}$

$\begin{array}{ll}0 & 0.0\end{array}$

20.1

$\begin{array}{lllr}3 & 0.4 & 0 & 0.0 \\ 0 & 0.0 & 91 & 14.2\end{array}$

$\begin{array}{lll}1 & 0.1\end{array}$

$\begin{array}{ll}3 & 0.3\end{array}$

$\begin{array}{ll}1 & 1.2\end{array}$

$\begin{array}{llll}0 & 0.0 & 17 & 1.1\end{array}$

$\begin{array}{llll}5 & 0.5 & 0 & 0.0\end{array}$

$\begin{array}{llll}0 & 0.0 & 1 & 0.1\end{array}$

$\begin{array}{llll}0 & 0.0 & 0 & 0.0\end{array}$

0.03075100 .0

$0 \quad 0.0 \quad 1188100.0$

0.0711100 .0

$\begin{array}{llll}0 & 0.0 & 68 & 100.0\end{array}$

Northern Sydney \& Central Coas

Gosford

North Shore Private

Royal North Shore

Sydney Adventist

Manly

Hornsby

Mona Vale

Mater, North Sydney

North Gosford Private

Wyong

Other Area hospitals

ALL HOSPITALS

$\begin{array}{rrrrrrrr}1472 & 60.1 & 387 & 15.8 & 450 & 18.4 & 122 & 5.0 \\ 1270 & 48.8 & 806 & 31.0 & 415 & 15.9 & 112 & 4.3 \\ 1287 & 52.0 & 714 & 28.8 & 266 & 10.7 & 151 & 6.1 \\ 642 & 28.8 & 1174 & 52.7 & 250 & 11.2 & 149 & 6.7 \\ 355 & 53.5 & 204 & 30.8 & 83 & 12.5 & 20 & 3.0 \\ 629 & 68.7 & 103 & 11.3 & 148 & 16.2 & 31 & 3.4 \\ 336 & 51.8 & 250 & 38.5 & 49 & 7.6 & 14 & 2.2 \\ 1123 & 49.6 & 582 & 25.7 & 345 & 15.2 & 104 & 4.6 \\ 460 & 52.5 & 189 & 21.6 & 172 & 19.6 & 43 & 4.9 \\ 169 & 75.8 & 26 & 11.7 & 21 & 9.4 & 6 & 2.7 \\ 114 & 85.7 & 14 & 10.5 & 5 & 3.8 & 0 & 0.0\end{array}$

631.8

$\begin{array}{llll}1 & 0.0 & 2 & 0.1\end{array}$

$\begin{array}{lll}96 & 3.4\end{array}$

$173 \quad 3.9$

$\begin{array}{ll}84 & 4.7\end{array}$

$67 \quad 7.0$

$\begin{array}{ll}36 & 2.7\end{array}$

424.9

$\begin{array}{ll}61 & 5.0\end{array}$

$15 \quad 5.6$

$\begin{array}{ll}15 & 6.1\end{array}$

$\begin{array}{ll}9 & 0.3\end{array}$

$\begin{array}{rr}102 & 2.3\end{array}$

$\begin{array}{ll}0 & 0.0\end{array}$

$\begin{array}{ll}9 & 0.9\end{array}$

$\begin{array}{ll}3 & 0.2\end{array}$

$\begin{array}{ll}3 & 0.3\end{array}$

$\begin{array}{ll}4 & 0.3\end{array}$

$\begin{array}{ll}1 & 0.4\end{array}$

10.4

$195 \quad 1.1$

$\begin{array}{lll}8 & 0.3 & 0\end{array}$

\begin{tabular}{llll}
\hline 0 & 0.5 & 0 & 0.0
\end{tabular}

$\begin{array}{llll}4 & 0.2 & 15 & 0.8\end{array}$

$\begin{array}{llll}1 & 0.1 & 25 & 2.6\end{array}$

$\begin{array}{llll}5 & 0.4 & 0 & 0.0\end{array}$

$\begin{array}{llll}0 & 0.0 & 0 & 0.0\end{array}$

$\begin{array}{llll}4 & 0.3 & 0 & 0.0\end{array}$

$\begin{array}{llll}0 & 0.0 & 0 & 0.0\end{array}$

$\begin{array}{llll}0 & 0.0 & 0 & 0.0\end{array}$

$\begin{array}{llllll}4 & 0.2 & 11 & 0.4 & 2 & 0.1\end{array}$

$\begin{array}{lll}4 & 0.2 \\ 1 & 0.0\end{array}$

10.4

$53 \quad 2.1$

$4 \quad 0.2$

$\begin{array}{ll}0 & 0.0 \\ 0 & 0.0\end{array}$

$\begin{array}{llllll}0.1 & 1 & 0.0 & 8 & 0.4\end{array}$

$\begin{array}{llllll}0 & 0.0 & 1 & 0.2 & 0 & 0.0\end{array}$

$\begin{array}{llllll}1 & 0.1 & 3 & 0.3 & 0 & 0.0\end{array}$

$\begin{array}{llllll}0 & 0.0 & 0 & 0.0 & 0 & 0.0\end{array}$

$\begin{array}{llllll}1 & 0.0 & 0 & 0.0 & 109 & 4.8\end{array}$

$\begin{array}{rrrrrr}2 & 0.2 & 1 & 0.1 & 10 & 1.1\end{array}$

$\begin{array}{llllrr}0 & 0.0 & 0 & 0.0 & 1 & 0.4\end{array}$

$\begin{array}{llllll}0 & 0.0 & 0 & 0.0 & 0 & 0.0\end{array}$

$\begin{array}{llllllllllllll}7857 & 50.8 & 4449 & 28.7 & 2204 & 14.2 & 752 & 4.9 & 64 & 0.4 & 21 & 0.1 & 130 & 0.8\end{array}$

$\begin{array}{llll}0 & 0.0 & 1794 & 100.0\end{array}$

$\begin{array}{llll}0 & 0.0 & 2305 & 100.0\end{array}$

$0.0 \quad 906100.0$

$\begin{array}{llll}0 & 0.0 & 3937 & 100.0\end{array}$

$\begin{array}{llll}0 & 0.0 & 2342100.0\end{array}$

$0.0 \quad 818100.0$

$0.0 \quad 639100.0$

$2 \quad 1.4 \quad 1609100.0$

$\begin{array}{lll}0 & 0.0 & 1055100.0\end{array}$

$\begin{array}{lllll}0 & 0.0 & 988 & 100.0\end{array}$

$\begin{array}{llll}0 & 0.0 & 86100.0\end{array}$

$\begin{array}{lll}0 & 0.0 \quad 3507 & 100.0\end{array}$

0.02864100 .0

$\begin{array}{lllll}0 & 0.0 & 4408 & 100.0\end{array}$

0.11804100 .0

0.0960100 .0

$\begin{array}{llll}0 & 0.0 & 1317 & 100.0\end{array}$

$\begin{array}{lll}0 & 0.0 & 865100.0\end{array}$

0.01211100 .0

$\begin{array}{llll}0 & 0.0 & 269100.0\end{array}$

$\begin{array}{rrrr}0 & 0.0 & 245100.0\end{array}$ 
TABLE 127 (continued)

BIRTHS BY TYPE OF RESUSCITATION AND HOSPITAL, NSW 2005*

\begin{tabular}{|c|c|c|c|c|c|c|c|c|c|c|c|c|c|c|c|c|c|}
\hline \multirow[t]{4}{*}{ Health Area and Hospital } & \multirow{3}{*}{\multicolumn{2}{|c|}{ None }} & \multirow{3}{*}{\multicolumn{2}{|c|}{ Suction }} & \multirow{3}{*}{\multicolumn{2}{|c|}{$\begin{array}{l}\text { Oxygen } \\
\text { therapy }\end{array}$}} & \multicolumn{8}{|c|}{ Resuscitatoin } & \multirow{2}{*}{\multicolumn{2}{|c|}{ Not stated }} & \multirow[b]{2}{*}{ TOTAL } \\
\hline & & & & & & & IPPR b & y bag & Intub & $\begin{array}{l}\text { ation } \\
\text { IPPR }\end{array}$ & & & & ther & & & \\
\hline & & & & & & & & & & & $\begin{array}{l}\text { masse } \\
\text { vent }\end{array}$ & $\begin{array}{l}\text { age a } \\
\text { ilatio }\end{array}$ & & & & & \\
\hline & No. & $\%$ & No. & $\%$ & No. & $\%$ & No. & $\%$ & No. & $\%$ & No. & $\%$ & No. & $\%$ & No. & $\%$ & No. \\
\hline Hunter \& New England & & & & & & & & & & & & & & & & & \\
\hline John Hunter & 2185 & 62.9 & 518 & 14.9 & 365 & 10.5 & 363 & 10.4 & 41 & 1.2 & 3 & 0.1 & 0 & 0.0 & 0 & 0.0 & 3475100.0 \\
\hline Tamworth Base & 302 & 37.8 & 155 & 19.4 & 186 & 23.3 & 66 & 8.3 & 6 & 0.8 & 2 & 0.3 & 83 & 10.4 & 0 & 0.0 & 800100.0 \\
\hline Newcastle Private & 1100 & 64.9 & 310 & 18.3 & 220 & 13.0 & 59 & 3.5 & 5 & 0.3 & 2 & 0.1 & 0 & 0.0 & 0 & 0.0 & 1696100.0 \\
\hline Maitland & 1247 & 78.5 & 109 & 6.9 & 172 & 10.8 & 55 & 3.5 & 4 & 0.3 & 2 & 0.1 & 0 & 0.0 & 0 & 0.0 & 1589100.0 \\
\hline Armidale & 116 & 28.2 & 66 & 16.1 & 194 & 47.2 & 27 & 6.6 & 0 & 0.0 & 1 & 0.2 & 6 & 1.5 & 1 & 0.2 & 411100.0 \\
\hline Moree & 108 & 44.8 & 41 & 17.0 & 78 & 32.4 & 12 & 5.0 & 1 & 0.4 & 0 & 0.0 & 1 & 0.4 & 0 & 0.0 & 241100.0 \\
\hline Manning Base & 378 & 53.7 & 148 & 21.0 & 100 & 14.2 & 50 & 7.1 & 2 & 0.3 & 3 & 0.4 & 23 & 3.3 & 0 & 0.0 & 704100.0 \\
\hline Belmont & 216 & 67.1 & 58 & 18.0 & 28 & 8.7 & 20 & 6.2 & 0 & 0.0 & 0 & 0.0 & 0 & 0.0 & 0 & 0.0 & 322100.0 \\
\hline Muswellbrook & 177 & 72.2 & 29 & 11.8 & 25 & 10.2 & 12 & 4.9 & 0 & 0.0 & 0 & 0.0 & 2 & 0.8 & 0 & 0.0 & 245100.0 \\
\hline Inverell & 15 & 6.0 & 12 & 4.8 & 212 & 84.5 & 12 & 4.8 & 0 & 0.0 & 0 & 0.0 & 0 & 0.0 & 0 & 0.0 & 251100.0 \\
\hline Other Area hospitals & 273 & 31.1 & 312 & 35.6 & 244 & 27.8 & 29 & 3.3 & 1 & 0.1 & 5 & 0.6 & 13 & 1.5 & 0 & 0.0 & 877100.0 \\
\hline ALL HOSPITALS & 6117 & 57.6 & 1758 & 16.6 & 1824 & 17.2 & 705 & 6.6 & 60 & 0.6 & 18 & 0.2 & 128 & 1.2 & 1 & 0.0 & 10611100.0 \\
\hline North Coast & & & & & & & & & & & & & & & & & \\
\hline Tweed Heads & 533 & 50.5 & 117 & 11.1 & 282 & 26.7 & 115 & 10.9 & 5 & 0.5 & 3 & 0.3 & 1 & 0.1 & 0 & 0.0 & 1056100.0 \\
\hline Lismore Base & 366 & 28.6 & 461 & 36.0 & 260 & 20.3 & 65 & 5.1 & 9 & 0.7 & 2 & 0.2 & 116 & 9.1 & 0 & 0.0 & 1279100.0 \\
\hline Kempsey & 207 & 68.3 & 17 & 5.6 & 40 & 13.2 & 30 & 9.9 & 3 & 1.0 & 5 & 1.7 & 1 & 0.3 & 0 & 0.0 & 303100.0 \\
\hline Grafton Base & 90 & 20.5 & 253 & 57.5 & 47 & 10.7 & 39 & 8.9 & 8 & 1.8 & 2 & 0.5 & 1 & 0.2 & 0 & 0.0 & 440100.0 \\
\hline Coffs Harbour & 460 & 54.0 & 196 & 23.0 & 125 & 14.7 & 53 & 6.2 & 0 & 0.0 & 1 & 0.1 & 17 & 2.0 & 0 & 0.0 & 852100.0 \\
\hline Port Macquarie Base & 361 & 47.4 & 159 & 20.9 & 144 & 18.9 & 39 & 5.1 & 2 & 0.3 & 4 & 0.5 & 53 & 7.0 & 0 & 0.0 & 762100.0 \\
\hline Murwillumbah & 38 & 9.3 & 228 & 56.0 & 122 & 30.0 & 19 & 4.7 & 0 & 0.0 & 0 & 0.0 & 0 & 0.0 & 0 & 0.0 & 407100.0 \\
\hline Other Area hospitals & 216 & 49.9 & 77 & 17.8 & 97 & 22.4 & 27 & 6.2 & 0 & 0.0 & 1 & 0.2 & 15 & 3.5 & 0 & 0.0 & 433100.0 \\
\hline ALL HOSPITALS & 2271 & 41.1 & 1508 & 27.3 & 1117 & 20.2 & 387 & 7.0 & 27 & 0.5 & 18 & 0.3 & 204 & 3.7 & 0 & 0.0 & 5532100.0 \\
\hline Greater Southern & & & & & & & & & & & & & & & & & \\
\hline Griffith Base & 158 & 35.2 & 120 & 26.7 & 83 & 18.5 & 22 & 4.9 & 2 & 0.4 & 0 & 0.0 & 64 & 14.3 & 0 & 0.0 & 449100.0 \\
\hline Wagga Wagga Base & 221 & 30.6 & 101 & 14.0 & 100 & 13.9 & 65 & 9.0 & 4 & 0.6 & 2 & 0.3 & 229 & 31.7 & 0 & 0.0 & 722100.0 \\
\hline Goulburn Base & 137 & 46.9 & 59 & 20.2 & 79 & 27.1 & 14 & 4.8 & 2 & 0.7 & 1 & 0.3 & 0 & 0.0 & 0 & 0.0 & 292100.0 \\
\hline Moruya & 214 & 63.9 & 54 & 16.1 & 40 & 11.9 & 21 & 6.3 & 2 & 0.6 & 4 & 1.2 & 0 & 0.0 & 0 & 0.0 & 335100.0 \\
\hline Calvary, Wagga Wagga & 257 & 45.1 & 124 & 21.8 & 136 & 23.9 & 43 & 7.5 & 4 & 0.7 & 0 & 0.0 & 6 & 1.1 & 0 & 0.0 & 570100.0 \\
\hline Queanbeyan & 180 & 65.0 & 44 & 15.9 & 38 & 13.7 & 10 & 3.6 & 0 & 0.0 & 0 & 0.0 & 5 & 1.8 & 0 & 0.0 & 277100.0 \\
\hline Other Area hospitals & 839 & 61.3 & 268 & 19.6 & 154 & 11.3 & 73 & 5.3 & 8 & 0.6 & 7 & 0.5 & 19 & 1.4 & 0 & 0.0 & 1368100.0 \\
\hline ALL HOSPITALS & 2006 & 50.0 & 770 & 19.2 & 630 & 15.7 & 248 & 6.2 & 22 & 0.5 & 14 & 0.3 & 323 & 8.0 & 0 & 0.0 & 4013100.0 \\
\hline Greater Western & & & & & & & & & & & & & & & & & \\
\hline Dubbo Base & 601 & 49.3 & 130 & 10.7 & 235 & 19.3 & 53 & 4.3 & 0 & 0.0 & 4 & 0.3 & 196 & 16.1 & 1 & 0.1 & 1220100.0 \\
\hline Bathurst Base & 138 & 23.3 & 280 & 47.2 & 102 & 17.2 & 68 & 11.5 & 3 & 0.5 & 1 & 0.2 & 1 & 0.2 & 0 & 0.0 & 593100.0 \\
\hline Broken Hill Base & 175 & 64.6 & 37 & 13.7 & 43 & 15.9 & 15 & 5.5 & 0 & 0.0 & 1 & 0.4 & 0 & 0.0 & 0 & 0.0 & 271100.0 \\
\hline Orange Base & 442 & 55.3 & 79 & 9.9 & 194 & 24.3 & 74 & 9.3 & 7 & 0.9 & 1 & 0.1 & 3 & 0.4 & 0 & 0.0 & 800100.0 \\
\hline Mudgee & 98 & 42.2 & 58 & 25.0 & 54 & 23.3 & 18 & 7.8 & 2 & 0.9 & 1 & 0.4 & 1 & 0.4 & 0 & 0.0 & 232100.0 \\
\hline Other Area hospitals & 351 & 54.5 & 77 & 12.0 & 165 & 25.6 & 28 & 4.3 & 4 & 0.6 & 5 & 0.8 & 14 & 2.2 & 0 & 0.0 & 644100.0 \\
\hline ALL HOSPITALS & 1805 & 48.0 & 661 & 17.6 & 793 & 21.1 & 256 & 6.8 & 16 & 0.4 & 13 & 0.3 & 215 & 5.7 & 1 & 0.0 & 3760100.0 \\
\hline TOTAL NSW & 50564 & 55.8 & 19610 & 21.6 & 13265 & 14.6 & 4689 & 5.2 & 508 & 0.6 & 196 & 0.21 & 1749 & 1.9 & 29 & 0.0 & 90610100.0 \\
\hline
\end{tabular}




\section{Baby discharge status in selected}

\section{hospitals}

Table 128 shows the discharge status of babies born in hospitals where the number of reported confinements exceeded 200 in 2005, totals for all hospitals within each health area, and the NSW total.

\begin{tabular}{|c|c|c|c|c|c|c|c|c|c|c|c|c|}
\hline \multirow[t]{3}{*}{ Health Area and Hospital } & \multirow{2}{*}{\multicolumn{2}{|c|}{ Discharged }} & \multicolumn{6}{|c|}{ Baby discharge status } & \multirow{2}{*}{\multicolumn{2}{|c|}{ Not stated }} & \multirow{2}{*}{\multicolumn{2}{|c|}{ TOTAL }} \\
\hline & & & & & & atal & Tran & rred & & & & \\
\hline & No. & $\%$ & No. & $\%$ & No. & $\%$ & No. & $\%$ & No. & $\%$ & No. & $\%$ \\
\hline \multicolumn{13}{|l|}{ Sydney South West } \\
\hline Canterbury & 1548 & 99.0 & 3 & 0.2 & 1 & 0.1 & 11 & 0.7 & 0 & 0.0 & 1563 & 100.0 \\
\hline Fairfield & 1667 & 98.1 & 10 & 0.6 & 2 & 0.1 & 21 & 1.2 & 0 & 0.0 & 1700 & 100.0 \\
\hline Campbelltown & 2289 & 98.3 & 16 & 0.7 & 6 & 0.3 & 17 & 0.7 & 0 & 0.0 & 2328 & 100.0 \\
\hline Liverpool & 2835 & 92.2 & 25 & 0.8 & 23 & 0.7 & 192 & 6.2 & 0 & 0.0 & 3075 & 100.0 \\
\hline Bowral & 563 & 79.2 & 4 & 0.6 & 1 & 0.1 & 143 & 20.1 & 0 & 0.0 & 711 & 100.0 \\
\hline Bankstown-Lidcombe & 1872 & 98.0 & 18 & 0.9 & 1 & 0.1 & 19 & 1.0 & 0 & 0.0 & 1910 & 100.0 \\
\hline Sydney Southwest Private & 1172 & 98.7 & 5 & 0.4 & 0 & 0.0 & 11 & 0.9 & 0 & 0.0 & 1188 & 100.0 \\
\hline Royal Prince Alfred & 4442 & 95.9 & 28 & 0.6 & 25 & 0.5 & 136 & 2.9 & 0 & 0.0 & 4631 & 100.0 \\
\hline Other Area hospitals & 67 & 98.5 & 0 & 0.0 & 0 & 0.0 & 1 & 1.5 & 0 & 0.0 & 68 & 100.0 \\
\hline ALL HOSPITALS & 16455 & 95.8 & 109 & 0.6 & 59 & 0.3 & 551 & 3.2 & 0 & 0.0 & 17174 & 100.0 \\
\hline \multicolumn{13}{|c|}{ South Eastern Sydney \&IIlawarra } \\
\hline Sutherland & 1047 & 99.2 & 3 & 0.3 & 1 & 0.1 & 4 & 0.4 & 0 & 0.0 & 1055 & 100.0 \\
\hline Royal Hospital for Women & 3787 & 96.2 & 35 & 0.9 & 31 & 0.8 & 84 & 2.1 & 0 & 0.0 & 3937 & 100.0 \\
\hline St. George & 2303 & 98.3 & 15 & 0.6 & 4 & 0.2 & 20 & 0.9 & 0 & 0.0 & 2342 & 100.0 \\
\hline Wollongong & 1662 & 72.1 & 16 & 0.7 & 9 & 0.4 & 618 & 26.8 & 0 & 0.0 & 2305 & 100.0 \\
\hline Prince of Wales Private & 1576 & 97.9 & 5 & 0.3 & 0 & 0.0 & 7 & 0.4 & 21 & 1.3 & 1609 & 100.0 \\
\hline St. George Private & 1772 & 98.8 & 5 & 0.3 & 0 & 0.0 & 17 & 0.9 & 0 & 0.0 & 1794 & 100.0 \\
\hline Shoalhaven & 740 & 90.5 & 10 & 1.2 & 2 & 0.2 & 66 & 8.1 & 0 & 0.0 & 818 & 100.0 \\
\hline Kareena Private & 628 & 98.3 & 3 & 0.5 & 0 & 0.0 & 8 & 1.3 & 0 & 0.0 & 639 & 100.0 \\
\hline Figtree Private & 973 & 98.5 & 2 & 0.2 & 0 & 0.0 & 13 & 1.3 & 0 & 0.0 & 988 & 100.0 \\
\hline Hurstville Community & 897 & 99.0 & 2 & 0.2 & 0 & 0.0 & 7 & 0.8 & 0 & 0.0 & 906 & 100.0 \\
\hline Other Area hospitals & 78 & 90.7 & 1 & 1.2 & 1 & 1.2 & 6 & 7.0 & 0 & 0.0 & 86 & 100.0 \\
\hline ALL HOSPITALS & 15463 & 93.8 & 97 & 0.6 & 48 & 0.3 & 850 & 5.2 & 21 & 0.1 & 16479 & 100.0 \\
\hline \multicolumn{13}{|l|}{ Sydney West } \\
\hline Hawkesbury & 834 & 96.4 & 2 & 0.2 & 1 & 0.1 & 22 & 2.5 & 6 & 0.7 & 865 & 100.0 \\
\hline The Hills Private & 1296 & 98.4 & 3 & 0.2 & 2 & 0.2 & 16 & 1.2 & 0 & 0.0 & 1317 & 100.0 \\
\hline Nepean & 3319 & 94.6 & 31 & 0.9 & 21 & 0.6 & 135 & 3.8 & 1 & 0.0 & 3507 & 100.0 \\
\hline Blacktown & 2819 & 98.4 & 13 & 0.5 & 3 & 0.1 & 29 & 1.0 & 0 & 0.0 & 2864 & 100.0 \\
\hline Westmead & 4072 & 92.4 & 32 & 0.7 & 24 & 0.5 & 280 & 6.4 & 0 & 0.0 & 4408 & 100.0 \\
\hline Auburn & 1184 & 97.8 & 11 & 0.9 & 1 & 0.1 & 15 & 1.2 & 0 & 0.0 & 1211 & 100.0 \\
\hline Westmead Private & 1775 & 98.4 & 9 & 0.5 & 0 & 0.0 & 20 & 1.1 & 0 & 0.0 & 1804 & 100.0 \\
\hline Nepean Private & 946 & 98.5 & 6 & 0.6 & 1 & 0.1 & 7 & 0.7 & 0 & 0.0 & 960 & 100.0 \\
\hline Blue Mountains & 263 & 97.8 & 1 & 0.4 & 0 & 0.0 & 5 & 1.9 & 0 & 0.0 & 269 & 100.0 \\
\hline Other Area hospitals & 237 & 96.7 & 2 & 0.8 & 0 & 0.0 & 6 & 2.4 & 0 & 0.0 & 245 & 100.0 \\
\hline ALL HOSPITALS & 16745 & 96.0 & 110 & 0.6 & 53 & 0.3 & 535 & 3.1 & 7 & 0.0 & 17450 & 100.0 \\
\hline \multicolumn{13}{|c|}{ Northern Sydney \& Central Coast } \\
\hline Gosford & 2036 & 83.2 & 13 & 0.5 & 2 & 0.1 & 397 & 16.2 & 0 & 0.0 & 2448 & 100.0 \\
\hline Royal North Shore & 2282 & 92.2 & 12 & 0.5 & 23 & 0.9 & 158 & 6.4 & 0 & 0.0 & 2475 & 100.0 \\
\hline Mater, North Sydney & 2251 & 99.3 & 5 & 0.2 & 0 & 0.0 & 10 & 0.4 & 0 & 0.0 & 2266 & 100.0 \\
\hline North Shore Private & 2578 & 99.0 & 8 & 0.3 & 7 & 0.3 & 11 & 0.4 & 0 & 0.0 & 2604 & 100.0 \\
\hline North Gosford Private & 868 & 99.0 & 2 & 0.2 & 0 & 0.0 & 7 & 0.8 & 0 & 0.0 & 877 & 100.0 \\
\hline Manly & 652 & 98.3 & 3 & 0.5 & 1 & 0.2 & 7 & 1.1 & 0 & 0.0 & 663 & 100.0 \\
\hline Wyong & 219 & 98.2 & 0 & 0.0 & 1 & 0.4 & 3 & 1.3 & 0 & 0.0 & 223 & 100.0 \\
\hline Hornsby & 897 & 98.0 & 3 & 0.3 & 2 & 0.2 & 13 & 1.4 & 0 & 0.0 & 915 & 100.0 \\
\hline Mona Vale & 626 & 96.5 & 1 & 0.2 & 0 & 0.0 & 22 & 3.4 & 0 & 0.0 & 649 & 100.0 \\
\hline Sydney Adventist & 2206 & 99.1 & 8 & 0.4 & 0 & 0.0 & 12 & 0.5 & 0 & 0.0 & 2226 & 100.0 \\
\hline Other Area hospitals & 130 & 97.7 & 0 & 0.0 & 0 & 0.0 & 1 & 0.8 & 2 & 1.5 & 133 & 100.0 \\
\hline ALL HOSPITALS & 14745 & 95.3 & 55 & 0.4 & 36 & 0.2 & 641 & 4.1 & 2 & 0.0 & 15479 & 100.0 \\
\hline
\end{tabular}


TABLE 128 (continued)

CONFINEMENTS BY BABY DISCHARGE STATUS AND HOSPITAL, NSW 2005\#

\begin{tabular}{|c|c|c|c|c|c|c|c|c|c|c|c|c|}
\hline \multirow[t]{3}{*}{ Health Area and Hospital } & \multirow{2}{*}{\multicolumn{2}{|c|}{ Discharged }} & \multicolumn{8}{|c|}{ Baby discharge status } & \multirow{2}{*}{\multicolumn{2}{|c|}{ TOTAL }} \\
\hline & & & & orn & \multicolumn{2}{|c|}{$\begin{array}{l}\text { Neonatal } \\
\text { Death }\end{array}$} & \multicolumn{2}{|c|}{ Transferred } & \multicolumn{2}{|c|}{ Not stated } & & \\
\hline & No. & $\%$ & No. & $\%$ & No. & $\%$ & No. & $\%$ & No. & $\%$ & No. & $\%$ \\
\hline \multicolumn{13}{|l|}{ Hunter \& New England } \\
\hline John Hunter & 3140 & 90.4 & 50 & 1.4 & 28 & 0.8 & 257 & 7.4 & 0 & 0.0 & 3475 & 100.0 \\
\hline Manning Base & 668 & 94.9 & 2 & 0.3 & 2 & 0.3 & 32 & 4.5 & 0 & 0.0 & 704 & 100.0 \\
\hline Maitland & 1340 & 84.3 & 8 & 0.5 & 6 & 0.4 & 235 & 14.8 & 0 & 0.0 & 1589 & 100.0 \\
\hline Muswellbrook & 230 & 93.9 & 0 & 0.0 & 1 & 0.4 & 14 & 5.7 & 0 & 0.0 & 245 & 100.0 \\
\hline Newcastle Private & 1566 & 92.3 & 17 & 1.0 & 3 & 0.2 & 110 & 6.5 & 0 & 0.0 & 1696 & 100.0 \\
\hline Belmont & 312 & 96.9 & 1 & 0.3 & 0 & 0.0 & 9 & 2.8 & 0 & 0.0 & 322 & 100.0 \\
\hline Armidale & 387 & 94.2 & 3 & 0.7 & 1 & 0.2 & 20 & 4.9 & 0 & 0.0 & 411 & 100.0 \\
\hline Inverell & 243 & 96.8 & 2 & 0.8 & 1 & 0.4 & 5 & 2.0 & 0 & 0.0 & 251 & 100.0 \\
\hline Tamworth Base & 653 & 81.6 & 6 & 0.8 & 3 & 0.4 & 138 & 17.3 & 0 & 0.0 & 800 & 100.0 \\
\hline Moree & 233 & 96.7 & 5 & 2.1 & 0 & 0.0 & 3 & 1.2 & 0 & 0.0 & 241 & 100.0 \\
\hline Other Area hospitals & 820 & 93.5 & 4 & 0.5 & 1 & 0.1 & 51 & 5.8 & 1 & 0.1 & 877 & 100.0 \\
\hline ALL HOSPITALS & 9592 & 90.4 & 98 & 0.9 & 46 & 0.4 & 874 & 8.2 & 1 & 0.0 & 10611 & 100.0 \\
\hline \multicolumn{13}{|l|}{ North Coast } \\
\hline Grafton Base & 432 & 98.2 & 2 & 0.5 & 0 & 0.0 & 6 & 1.4 & 0 & 0.0 & 440 & 100.0 \\
\hline Coffs Harbour & 783 & 91.9 & 3 & 0.4 & 0 & 0.0 & 66 & 7.7 & 0 & 0.0 & 852 & 100.0 \\
\hline Kempsey & 283 & 93.4 & 3 & 1.0 & 1 & 0.3 & 16 & 5.3 & 0 & 0.0 & 303 & 100.0 \\
\hline Lismore Base & 1072 & 83.8 & 9 & 0.7 & 2 & 0.2 & 196 & 15.3 & 0 & 0.0 & 1279 & 100.0 \\
\hline Tweed Heads & 1035 & 98.0 & 5 & 0.5 & 1 & 0.1 & 15 & 1.4 & 0 & 0.0 & 1056 & 100.0 \\
\hline Port Macquarie Base & 743 & 97.5 & 1 & 0.1 & 1 & 0.1 & 17 & 2.2 & 0 & 0.0 & 762 & 100.0 \\
\hline Murwillumbah & 400 & 98.3 & 1 & 0.2 & 0 & 0.0 & 6 & 1.5 & 0 & 0.0 & 407 & 100.0 \\
\hline Other Area hospitals & 403 & 93.1 & 1 & 0.2 & 0 & 0.0 & 29 & 6.7 & 0 & 0.0 & 433 & 100.0 \\
\hline ALL HOSPITALS & 5151 & 93.1 & 25 & 0.5 & 5 & 0.1 & 351 & 6.3 & 0 & 0.0 & 5532 & 100.0 \\
\hline \multicolumn{13}{|l|}{ Greater Southern } \\
\hline Queanbeyan & 269 & 97.1 & 0 & 0.0 & 0 & 0.0 & 8 & 2.9 & 0 & 0.0 & 277 & 100.0 \\
\hline Moruya & 322 & 96.1 & 1 & 0.3 & 0 & 0.0 & 12 & 3.6 & 0 & 0.0 & 335 & 100.0 \\
\hline Wagga Wagga Base & 636 & 88.1 & 4 & 0.6 & 3 & 0.4 & 79 & 10.9 & 0 & 0.0 & 722 & 100.0 \\
\hline Calvary, Wagga Wagga & 555 & 97.4 & 1 & 0.2 & 1 & 0.2 & 13 & 2.3 & 0 & 0.0 & 570 & 100.0 \\
\hline Goulburn Base & 279 & 95.5 & 4 & 1.4 & 0 & 0.0 & 9 & 3.1 & 0 & 0.0 & 292 & 100.0 \\
\hline Griffith Base & 440 & 98.0 & 3 & 0.7 & 0 & 0.0 & 6 & 1.3 & 0 & 0.0 & 449 & 100.0 \\
\hline Other Area hospitals & 1313 & 96.0 & 3 & 0.2 & 0 & 0.0 & 52 & 3.8 & 0 & 0.0 & 1368 & 100.0 \\
\hline ALL HOSPITALS & 3814 & 95.0 & 16 & 0.4 & 4 & 0.1 & 179 & 4.5 & 0 & 0.0 & 4013 & 100.0 \\
\hline \multicolumn{13}{|l|}{ Greater Western } \\
\hline Dubbo Base & 870 & 71.3 & 13 & 1.1 & 5 & 0.4 & 332 & 27.2 & 0 & 0.0 & 1220 & 100.0 \\
\hline Bathurst Base & 456 & 76.9 & 3 & 0.5 & 0 & 0.0 & 134 & 22.6 & 0 & 0.0 & 593 & 100.0 \\
\hline Broken Hill Base & 263 & 97.0 & 2 & 0.7 & 0 & 0.0 & 6 & 2.2 & 0 & 0.0 & 271 & 100.0 \\
\hline Mudgee & 226 & 97.4 & 0 & 0.0 & 0 & 0.0 & 6 & 2.6 & 0 & 0.0 & 232 & 100.0 \\
\hline Orange Base & 652 & 81.5 & 2 & 0.3 & 0 & 0.0 & 146 & 18.3 & 0 & 0.0 & 800 & 100.0 \\
\hline Other Area hospitals & 575 & 89.3 & 5 & 0.8 & 2 & 0.3 & 62 & 9.6 & 0 & 0.0 & 644 & 100.0 \\
\hline ALL HOSPITALS & 3042 & 80.9 & 25 & 0.7 & $\begin{array}{ll}7 & 0.2\end{array}$ & 686 & 1 & 8.2 & 0 & 0.0 & 3760 & 100.0 \\
\hline Other/Not stated & 110 & 98.2 & 0 & 0.0 & 0 & 0.0 & 1 & 0.9 & 1 & 0.9 & 112 & 100.0 \\
\hline TOTAL NSW & 85117 & 93.9 & 535 & 0.6 & 258 & 0.34 & 668 & 5.2 & 32 & 0.0 & 90610 & 100.0 \\
\hline
\end{tabular}




\section{Postnatal length of stay in selected \\ hospitals}

Table 129 shows the mother's postnatal length of stay in the hospital of birth for hospitals where the number of reported confinements exceeded 200 in 2004, totals for all hospitals within each health area, and the NSW total.

\section{TABLE 129}

AVERAGE MATERNAL POSTNATAL LENGTH OF STAY IN HOSPITAL OF BIRTH, NSW 2000-2004*

\begin{tabular}{|c|c|c|c|c|c|c|c|c|c|c|c|}
\hline \multirow[t]{2}{*}{ Health Area and Hospital } & \multicolumn{5}{|c|}{ Average postnatal length of stay (days) } & \multirow[t]{2}{*}{ Health Area and Hospital } & \multicolumn{5}{|c|}{ Average postnatal length of stay (days) } \\
\hline & 2000 & 2001 & 2002 & 2003 & 2004 & & 2000 & 2001 & 2002 & 2003 & 2004 \\
\hline \multicolumn{6}{|l|}{ Sydney South West } & \multicolumn{6}{|l|}{ Hunter \& New England } \\
\hline Canterbury & 2.8 & 2.7 & 2.9 & 3.0 & 3.0 & Armidale & 3.9 & 3.7 & 3.7 & 3.7 & 3.8 \\
\hline Royal Prince Alfred & 3.6 & 3.7 & 3.8 & 3.3 & 3.9 & Inverell & 3.2 & 3.0 & 3.1 & 2.6 & 3.3 \\
\hline Camden & - & - & - & 2.7 & 2.8 & Moree & 3.6 & 3.3 & 3.5 & 3.5 & 3.4 \\
\hline Fairfield & 2.6 & 2.6 & 2.5 & 2.5 & 2.6 & Tamworth Base & 3.6 & 3.5 & 3.3 & 2.8 & 3.5 \\
\hline Liverpool & 2.8 & 2.7 & 2.6 & 2.1 & 2.8 & Manning Base & 3.6 & 3.1 & 3.0 & 3.1 & 3.2 \\
\hline Campbelltown & 2.5 & 2.5 & 2.6 & 2.3 & 2.5 & Maitland & 2.9 & 2.6 & 2.5 & 2.4 & 2.4 \\
\hline Bankstown/Lidcombe & 2.8 & 2.8 & 2.7 & 2.4 & 2.7 & Muswellbrook & 3.4 & 3.3 & 3.2 & 3.2 & 2.8 \\
\hline Sydney Southwest Private & 4.9 & 4.6 & 4.3 & 3.8 & 4.3 & Belmont & 3.1 & 3.3 & 3.1 & 3.1 & 2.9 \\
\hline Bowral & 2.7 & 2.6 & 2.4 & 1.7 & 2.2 & John Hunter & 3.3 & 3.3 & 3.2 & 2.9 & 3.2 \\
\hline Other Area hospitals & 4.5 & - & - & 5.0 & - & Christo Road Private & 4.9 & 4.9 & 4.7 & 4.5 & 4.6 \\
\hline ALL HOSPITALS & 3.0 & 3.0 & 3.0 & 2.7 & 3.1 & Newcastle Private & 6.0 & 6.8 & 5.2 & 5.8 & 4.3 \\
\hline \multicolumn{6}{|c|}{ South Eastern Sydney \&IIlawarra } & Other Area hospitals & 3.7 & 3.8 & 3.6 & 3.2 & 3.7 \\
\hline Royal Hospital for Women & 3.5 & 3.6 & 3.6 & 3.4 & 3.5 & ALL HOSPITALS & 3.5 & 3.5 & 3.3 & 3.1 & 3.4 \\
\hline St. George & 3.3 & 2.9 & 2.7 & 2.7 & 2.7 & North Coast & & & & & \\
\hline Sutherland & 3.2 & 3.0 & 3.1 & 3.1 & 3.1 & Coffs Harbour & 4.0 & 3.8 & 3.8 & 3.7 & 3.9 \\
\hline Hurstville Community & 4.5 & 4.4 & 4.4 & 4.4 & 4.5 & Grafton Base & 3.8 & 3.5 & 3.6 & 3.6 & 3.6 \\
\hline Kareena Private & 5.7 & 5.2 & 5.0 & 4.8 & 4.5 & Kempsey & 3.6 & 3.2 & 3.0 & 2.0 & 3.1 \\
\hline St. George Private & 5.1 & 5.2 & 4.9 & 4.6 & 4.6 & Lismore Base & 3.2 & 3.1 & 3.2 & 2.9 & 3.5 \\
\hline Prince of Wales Private & 4.9 & 4.6 & 4.8 & 4.8 & 4.6 & Murwillumbah & 3.7 & 3.6 & 3.6 & 3.4 & 3.7 \\
\hline Shoalhaven & 2.6 & 2.5 & 2.3 & 1.4 & 2.5 & Tweed Heads & 3.0 & 3.0 & 3.1 & 3.0 & 3.1 \\
\hline Figtree Private & 5.6 & 5.4 & 5.5 & 5.3 & 5.2 & Port Macquarie Base & 3.8 & 3.7 & 3.8 & 3.5 & 3.7 \\
\hline Other Area hospitals & 2.3 & 2.7 & 2.7 & 2.6 & 2.5 & Other Area hospitals & 3.7 & 3.7 & 3.5 & 3.5 & 3.4 \\
\hline ALL HOSPITALS & 3.7 & 3.8 & 3.7 & 3.6 & 3.6 & ALL HOSPITALS & 3.6 & 3.4 & 3.4 & 3.2 & 3.5 \\
\hline \multicolumn{6}{|l|}{ Sydney West } & \multicolumn{6}{|l|}{ Greater Southern } \\
\hline Auburn & 2.8 & 2.8 & 2.8 & 2.5 & 2.8 & Cooma & 3.9 & 3.9 & 4.0 & 3.8 & 3.8 \\
\hline Blacktown & 3.0 & 3.0 & 2.9 & 2.8 & 3.1 & Goulburn Base & 3.5 & 3.4 & 3.4 & 2.4 & 3.5 \\
\hline Blue Mountains & 3.5 & 3.7 & 3.6 & 3.4 & 3.0 & Moruya & 2.9 & 2.8 & 2.9 & 0.9 & 2.7 \\
\hline Nepean & 3.3 & 3.2 & 3.0 & 2.8 & 3.2 & Queanbeyan & 3.2 & 3.1 & 3.2 & 3.0 & 3.1 \\
\hline Westmead & 3.3 & 3.2 & 3.2 & 3.0 & 3.2 & Griffith Base & 3.1 & 3.1 & 3.1 & 2.5 & 3.2 \\
\hline The Hills Private & 5.2 & 5.0 & 4.8 & 4.7 & 4.6 & Wagga Wagga Base & 3.1 & 2.9 & 3.0 & 2.9 & 2.9 \\
\hline Hawkesbury & 3.3 & 3.2 & 3.4 & 2.6 & 3.4 & Calvary, Wagga Wagga & 4.7 & 4.9 & 4.6 & 4.9 & 5.1 \\
\hline Nepean Private & 4.3 & 4.8 & 4.7 & 4.7 & 4.6 & Other Area hospitals & 3.7 & 3.8 & 3.7 & 3.5 & 3.5 \\
\hline Westmead Private & 4.8 & 4.9 & 4.7 & 4.1 & 4.0 & ALL HOSPITALS & 3.5 & 3.6 & 3.6 & 3.3 & 3.5 \\
\hline Other Area hospitals & 4.3 & 4.0 & 3.1 & 3.5 & 3.5 & Greater Western & & & & & \\
\hline ALL HOSPITALS & 3.4 & 3.5 & 3.4 & 3.2 & 3.4 & $\begin{array}{l}\text { Greater vvestern } \\
\text { Dubbo Base }\end{array}$ & 2.7 & 2.5 & 2.4 & 1.9 & 2.4 \\
\hline \multicolumn{6}{|c|}{ Northern Sydney \& Central Coast } & Bathurst Base & 3.2 & 3.1 & 3.1 & 2.9 & 3.0 \\
\hline Gosford & 2.4 & 2.3 & 2.5 & 2.2 & 2.4 & Cowra & 3.9 & 3.8 & 3.7 & 1.8 & 3.3 \\
\hline Wyong & 2.3 & 2.2 & 2.1 & 2.1 & 2.0 & Orange Base & 3.2 & 3.2 & 3.3 & 3.0 & 3.0 \\
\hline Hornsby & 3.6 & 3.5 & 3.0 & 2.9 & 2.9 & Broken Hill Base & 3.4 & 3.6 & 3.4 & 3.1 & 3.6 \\
\hline Manly & 3.8 & 3.6 & 3.5 & 3.6 & 3.8 & Other Area hospitals & 3.4 & 3.3 & 3.1 & 2.9 & 3.1 \\
\hline Mona Vale & 3.8 & 3.5 & 3.5 & 3.3 & 3.2 & ALL HOSPITALS & 3.1 & 3.0 & 3.0 & 2.5 & 2.9 \\
\hline Royal North Shore & 3.9 & 3.7 & 3.6 & 3.4 & 3.3 & & & & & & \\
\hline Ryde & 3.3 & 3.1 & 3.4 & 3.0 & 3.1 & TOTAL NSW & 3.5 & 3.5 & 3.5 & 3.2 & 3.4 \\
\hline Mater, North Sydney & 5.2 & 4.8 & 4.5 & 4.3 & 4.5 & & & & & & \\
\hline North Shore Private & 4.7 & 4.6 & 4.5 & 4.3 & 4.4 & & & & & & \\
\hline Sydney Adventist & 5.2 & 4.7 & 4.6 & 4.5 & 4.6 & & & & & & \\
\hline North Gosford Private & 5.3 & 4.9 & 4.8 & 4.7 & 4.6 & & & & & & \\
\hline ALL HOSPITALS & 4.1 & 3.9 & 3.9 & 3.7 & 3.8 & & & & & & \\
\hline
\end{tabular}




\section{Induction of labour for other than defined indications, Indicator 1.1}

Definition: The number of patients undergoing induction of labour other than for defined indications (excluding augmentation of labour) as a percentage of the total number of patients undergoing induction of labour for any reason (excluding augmentation of labour).
Table 130 shows aggregate information for hospitals where the reported confinements exceeded 200 in 2005, totals for hospitals within each health area and the NSW total.

\section{TABLE 130}

INDICATOR 1.1: INDUCTION OF LABOUR FOR OTHER THAN DEFINED INDICATIONS BY HOSPITAL, NSW 2001-2005"

\begin{tabular}{|c|c|c|c|c|c|c|c|c|c|c|c|}
\hline Health Area/ Hospital & $\begin{array}{c}2001 \\
\%\end{array}$ & $\begin{array}{c}2002 \\
\%\end{array}$ & $\begin{array}{c}2003 \\
\%\end{array}$ & $\begin{array}{c}2004 \\
\%\end{array}$ & $\begin{array}{c}2005 \\
\%\end{array}$ & Health Area/ Hospital & $\begin{array}{c}2001 \\
\%\end{array}$ & $\begin{array}{c}2002 \\
\%\end{array}$ & $\begin{array}{c}2003 \\
\%\end{array}$ & $\begin{array}{c}2004 \\
\%\end{array}$ & $\begin{array}{c}2004 \\
\%\end{array}$ \\
\hline \multicolumn{6}{|l|}{ Sydney South West } & \multicolumn{6}{|l|}{ Hunter \& New England } \\
\hline Canterbury & 15.2 & 19.3 & 20.6 & 23.0 & 25.4 & Armidale & 36.6 & 35.7 & 51.5 & 38.9 & 21.7 \\
\hline Royal Prince Alfred & 21.9 & 23.6 & 27.8 & 25.6 & 32.3 & Inverell & 42.0 & 38.5 & 52.2 & 52.5 & 54.7 \\
\hline Fairfield & 12.9 & 16.9 & 20.6 & 22.6 & 20.7 & Moree & 34.9 & 36.6 & 49.0 & 32.3 & 31.1 \\
\hline Liverpool & 27.9 & 31.5 & 27.6 & 25.8 & 27.9 & Tamworth Base & 24.9 & 27.2 & 18.4 & 15.3 & 25.4 \\
\hline Campbelltown & 23.5 & 22.3 & 19.9 & 25.3 & 17.8 & Manning Base & 43.3 & 43.9 & 49.0 & 55.2 & 54.8 \\
\hline Bankstown-Lidcombe & 18.3 & 19.4 & 20.2 & 19.5 & 25.4 & Maitland & 29.0 & 29.4 & 29.8 & 30.1 & 26.3 \\
\hline Sydney Southwest Private & 45.1 & 47.9 & 50.2 & 50.6 & 55.4 & Muswellbrook & 27.6 & 27.5 & 16.4 & 28.4 & 21.1 \\
\hline Bowral & 26.3 & 44.1 & 39.5 & 42.5 & 41.4 & Belmont & 27.7 & 27.0 & 24.5 & 27.5 & 30.0 \\
\hline Other Area hospitals & - & - & 21.1 & 23.3 & 38.5 & John Hunter & 24.9 & 27.2 & 24.1 & 27.1 & 27.0 \\
\hline ALL HOSPITALS & 23.8 & 27.0 & 27.8 & 27.9 & 30.2 & Newcastle Private & - & - & - & 40.6 & 41.2 \\
\hline \multicolumn{6}{|c|}{ South Eastern Sydney \&IIlawarra } & Other Area hospitals & 32.7 & 36.3 & 39.8 & 37.6 & 46.2 \\
\hline Royal Hospital for Women & 21.2 & 24.2 & 25.2 & 28.6 & 27.1 & ALL HOSPITALS & 30.1 & 31.7 & 32.7 & 33.6 & 33.7 \\
\hline St. George & 18.6 & 20.9 & 21.2 & 16.9 & 25.1 & \multicolumn{6}{|l|}{ North Coast } \\
\hline Sutherland & 20.6 & 24.2 & 17.9 & 26.3 & 25.6 & Coffs Harbour & 45.5 & 41.5 & 44.0 & 44.6 & 44.7 \\
\hline Hurstville Community & 61.3 & 55.5 & 63.2 & 60.9 & 60.9 & Grafton Base & 25.2 & 28.3 & 23.5 & 11.6 & 21.5 \\
\hline Kareena Private & 60.4 & 62.6 & 64.7 & 71.7 & 70.0 & Kempsey & 26.8 & 26.9 & 17.2 & 22.5 & 25.5 \\
\hline St. George Private & 44.6 & 41.4 & 40.2 & 53.6 & 55.1 & Lismore Base & 19.9 & 19.0 & 21.2 & 15.2 & 18.1 \\
\hline Prince of Wales Private & 49.0 & 40.4 & 40.6 & 48.1 & 44.4 & Murwillumbah & 28.4 & 23.8 & 34.9 & 33.3 & 20.5 \\
\hline Shoalhaven & 31.2 & 30.4 & 30.5 & 34.5 & 29.6 & Tweed Heads & 21.9 & 32.3 & 31.8 & 27.5 & 21.0 \\
\hline Wollongong & 20.6 & 26.0 & 23.3 & 29.8 & 34.0 & Port Macquarie Base & 24.3 & 24.7 & 24.8 & 26.8 & 29.1 \\
\hline Figtree Private & 38.9 & 40.5 & 35.8 & 45.6 & 52.1 & Other Area hospitals & 31.3 & 29.6 & 29.8 & 32.1 & 43.5 \\
\hline Other Area hospitals & 19.0 & 22.7 & 19.5 & 50.0 & 44.4 & ALL HOSPITALS & 28.6 & 28.5 & 29.7 & 26.7 & 26.7 \\
\hline ALL HOSPITALS & 34.1 & 34.3 & 34.2 & 39.4 & 39.8 & \multicolumn{6}{|l|}{ Greater Southern } \\
\hline Sydney West & & & & & & Goulburn Base & 29.6 & 7.1 & 24.0 & 35.3 & 36.7 \\
\hline Auburn & 20.0 & 14.5 & 10.9 & 12.0 & 14.9 & Moruya & 35.0 & 28.0 & 18.8 & 44.8 & 26.3 \\
\hline Blacktown & 24.8 & 34.3 & 30.6 & 25.6 & 27.8 & Queanbeyan & 33.9 & 26.7 & 24.2 & 25.5 & 23.3 \\
\hline Blue Mountains & 26.0 & 17.9 & 26.6 & 20.4 & 31.6 & Griffith Base & 30.9 & 22.4 & 23.9 & 23.8 & 25.3 \\
\hline Nepean & 25.3 & 29.8 & 30.4 & 32.5 & 27.8 & Wagga Wagga Base & 18.6 & 25.1 & 21.4 & 20.8 & 20.0 \\
\hline Westmead & 21.9 & 24.2 & 22.2 & 21.5 & 23.3 & Calvary, Wagga Wagga & 56.5 & 62.9 & 60.3 & 49.5 & 52.1 \\
\hline The Hills Private & 53.3 & 55.0 & 58.7 & 67.2 & 66.0 & Other Area hospitals & 31.6 & 33.0 & 31.8 & 31.0 & 39.7 \\
\hline Hawkesbury & 22.5 & 26.1 & 18.6 & 19.2 & 27.3 & ALL HOSPITALS & 33.6 & 35.6 & 33.8 & 33.0 & 34.6 \\
\hline Nepean Private & 30.7 & 35.2 & 31.6 & 39.9 & 39.4 & \multicolumn{6}{|l|}{ Greater Western } \\
\hline Westmead Private & 33.3 & 37.3 & 50.9 & 50.4 & 55.1 & Dubbo Base & 31.9 & 39.3 & 39.1 & 47.0 & 55.6 \\
\hline Other Area hospitals & 28.6 & 25.0 & 28.1 & 25.5 & 28.1 & Mudgee & 21.7 & 14.7 & 25.0 & 25.9 & 31.7 \\
\hline ALL HOSPITALS & 28.5 & 32.5 & 33.4 & 34.3 & 34.7 & Bathurst Base & 30.0 & 15.0 & 18.4 & 12.1 & 17.4 \\
\hline \multicolumn{6}{|c|}{ Northern Sydney \& Central Coast } & Orange Base & 22.8 & 17.5 & 25.9 & 29.6 & 32.2 \\
\hline Gosford & 22.5 & 25.2 & 21.3 & 23.9 & 21.8 & Broken Hill Base & 24.4 & 15.9 & 26.7 & 35.7 & 49.2 \\
\hline Wyong & 11.1 & 12.5 & 25.0 & 33.3 & 33.3 & Other Area hospitals & 32.0 & 38.3 & 37.8 & 22.8 & 26.8 \\
\hline Hornsby & 25.9 & 29.7 & 32.5 & 34.1 & 27.9 & ALL HOSPITALS & 29.0 & 29.3 & 32.2 & 33.7 & 41.0 \\
\hline Manly & 31.0 & 24.9 & 19.7 & 22.2 & 25.5 & \multirow{9}{*}{ TOTAL NSW } & \multirow[t]{9}{*}{31.7} & \multirow{9}{*}{33.1} & \multirow[t]{9}{*}{33.5} & \multirow[t]{9}{*}{35.1} & \multirow{9}{*}{36.2} \\
\hline Mona Vale & 36.8 & 26.3 & 25.3 & 28.7 & 32.5 & & & & & & \\
\hline Royal North Shore & 26.3 & 24.1 & 25.8 & 22.1 & 23.4 & & & & & & \\
\hline Mater, North Sydney & 42.5 & 47.5 & 45.9 & 50.8 & 52.5 & & & & & & \\
\hline North Shore Private & 48.8 & 47.5 & 48.4 & 51.8 & 50.9 & & & & & & \\
\hline Sydney Adventist & 65.9 & 57.2 & 61.3 & 67.8 & 64.4 & & & & & & \\
\hline North Gosford Private & 51.5 & 52.6 & 60.8 & 60.2 & 65.7 & & & & & & \\
\hline Other Area hospitals & 27.0 & 30.7 & 14.3 & 21.4 & - & & & & & & \\
\hline ALL HOSPITALS & 42.1 & 41.3 & 41.0 & 43.6 & 44.0 & & & & & & \\
\hline
\end{tabular}

Source: NSW Midwives Data Collection. Centre for Epidemiology and Research, NSW Health Department.

\# Hospitals with more than 200 deliveries are identified individually. All hospitals include all public and private hospitals. Defined indications include: diabetes, hypertensive disease, fetal distress, fetal death, chorioamnionitis, blood group isoimmunisation, prelabour rupture of membranes, prolonged pregnancy (41 or more weeks), and suspected intrauterine growth retardation. 


\section{Induction of labour for other than defined indications, Indicator $\mathbf{1 . 2}$}

Definition: The number of patients undergoing induction of labour other than for defined indications (excluding augmentation of labour) as a percentage of the total number of patients delivering (excluding augmentation of labour).
Table 131 shows aggregate information for hospitals where the reported confinements exceeded 200 in 2005, totals for hospitals within each health area, and the NSW total.

\section{TABLE 131}

INDICATOR 1.2: INDUCTION OF LABOUR FOR OTHER THAN DEFINED INDICATIONS BY HOSPITAL, NSW 2001-2005"

\begin{tabular}{|c|c|c|c|c|c|c|c|c|c|c|c|}
\hline Health Area/ Hospital & $\begin{array}{c}2001 \\
\%\end{array}$ & $\begin{array}{c}2002 \\
\%\end{array}$ & $\begin{array}{c}2003 \\
\%\end{array}$ & $\begin{array}{c}2004 \\
\%\end{array}$ & $\begin{array}{c}2005 \\
\%\end{array}$ & Health Area/ Hospital & $\begin{array}{c}2001 \\
\%\end{array}$ & $\begin{array}{c}2002 \\
\%\end{array}$ & $\begin{array}{c}2003 \\
\%\end{array}$ & $\begin{array}{c}2004 \\
\%\end{array}$ & $\begin{array}{c}2005 \\
\%\end{array}$ \\
\hline \multicolumn{6}{|l|}{ Sydney South West } & \multicolumn{6}{|l|}{ Hunter \& New England } \\
\hline Canterbury & 3.2 & 4.3 & 4.5 & 4.8 & 4.6 & Armidale & 11.1 & 13.9 & 15.7 & 12.0 & 6.9 \\
\hline Royal Prince Alfred & 4.6 & 5.2 & 5.9 & 5.3 & 7.0 & Inverell & 11.2 & 11.5 & 17.1 & 18.1 & 19.4 \\
\hline Fairfield & 2.6 & 3.2 & 3.9 & 4.4 & 4.0 & Moree & 9.4 & 10.3 & 13.0 & 10.3 & 7.9 \\
\hline Liverpool & 6.8 & 7.5 & 7.0 & 5.7 & 6.1 & Tamworth Base & 6.9 & 8.3 & 5.4 & 4.3 & 7.3 \\
\hline Campbelltown & 5.9 & 5.3 & 5.3 & 7.5 & 4.2 & Manning Base & 10.2 & 11.4 & 14.8 & 17.0 & 15.5 \\
\hline Bankstown-Lidcombe & 3.5 & 4.1 & 4.0 & 4.4 & 5.3 & Maitland & 6.5 & 8.5 & 6.5 & 6.9 & 6.8 \\
\hline Sydney Southwest Private & 17.3 & 19.1 & 20.1 & 19.1 & 19.3 & Muswellbrook & 7.8 & 6.3 & 4.4 & 8.5 & 5.0 \\
\hline Bowral & 6.5 & 11.6 & 10.7 & 11.6 & 12.4 & Belmont & 7.3 & 7.3 & 6.2 & 6.5 & 5.6 \\
\hline Other Area hospitals & - & - & 3.6 & 4.4 & 7.4 & John Hunter & 5.6 & 6.8 & 5.8 & 6.3 & 6.9 \\
\hline ALL HOSPITALS & 5.5 & 6.4 & 6.6 & 6.6 & 6.8 & Newcastle Private & - & - & - & 12.4 & 12.5 \\
\hline \multicolumn{6}{|c|}{ South Eastern Sydney \&IIlawarra } & Other Area hospitals & 9.1 & 10.5 & 11.8 & 10.3 & 14.5 \\
\hline Royal Hospital for Women & 4.4 & 5.5 & 5.6 & 5.8 & 5.8 & ALL HOSPITALS & 7.5 & 8.7 & 8.6 & 8.9 & 9.2 \\
\hline St. George & 3.5 & 4.2 & 4.2 & 3.2 & 5.1 & \multicolumn{6}{|l|}{ North Coast } \\
\hline Sutherland & 5.4 & 6.3 & 4.1 & 6.7 & 6.8 & Coffs Harbour & 12.5 & 10.4 & 12.0 & 10.0 & 10.9 \\
\hline Hurstville Community & 20.0 & 16.0 & 18.9 & 17.3 & 18.8 & Grafton Base & 6.4 & 6.8 & 6.0 & 2.8 & 5.3 \\
\hline Kareena Private & 22.8 & 23.6 & 22.5 & 26.7 & 24.4 & Kempsey & 6.7 & 5.8 & 4.0 & 5.0 & 7.9 \\
\hline St. George Private & 12.8 & 10.0 & 10.4 & 14.9 & 15.3 & Lismore Base & 4.3 & 4.6 & 4.8 & 3.6 & 4.0 \\
\hline Prince of Wales Private & 11.2 & 10.1 & 9.1 & 11.7 & 12.0 & Murwillumbah & 6.7 & 5.9 & 8.1 & 7.5 & 5.7 \\
\hline Shoalhaven & 6.3 & 6.1 & 5.6 & 5.8 & 5.2 & Tweed Heads & 5.2 & 7.3 & 7.5 & 6.8 & 5.3 \\
\hline Wollongong & 5.8 & 7.1 & 5.5 & 7.2 & 9.8 & Port Macquarie Base & 6.5 & 7.0 & 5.5 & 5.7 & 6.9 \\
\hline Figtree Private & 13.1 & 14.1 & 13.9 & 17.7 & 18.8 & Other Area hospitals & 6.1 & 6.6 & 5.3 & 6.1 & 6.9 \\
\hline Other Area hospitals & 4.3 & 4.3 & 4.4 & 10.4 & 14.0 & ALL HOSPITALS & 6.5 & 6.7 & 6.7 & 6.0 & 6.4 \\
\hline ALL HOSPITALS & 8.5 & 8.6 & 8.4 & 9.6 & 10.2 & \multicolumn{6}{|l|}{ Greater Southern } \\
\hline Sydney West & & & & & & Goulburn Base & 2.5 & 0.7 & 2.0 & 2.1 & 3.8 \\
\hline Auburn & 3.6 & 2.6 & 1.9 & 2.1 & 3.1 & Moruya & 4.2 & 4.5 & 3.3 & 10.4 & 6.4 \\
\hline Blacktown & 6.4 & 8.4 & 6.7 & 5.7 & 6.4 & Queanbeyan & 5.9 & 4.7 & 5.9 & 5.3 & 6.1 \\
\hline Blue Mountains & 5.0 & 5.0 & 6.7 & 4.0 & 6.7 & Griffith Base & 6.0 & 4.9 & 4.7 & 4.4 & 5.2 \\
\hline Nepean & 6.7 & 7.8 & 8.7 & 8.6 & 8.5 & Wagga Wagga Base & 4.6 & 6.0 & 4.7 & 4.9 & 4.4 \\
\hline Westmead & 4.2 & 4.7 & 4.2 & 4.5 & 5.1 & Calvary, Wagga Wagga & 20.8 & 22.5 & 19.6 & 16.1 & 13.7 \\
\hline The Hills Private & 19.7 & 20.6 & 22.6 & 25.6 & 27.8 & Other Area hospitals & 8.9 & 9.3 & 8.9 & 8.9 & 11.2 \\
\hline Hawkesbury & 5.5 & 5.7 & 3.7 & 4.2 & 7.8 & ALL HOSPITALS & 8.2 & 9.0 & 8.2 & 8.2 & 8.4 \\
\hline Nepean Private & 10.5 & 10.3 & 10.0 & 12.6 & 12.6 & \multicolumn{6}{|l|}{ Greater Western } \\
\hline Westmead Private & 9.1 & 10.4 & 15.3 & 14.8 & 17.1 & Dubbo Base & 10.4 & 10.8 & 10.9 & 13.7 & 21.1 \\
\hline Other Area hospitals & 6.0 & 6.0 & 7.6 & 6.9 & 7.4 & Mudgee & 4.6 & 2.3 & 4.9 & 3.7 & 8.6 \\
\hline ALL HOSPITALS & 7.1 & 8.0 & 8.3 & 8.6 & 9.4 & Bathurst Base & 5.2 & 2.8 & 3.3 & 2.1 & 3.4 \\
\hline \multicolumn{6}{|c|}{ Northern Sydney \& Central Coast } & Orange Base & 5.6 & 4.1 & 6.5 & 8.0 & 8.5 \\
\hline Gosford & 5.7 & 6.6 & 5.2 & 5.2 & 5.3 & Broken Hill Base & 3.7 & 2.7 & 2.9 & 3.9 & 10.7 \\
\hline Wyong & 0.3 & 0.3 & 0.8 & 1.8 & 0.9 & Other Area hospitals & 6.4 & 7.5 & 7.3 & 5.0 & 5.8 \\
\hline Hornsby & 7.3 & 7.3 & 7.5 & 7.6 & 5.7 & ALL HOSPITALS & 7.0 & 6.6 & 7.3 & 7.9 & 11.5 \\
\hline Manly & 9.0 & 6.5 & 4.9 & 6.0 & 6.1 & \multirow{9}{*}{ TOTAL NSW } & \multirow{9}{*}{7.9} & \multirow{9}{*}{8.3} & \multirow[t]{9}{*}{8.2} & \multirow{9}{*}{8.6} & \multirow{9}{*}{9.2} \\
\hline Mona Vale & 10.1 & 7.2 & 6.3 & 5.7 & 8.4 & & & & & & \\
\hline Royal North Shore & 6.0 & 5.1 & 5.8 & 4.9 & 5.0 & & & & & & \\
\hline Mater, North Sydney & 11.4 & 13.1 & 11.3 & 13.6 & 13.3 & & & & & & \\
\hline North Shore Private & 14.1 & 13.2 & 12.7 & 12.9 & 12.2 & & & & & & \\
\hline Sydney Adventist & 23.1 & 18.3 & 19.1 & 20.7 & 21.9 & & & & & & \\
\hline North Gosford Private & 15.9 & 15.8 & 18.2 & 16.8 & 25.8 & & & & & & \\
\hline Other Area hospitals & 6.6 & 6.5 & 2.7 & 2.3 & 0.0 & & & & & & \\
\hline ALL HOSPITALS & 11.6 & 11.0 & 10.3 & 10.6 & 11.2 & & & & & & \\
\hline
\end{tabular}




\section{Vaginal delivery following primary caesarean section, Indicator 2.1}

Definition: The number of patients delivering vaginally following previous primary caesarean section as a percentage of the total number of patients delivering who have had a previous primary caesarean section with no intervening pregnancies greater than 20 weeks gestation.
Table 132 shows aggregate information for hospitals where the reported confinements exceeded 200 in 2005, totals for hospitals within each health area, and the NSW total.

\section{TABLE 132}

INDICATOR 2.1: VAGINAL DELIVERY AFTER CAESAREAN SECTION BY HOSPITAL, NSW 2001-2005"

\begin{tabular}{|c|c|c|c|c|c|c|c|c|c|c|c|}
\hline Health Area/ Hospital & $\begin{array}{c}2001 \\
\%\end{array}$ & $\begin{array}{c}2002 \\
\%\end{array}$ & $\begin{array}{c}2003 \\
\%\end{array}$ & $\begin{array}{c}2004 \\
\%\end{array}$ & $\begin{array}{c}2005 \\
\%\end{array}$ & Health Area/ Hospital & $\begin{array}{c}2001 \\
\%\end{array}$ & $\begin{array}{c}2002 \\
\%\end{array}$ & $\begin{array}{c}2003 \\
\%\end{array}$ & $\begin{array}{c}2004 \\
\%\end{array}$ & $\begin{array}{c}2005 \\
\%\end{array}$ \\
\hline \multicolumn{6}{|l|}{ Sydney South West } & \multicolumn{6}{|l|}{ Hunter \& New England } \\
\hline Canterbury & 34.3 & 24.8 & 22.1 & 27.5 & 19.9 & Armidale & 19.0 & 46.4 & 33.3 & 25.6 & 14.7 \\
\hline Royal Prince Alfred & 26.6 & 23.5 & 22.5 & 22.5 & 18.6 & Inverell & 8.3 & 8.3 & 9.5 & 11.1 & 7.1 \\
\hline Fairfield & 17.4 & 34.1 & 18.6 & 20.2 & 2.4 & Moree & 35.7 & 33.3 & 34.6 & 13.6 & 23.8 \\
\hline Liverpool & 22.6 & 20.8 & 13.4 & 15.5 & 20.3 & Tamworth Base & 13.3 & 24.3 & 8.3 & 14.8 & 16.5 \\
\hline Campbelltown & 18.8 & 16.7 & 24.7 & 18.1 & 17.9 & Manning Base & 18.4 & 40.8 & 35.7 & 21.7 & 14.9 \\
\hline Bankstown-Lidcombe & 20.6 & 25.6 & 20.5 & 18.9 & 20.0 & Maitland & 14.0 & 11.0 & 16.4 & 10.9 & 15.0 \\
\hline Sydney Southwest Private & 10.8 & 11.6 & 12.6 & 9.8 & 4.3 & Muswellbrook & 33.3 & 27.8 & 25.0 & 46.2 & 13.8 \\
\hline Bowral & 22.4 & 25.0 & 10.4 & 24.5 & 9.6 & Belmont & 26.2 & 14.5 & 11.8 & 19.6 & 18.2 \\
\hline Other Area hospitals & - & - & 50.0 & - & & John Hunter & 25.2 & 28.0 & 25.9 & 21.9 & 16.7 \\
\hline ALL HOSPITALS & 22.8 & 22.1 & 19.2 & 19.6 & 16.1 & Newcastle Private & - & - & - & 7.6 & 12.4 \\
\hline \multicolumn{6}{|c|}{ South Eastern Sydney \&IIlawarra } & Other Area hospitals & 11.8 & 10.9 & 11.6 & 10.4 & 9.5 \\
\hline Royal Hospital for Women & 11.9 & 13.1 & 13.3 & 10.6 & 8.9 & ALL HOSPITALS & 17.7 & 20.1 & 19.5 & 16.1 & 14.6 \\
\hline St. George & 12.9 & 19.7 & 22.2 & 15.2 & 14.8 & \multicolumn{6}{|l|}{ North Coast } \\
\hline Sutherland & 22.4 & 14.3 & 15.7 & 16.2 & 15.4 & Coffs Harbour & 13.8 & 13.5 & 9.5 & 13.1 & 10.7 \\
\hline Hurstville Community & 9.6 & 7.1 & 6.7 & 6.1 & 3.6 & Grafton Base & 22.2 & 17.5 & 9.1 & 16.7 & 6.3 \\
\hline Kareena Private & 4.1 & 4.1 & 2.8 & 3.0 & 1.6 & Kempsey & 33.3 & 55.0 & 40.7 & 15.4 & 16.7 \\
\hline St. George Private & 11.4 & 11.6 & 6.8 & 4.4 & 6.8 & Lismore Base & 31.3 & 28.4 & 17.6 & 24.8 & 12.0 \\
\hline Prince of Wales Private & 6.7 & 7.9 & 4.6 & 3.5 & 5.5 & Murwillumbah & 13.8 & 22.4 & 11.5 & 9.3 & 17.6 \\
\hline Shoalhaven & 18.4 & 19.1 & 23.4 & 15.2 & 8.7 & Tweed Heads & 38.3 & 26.3 & 16.0 & 21.1 & 16.2 \\
\hline Wollongong & 26.7 & 25.2 & 21.6 & 31.0 & 14.9 & Port Macquarie Base & 20.0 & 14.5 & 14.3 & 21.5 & 13.7 \\
\hline Figtree Private & 11.5 & 12.8 & 7.5 & 11.6 & 16.0 & Other Area hospitals & 14.6 & 14.3 & 13.5 & 19.5 & 33.3 \\
\hline Other Area hospitals & 13.6 & 12.5 & 18.8 & 100.0 & 0.0 & ALL HOSPITALS & 24.0 & 21.7 & 15.1 & 18.6 & 13.4 \\
\hline ALL HOSPITALS & 12.4 & 12.9 & 11.7 & 11.0 & 9.3 & \multicolumn{6}{|l|}{ Greater Southern } \\
\hline Sydney West & & & & & & Goulburn Base & 36.4 & 36.7 & 13.0 & 28.9 & 19.4 \\
\hline Auburn & 31.7 & 26.5 & 30.0 & 6.8 & 0.0 & Moruya & 18.2 & 20.0 & 0.0 & 16.7 & 11.4 \\
\hline Blacktown & 29.3 & 23.2 & 21.8 & 18.0 & 22.9 & Queanbeyan & 26.9 & 30.0 & 21.7 & 52.4 & 15.8 \\
\hline Blue Mountains & 39.1 & 12.5 & 33.3 & 33.3 & 20.0 & Griffith Base & 32.1 & 9.8 & 24.3 & 25.6 & 9.8 \\
\hline Nepean & 22.8 & 18.6 & 16.9 & 16.6 & 14.0 & Wagga Wagga Base & 26.8 & 16.0 & 17.5 & 17.8 & 15.5 \\
\hline Westmead & 28.5 & 28.2 & 20.9 & 17.5 & 19.1 & Calvary, Wagga Wagga & 16.3 & 18.2 & 15.6 & 19.7 & 17.2 \\
\hline The Hills Private & 15.1 & 14.6 & 11.2 & 11.4 & 10.8 & Other Area hospitals & 16.2 & 20.8 & 11.8 & 19.1 & 18.0 \\
\hline Hawkesbury & 28.6 & 26.3 & 14.8 & 14.5 & 16.9 & ALL HOSPITALS & 22.5 & 19.8 & 15.0 & 22.6 & 16.1 \\
\hline Nepean Private & 19.8 & 16.7 & 8.4 & 11.5 & 6.1 & \multicolumn{6}{|l|}{ Greater Western } \\
\hline Westmead Private & 18.5 & 22.5 & 17.9 & 16.6 & 9.6 & Dubbo Base & 37.3 & 29.5 & 34.4 & 31.1 & 21.3 \\
\hline Other Area hospitals & 14.3 & 5.3 & 20.8 & 23.1 & 21.6 & Mudgee & 11.8 & 10.0 & 9.5 & 11.8 & 0.0 \\
\hline ALL HOSPITALS & 24.7 & 22.1 & 18.4 & 15.8 & 14.8 & Bathurst Base & 15.0 & 7.7 & 14.8 & 17.8 & 12.2 \\
\hline \multicolumn{6}{|c|}{ Northern Sydney \& Central Coast } & Orange Base & 18.8 & 18.5 & 20.8 & 16.3 & 12.3 \\
\hline Gosford & 23.4 & 22.7 & 16.9 & 10.5 & 13.9 & Broken Hill Base & 12.5 & 22.2 & 26.3 & 32.0 & 16.0 \\
\hline Wyong & 100.0 & - & - & - & - & Other Area hospitals & 14.7 & 19.2 & 8.1 & 21.3 & 16.4 \\
\hline Hornsby & 9.6 & 6.2 & 12.7 & 11.0 & 9.0 & ALL HOSPITALS & 21.4 & 18.8 & 20.4 & 22.2 & 15.0 \\
\hline Manly & 14.3 & 4.4 & 7.0 & 12.1 & 10.6 & \multirow{9}{*}{ TOTAL NSW } & \multirow[t]{9}{*}{18.3} & \multirow[t]{9}{*}{17.2} & \multirow[t]{9}{*}{14.8} & \multirow[t]{9}{*}{14.6} & \multirow[t]{9}{*}{12.4} \\
\hline Mona Vale & 6.8 & 11.5 & 12.9 & 11.4 & 13.0 & & & & & & \\
\hline Royal North Shore & 13.8 & 7.5 & 11.2 & 16.1 & 10.6 & & & & & & \\
\hline Mater, North Sydney & 7.8 & 5.8 & 6.4 & 5.5 & 7.3 & & & & & & \\
\hline North Shore Private & 8.5 & 6.6 & 6.8 & 5.4 & 4.2 & & & & & & \\
\hline Sydney Adventist & 9.9 & 7.0 & 3.6 & 7.1 & 3.2 & & & & & & \\
\hline North Gosford Private & 9.1 & 6.2 & 3.8 & 8.0 & 3.6 & & & & & & \\
\hline Other Area hospitals & 12.1 & 23.3 & 5.3 & 5.3 & . & & & & & & \\
\hline ALL HOSPITALS & 11.4 & 9.1 & 7.8 & 8.3 & 7.2 & & & & & & \\
\hline
\end{tabular}




\section{Primary caesarean section for failure to progress, Indicator 3.1}

Definition: The number of patients undergoing primary Caesarean section for failure to progress after a period of labour with cervical dilation of $3 \mathrm{~cm}$ or less as a percentage of the total number of patients undergoing primary non-elective Caesarean section.
Table 133 shows aggregate information for hospitals where the reported confinements exceeded 200 in 2005, totals for hospitals within each health area, and the NSW total.

\section{TABLE 133}

INDICATOR 3.1: PRIMARY CAESAREAN SECTION FOR FAILURE TO PROGRESS BY HOSPITAL, NSW 2001-2005\#

\begin{tabular}{|c|c|c|c|c|c|c|c|c|c|c|c|}
\hline Health Area/ Hospital & $\begin{array}{c}2001 \\
\%\end{array}$ & $\begin{array}{c}2002 \\
\%\end{array}$ & $\begin{array}{c}2003 \\
\%\end{array}$ & $\begin{array}{c}2004 \\
\%\end{array}$ & $\begin{array}{c}2005 \\
\%\end{array}$ & Health Area/ Hospital & $\begin{array}{c}2001 \\
\%\end{array}$ & $\begin{array}{c}2002 \\
\%\end{array}$ & $\begin{array}{c}2003 \\
\%\end{array}$ & $\begin{array}{c}2004 \\
\%\end{array}$ & $\begin{array}{c}2005 \\
\%\end{array}$ \\
\hline \multicolumn{6}{|l|}{ Sydney South West } & \multicolumn{6}{|l|}{ Hunter \& New England } \\
\hline Canterbury & 13.7 & 9.1 & 9.1 & 10.1 & 3.4 & Armidale & 2.9 & 5.4 & 0.0 & 6.7 & 4.5 \\
\hline Royal Prince Alfred & 5.7 & 6.5 & 8.5 & 5.5 & 6.1 & Inverell & 0.0 & 12.5 & 0.0 & 12.5 & 15.0 \\
\hline Fairfield & 10.0 & 13.0 & 11.5 & 10.3 & 9.7 & Moree & 0.0 & 12.0 & 12.5 & 26.7 & 4.5 \\
\hline Liverpool & 9.2 & 12.4 & 5.0 & 9.1 & 5.7 & Tamworth Base & 15.4 & 9.5 & 7.1 & 3.4 & 4.7 \\
\hline Campbelltown & 7.8 & 7.0 & 11.5 & 8.6 & 9.8 & Manning Base & 17.1 & 14.0 & 15.7 & 15.8 & 18.5 \\
\hline Bankstown-Lidcombe & 8.0 & 3.4 & 6.2 & 16.1 & 6.2 & Maitland & 8.7 & 8.0 & 10.0 & 9.8 & 9.2 \\
\hline Sydney Southwest Private & 13.2 & 18.1 & 6.3 & 11.4 & 4.3 & Muswellbrook & 14.3 & 4.8 & 0.0 & 0.0 & 19.0 \\
\hline Bowral & 7.7 & 8.1 & 18.2 & 4.8 & 4.8 & Belmont & 8.3 & 11.9 & 18.5 & 4.5 & 0.0 \\
\hline Other Area hospitals & - & - & 16.3 & 14.3 & 0.0 & John Hunter & 8.7 & 13.1 & 9.2 & 10.3 & 11.3 \\
\hline ALL HOSPITALS & 8.4 & 8.8 & 8.8 & 8.8 & 6.3 & Newcastle Private & - & - & - & 1.1 & 5.3 \\
\hline \multicolumn{6}{|c|}{ South Eastern Sydney \&IIlawarra } & Other Area hospitals & 13.7 & 8.0 & 5.4 & 8.2 & 14.5 \\
\hline Royal Hospital for Women & 4.0 & 5.4 & 6.3 & 6.7 & 8.5 & ALL HOSPITALS & 10.2 & 10.4 & 8.8 & 8.4 & 9.6 \\
\hline St. George & 9.3 & 6.6 & 9.8 & 4.6 & 8.7 & North Coast & & & & & \\
\hline Sutherland & 12.9 & 16.1 & 9.4 & 8.2 & 15.5 & Coffs Harbour & 13.6 & 16.4 & 15.4 & 7.5 & 14.1 \\
\hline Hurstville Community & 20.5 & 17.1 & 16.4 & 10.9 & 11.5 & Grafton Base & 16.3 & 10.4 & 5.8 & 21.4 & 2.2 \\
\hline Kareena Private & 14.5 & 18.5 & 11.1 & 8.9 & 10.8 & Kempsey & 11.8 & 0.0 & 11.5 & 6.3 & 7.7 \\
\hline St. George Private & 13.5 & 19.9 & 13.3 & 13.5 & 16.5 & Lismore Base & 10.1 & 7.8 & 9.4 & 12.0 & 4.6 \\
\hline Prince of Wales Private & 11.0 & 9.4 & 7.7 & 11.3 & 9.8 & Murwillumbah & 7.9 & 19.1 & 19.1 & 20.0 & 19.1 \\
\hline Shoalhaven & 8.8 & 9.2 & 9.7 & 13.5 & 7.2 & Tweed Heads & 16.7 & 10.7 & 14.5 & 7.7 & 11.2 \\
\hline Wollongong & 7.1 & 6.9 & 7.3 & 5.3 & 7.1 & Port Macquarie Base & 6.8 & 5.8 & 2.3 & 5.1 & 7.6 \\
\hline Figtree Private & 15.8 & 11.5 & 19.2 & 9.1 & 14.9 & Other Area hospitals & 7.7 & 5.2 & 2.1 & 7.2 & 20.0 \\
\hline Other Area hospitals & 11.8 & 2.4 & 3.8 & 0.0 & 28.6 & ALL HOSPITALS & 11.4 & 10.2 & 10.0 & 11.9 & 9.6 \\
\hline ALL HOSPITALS & 9.8 & 10.1 & 9.8 & 8.2 & 10.4 & Greater Southern & & & & & \\
\hline \multicolumn{6}{|l|}{ Sydney West } & Goulburn Base & 8.7 & 10.5 & 0.0 & 0.0 & 5.3 \\
\hline Auburn & 6.3 & 4.9 & 4.1 & 7.6 & 12.5 & Moruya & 9.5 & 15.0 & 17.6 & 20.0 & 5.6 \\
\hline Blacktown & 9.9 & 14.2 & 4.3 & 8.7 & 7.1 & Queanbeyan & 0.0 & 11.8 & 0.0 & 25.0 & 5.6 \\
\hline Blue Mountains & 6.3 & 11.1 & 12.0 & 8.7 & 8.7 & Griffith Base & 24.4 & 12.5 & 14.3 & 9.5 & 10.0 \\
\hline Nepean & 6.9 & 5.9 & 10.4 & 9.2 & 6.7 & Wagga Wagga Base & 5.7 & 9.2 & 5.9 & 10.0 & 15.1 \\
\hline Westmead & 11.2 & 8.9 & 8.0 & 8.3 & 5.6 & Calvary, Wagga Wagga & 8.6 & 16.4 & 22.0 & 12.3 & 13.2 \\
\hline The Hills Private & 9.8 & 19.6 & 21.7 & 16.3 & 15.4 & Other Area hospitals & 21.8 & 14.3 & 9.3 & 13.7 & 22.2 \\
\hline Hawkesbury & 11.4 & 12.5 & 16.5 & 10.0 & 15.4 & ALL HOSPITALS & 14.4 & 13.1 & 10.9 & 12.4 & 15.0 \\
\hline Nepean Private & 9.1 & 13.2 & 12.7 & 14.1 & 14.5 & Greater Western & & & & & \\
\hline Westmead Private & 11.3 & 9.0 & 17.5 & 14.8 & 12.9 & Dubbo Base & 9.9 & 11.2 & 8.7 & 14.1 & 10.5 \\
\hline Other Area hospitals & 14.3 & 12.5 & 16.7 & 10.0 & 26.9 & Mudgee & 5.6 & 0.0 & 22.2 & 33.3 & 30.0 \\
\hline ALL HOSPITALS & 9.5 & 10.2 & 10.8 & 10.3 & 9.2 & Bathurst Base & 17.5 & 14.8 & 12.1 & 6.9 & 6.8 \\
\hline \multicolumn{6}{|c|}{ Northern Sydney \& Central Coast } & Orange Base & 18.6 & 17.1 & 9.5 & 7.7 & 8.9 \\
\hline Gosford & 9.5 & 9.9 & 6.2 & 7.2 & 7.7 & Broken Hill Base & 5.7 & 21.7 & 13.6 & 12.9 & 29.2 \\
\hline Wyong & 7.1 & 0.0 & 0.0 & 0.0 & 0.0 & Other Area hospitals & 17.5 & 14.0 & 7.7 & 10.2 & 12.5 \\
\hline Hornsby & 8.2 & 14.8 & 15.2 & 1.9 & 6.6 & ALL HOSPITALS & 13.9 & 13.9 & 10.4 & 11.3 & 12.0 \\
\hline Manly & 9.1 & 12.5 & 4.3 & 8.6 & 8.8 & TOTAL NSW & 10.2 & 10.6 & 9.6 & 9.3 & 9.3 \\
\hline Mona Vale & 14.5 & 2.0 & 5.5 & 9.4 & 17.7 & IUIAL IVSVV & 10.2 & 10.6 & 9.0 & 9.3 & 9.3 \\
\hline Royal North Shore & 6.6 & 12.7 & 6.4 & 8.4 & 4.8 & & & & & & \\
\hline Mater, North Sydney & 9.9 & 11.3 & 10.3 & 12.6 & 8.7 & & & & & & \\
\hline North Shore Private & 15.5 & 13.5 & 9.6 & 11.1 & 13.7 & & & & & & \\
\hline Sydney Adventist & 10.6 & 11.4 & 5.6 & 5.0 & 5.6 & & & & & & \\
\hline North Gosford Private & 18.2 & 19.6 & 22.5 & 18.8 & 14.8 & & & & & & \\
\hline Other Area hospitals & 0.0 & 0.0 & 12.5 & 0.0 & & & & & & & \\
\hline ALL HOSPITALS & 10.7 & 11.8 & 8.5 & 8.8 & 9.0 & & & & & & \\
\hline
\end{tabular}

Source: NSW Midwives Data Collection. Centre for Epidemiology and Research, NSW Health Department.

\# Hospitals with more than 200 deliveries are identified individually. All hospitals include all public and private hospitals. 


\section{Primary caesarean section for failure to progress, Indicator 3.2}

Definition: The number of patients undergoing primary caesarean section for failure to progress after a period of labour with cervical dilation of more than $3 \mathrm{~cm}$ as a percentage of the total number of patients undergoing primary non-elective Caesarean section.
Table 134 shows aggregate information for hospitals where the reported confinements exceeded 200 in 2005, totals for hospitals within each health area, and the NSW total.

\section{TABLE 134}

INDICATOR 3.2: PRIMARY CAESAREAN SECTION FOR FAILURE TO PROGRESS BY HOSPITAL, NSW 2001-2005"

\begin{tabular}{|c|c|c|c|c|c|c|c|c|c|c|c|}
\hline Health Area/ Hospital & $\begin{array}{c}2001 \\
\%\end{array}$ & $\begin{array}{c}2002 \\
\%\end{array}$ & $\begin{array}{c}2003 \\
\%\end{array}$ & $\begin{array}{c}2004 \\
\%\end{array}$ & $\begin{array}{c}2005 \\
\%\end{array}$ & Health Area/ Hospital & $\begin{array}{c}2001 \\
\%\end{array}$ & $\begin{array}{c}2002 \\
\%\end{array}$ & $\begin{array}{c}2003 \\
\%\end{array}$ & $\begin{array}{c}2004 \\
\%\end{array}$ & $\begin{array}{c}2005 \\
\%\end{array}$ \\
\hline \multicolumn{6}{|l|}{ Sydney South West } & \multicolumn{6}{|l|}{ Hunter \& New England } \\
\hline Canterbury & 27.5 & 33.3 & 41.4 & 30.3 & 33.6 & Armidale & 23.5 & 24.3 & 28.6 & 26.7 & 40.9 \\
\hline Royal Prince Alfred & 32.3 & 38.2 & 37.1 & 37.9 & 30.3 & Inverell & 25.0 & 25.0 & 12.5 & 25.0 & 20.0 \\
\hline Fairfield & 16.3 & 30.4 & 24.0 & 39.7 & 31.1 & Moree & 37.5 & 24.0 & 12.5 & 20.0 & 31.8 \\
\hline Liverpool & 33.9 & 30.3 & 28.2 & 36.9 & 35.7 & Tamworth Base & 11.5 & 28.6 & 16.7 & 28.8 & 37.2 \\
\hline Campbelltown & 37.2 & 40.3 & 32.8 & 47.1 & 57.9 & Manning Base & 31.7 & 31.6 & 29.4 & 40.4 & 33.8 \\
\hline Bankstown-Lidcombe & 25.7 & 28.4 & 30.9 & 38.7 & 25.8 & Maitland & 30.1 & 33.3 & 30.8 & 34.1 & 38.2 \\
\hline Sydney Southwest Private & 19.1 & 31.3 & 24.2 & 29.5 & 26.6 & Muswellbrook & 42.9 & 66.7 & 60.0 & 58.8 & 38.1 \\
\hline Bowral & 43.6 & 32.4 & 29.5 & 40.5 & 47.6 & Belmont & 36.1 & 22.0 & 18.5 & 38.6 & 31.3 \\
\hline Other Area hospitals & - & - & 53.5 & 47.6 & 71.4 & John Hunter & 36.6 & 34.7 & 42.3 & 41.4 & 45.3 \\
\hline ALL HOSPITALS & 30.8 & 34.9 & 33.1 & 38.1 & 34.9 & Newcastle Private & - & - & - & 4.3 & 15.4 \\
\hline \multicolumn{6}{|c|}{ South Eastern Sydney \&IIlawarra } & Other Area hospitals & 27.4 & 25.1 & 10.2 & 24.5 & 34.5 \\
\hline Royal Hospital for Women & 40.9 & 41.4 & 49.4 & 56.6 & 57.1 & ALL HOSPITALS & 30.9 & 31.1 & 28.0 & 31.9 & 34.4 \\
\hline St. George & 37.1 & 43.1 & 37.9 & 43.3 & 40.7 & \multicolumn{6}{|l|}{ North Coast } \\
\hline Sutherland & 44.3 & 30.6 & 34.0 & 36.5 & 40.2 & Coffs Harbour & 47.0 & 40.0 & 38.5 & 43.4 & 31.0 \\
\hline Hurstville Community & 26.5 & 31.1 & 34.3 & 38.0 & 27.1 & Grafton Base & 37.2 & 12.5 & 15.4 & 33.9 & 48.9 \\
\hline Kareena Private & 10.1 & 13.6 & 22.2 & 19.6 & 20.0 & Kempsey & 41.2 & 20.0 & 30.8 & 25.0 & 38.5 \\
\hline St. George Private & 29.4 & 26.5 & 25.0 & 25.5 & 24.3 & Lismore Base & 42.6 & 43.1 & 45.6 & 46.4 & 35.9 \\
\hline Prince of Wales Private & 26.7 & 20.6 & 22.5 & 30.5 & 17.9 & Murwillumbah & 31.6 & 34.0 & 42.6 & 25.0 & 39.7 \\
\hline Shoalhaven & 41.2 & 28.9 & 33.3 & 43.2 & 30.4 & Tweed Heads & 23.8 & 37.5 & 30.4 & 21.8 & 31.8 \\
\hline Wollongong & 26.2 & 23.7 & 25.4 & 28.9 & 25.8 & Port Macquarie Base & 30.1 & 21.2 & 31.4 & 32.9 & 45.5 \\
\hline Figtree Private & 38.2 & 33.3 & 20.2 & 20.5 & 22.4 & Other Area hospitals & 29.5 & 22.4 & 34.0 & 31.3 & 60.0 \\
\hline Other Area hospitals & 47.1 & 39.0 & 42.3 & 100.0 & 28.6 & ALL HOSPITALS & 37.3 & 34.5 & 36.2 & 34.4 & 37.6 \\
\hline ALL HOSPITALS & 33.9 & 32.6 & 34.0 & 39.2 & 36.4 & \multicolumn{6}{|l|}{ Greater Southern } \\
\hline Sydney West & & & & & & Goulburn Base & 30.4 & 36.8 & 13.3 & 15.0 & 15.8 \\
\hline Auburn & 20.6 & 37.0 & 20.3 & 30.3 & 33.3 & Moruya & 33.3 & 25.0 & 17.6 & 48.0 & 11.1 \\
\hline Blacktown & 16.5 & 28.4 & 26.1 & 38.6 & 33.3 & Queanbeyan & 40.0 & 17.6 & 44.4 & 31.3 & 50.0 \\
\hline Blue Mountains & 37.5 & 38.9 & 44.0 & 39.1 & 39.1 & Griffith Base & 17.8 & 15.0 & 16.3 & 23.8 & 23.3 \\
\hline Nepean & 43.4 & 38.9 & 48.0 & 46.4 & 44.4 & Wagga Wagga Base & 18.6 & 23.1 & 26.5 & 22.9 & 19.2 \\
\hline Westmead & 36.9 & 35.3 & 35.4 & 39.6 & 48.3 & Calvary, Wagga Wagga & 22.9 & 30.9 & 32.2 & 20.0 & 37.7 \\
\hline The Hills Private & 33.0 & 12.7 & 24.3 & 25.0 & 25.3 & Other Area hospitals & 33.6 & 26.8 & 45.8 & 33.3 & 24.1 \\
\hline Hawkesbury & 45.7 & 36.1 & 40.2 & 41.3 & 36.5 & ALL HOSPITALS & 27.3 & 25.3 & 31.0 & 27.6 & 25.4 \\
\hline Nepean Private & 34.1 & 33.3 & 37.3 & 46.5 & 45.2 & \multicolumn{6}{|l|}{ Greater Western } \\
\hline Westmead Private & 16.3 & 21.5 & 25.7 & 31.4 & 24.9 & Dubbo Base & 24.7 & 23.5 & 26.9 & 24.2 & 23.2 \\
\hline Other Area hospitals & 10.7 & 33.3 & 20.8 & 40.0 & 34.6 & Mudgee & 33.3 & 27.8 & 38.9 & 33.3 & 45.0 \\
\hline ALL HOSPITALS & 31.6 & 32.2 & 34.3 & 39.2 & 39.6 & Bathurst Base & 32.5 & 37.0 & 24.1 & 37.9 & 44.6 \\
\hline \multicolumn{6}{|c|}{ Northern Sydney \& Central Coast } & Orange Base & 25.4 & 35.5 & 20.6 & 23.1 & 17.8 \\
\hline Gosford & 45.2 & 36.8 & 45.0 & 36.5 & 39.3 & Broken Hill Base & 14.3 & 47.8 & 36.4 & 29.0 & 16.7 \\
\hline Wyong & 64.3 & 45.5 & 87.5 & 72.7 & 50.0 & Other Area hospitals & 40.4 & 41.9 & 48.1 & 46.9 & 46.4 \\
\hline Hornsby & 42.5 & 51.9 & 27.3 & 36.9 & 40.8 & ALL HOSPITALS & 28.8 & 33.6 & 30.0 & 30.9 & 30.6 \\
\hline Manly & 50.0 & 33.3 & 43.0 & 37.1 & 38.6 & \multirow{9}{*}{ TOTAL NSW } & \multirow{9}{*}{31.9} & \multirow{9}{*}{31.9} & \multirow{9}{*}{32.9} & \multirow{9}{*}{35.8} & \multirow{9}{*}{35.2} \\
\hline Mona Vale & 36.4 & 38.8 & 41.8 & 43.8 & 34.2 & & & & & & \\
\hline Royal North Shore & 34.2 & 26.9 & 37.0 & 37.3 & 45.7 & & & & & & \\
\hline Mater, North Sydney & 22.8 & 26.8 & 25.7 & 31.8 & 23.7 & & & & & & \\
\hline North Shore Private & 26.8 & 24.3 & 33.2 & 31.1 & 27.2 & & & & & & \\
\hline Sydney Adventist & 18.2 & 16.5 & 12.6 & 6.9 & 7.0 & & & & & & \\
\hline North Gosford Private & 37.5 & 37.4 & 33.8 & 45.0 & 44.3 & & & & & & \\
\hline Other Area hospitals & 31.0 & 23.1 & 29.2 & 44.4 & & & & & & & \\
\hline ALL HOSPITALS & 31.7 & 29.1 & 32.5 & 32.3 & 31.9 & & & & & & \\
\hline
\end{tabular}




\section{Primary caesarean section for fetal} distress, Indicator 4.1

Definition: The number of patients undergoing primary Caesarean section for fetal distress as a percentage of the total number of patients delivering.
Table 135 shows aggregate information for hospitals where the reported confinements exceeded 200 in 2005, totals for hospitals within each health area, and the NSW total.

\section{TABLE 135}

INDICATOR 4.1: PRIMARY CAESAREAN SECTION FOR FETAL DISTRESS BY HOSPITAL, NSW 2001-2005"

\begin{tabular}{|c|c|c|c|c|c|c|c|c|c|c|c|}
\hline Health Area/ Hospital & $\begin{array}{c}2001 \\
\%\end{array}$ & $\begin{array}{c}2002 \\
\%\end{array}$ & $\begin{array}{c}2003 \\
\%\end{array}$ & $\begin{array}{c}2004 \\
\%\end{array}$ & $\begin{array}{c}2005 \\
\%\end{array}$ & Health Area/ Hospital & $\begin{array}{c}2001 \\
\%\end{array}$ & $\begin{array}{c}2002 \\
\%\end{array}$ & $\begin{array}{c}2003 \\
\%\end{array}$ & $\begin{array}{c}2004 \\
\%\end{array}$ & $\begin{array}{c}2005 \\
\%\end{array}$ \\
\hline \multicolumn{6}{|l|}{ Sydney South West } & \multicolumn{6}{|l|}{ Hunter \& New England } \\
\hline Canterbury & 2.9 & 3.1 & 2.7 & 3.2 & 2.8 & Armidale & 2.8 & 2.6 & 0.9 & 3.8 & 2.5 \\
\hline Royal Prince Alfred & 4.4 & 4.3 & 3.5 & 4.0 & 3.5 & Inverell & 1.9 & 2.3 & 2.4 & 2.2 & 2.9 \\
\hline Fairfield & 2.7 & 1.7 & 3.2 & 1.5 & 2.1 & Moree & 3.4 & 3.6 & 0.5 & 2.0 & 2.9 \\
\hline Liverpool & 3.7 & 3.7 & 4.9 & 4.0 & 4.7 & Tamworth Base & 2.5 & 2.4 & 3.1 & 2.4 & 3.3 \\
\hline Campbelltown & 2.1 & 3.2 & 3.0 & 3.0 & 1.6 & Manning Base & 1.5 & 2.5 & 1.9 & 1.9 & 3.0 \\
\hline Bankstown-Lidcombe & 3.7 & 2.5 & 2.5 & 2.5 & 2.5 & Maitland & 4.1 & 5.4 & 4.6 & 3.5 & 4.3 \\
\hline Sydney Southwest Private & 3.5 & 3.4 & 3.7 & 3.3 & 3.7 & Muswellbrook & 1.5 & 2.2 & 0.9 & 1.8 & 2.1 \\
\hline Bowral & 0.6 & 2.0 & 1.2 & 1.7 & 1.9 & Belmont & 1.3 & 4.1 & 3.2 & 2.1 & 2.8 \\
\hline Other Area hospitals & - & - & 1.1 & 1.5 & 1.5 & John Hunter & 3.6 & 3.9 & 3.0 & 3.4 & 3.4 \\
\hline ALL HOSPITALS & 3.3 & 3.2 & 3.3 & 3.2 & 3.1 & Newcastle Private & - & - & - & 3.5 & 4.6 \\
\hline \multicolumn{6}{|c|}{ South Eastern Sydney \&lllawarra } & Other Area hospitals & 2.5 & 2.1 & 2.1 & 2.5 & 1.7 \\
\hline Royal Hospital for Women & 3.3 & 3.5 & 3.9 & 4.1 & 4.3 & ALL HOSPITALS & 2.9 & 3.4 & 2.8 & 3.0 & 3.5 \\
\hline St. George & 4.1 & 4.0 & 3.9 & 4.2 & 5.0 & \multicolumn{6}{|l|}{ North Coast } \\
\hline Sutherland & 2.3 & 2.9 & 3.2 & 3.3 & 1.5 & Coffs Harbour & 0.6 & 0.9 & 1.6 & 2.1 & 2.1 \\
\hline Hurstville Community & 2.7 & 3.6 & 2.8 & 3.5 & 3.5 & Grafton Base & 1.4 & 8.5 & 5.0 & 4.4 & 2.1 \\
\hline Kareena Private & 5.3 & 3.6 & 4.1 & 3.2 & 3.2 & Kempsey & 0.7 & 1.6 & 2.2 & 2.8 & 2.6 \\
\hline St. George Private & 3.4 & 3.0 & 4.1 & 3.3 & 3.6 & Lismore Base & 2.4 & 3.9 & 3.5 & 2.5 & 4.3 \\
\hline Prince of Wales Private & 3.5 & 3.7 & 2.6 & 2.7 & 1.7 & Murwillumbah & 4.0 & 3.8 & 2.2 & 7.0 & 2.0 \\
\hline Shoalhaven & 3.5 & 2.9 & 4.3 & 1.6 & 3.1 & Tweed Heads & 2.5 & 2.6 & 3.3 & 3.5 & 3.4 \\
\hline Wollongong & 4.6 & 4.7 & 3.6 & 3.2 & 3.6 & Port Macquarie Base & 3.9 & 3.1 & 3.9 & 3.8 & 2.7 \\
\hline Figtree Private & 2.9 & 4.7 & 5.7 & 4.9 & 3.1 & Other Area hospitals & 2.3 & 1.7 & 2.4 & 2.4 & 0.0 \\
\hline Other Area hospitals & 1.8 & 2.1 & 1.9 & 0.0 & 1.2 & ALL HOSPITALS & 2.1 & 3.0 & 2.9 & 3.1 & 2.8 \\
\hline ALL HOSPITALS & 3.5 & 3.7 & 3.7 & 3.5 & 3.6 & \multicolumn{6}{|l|}{ Greater Southern } \\
\hline Sydney West & & & & & & Goulburn Base & 0.9 & 2.3 & 3.3 & 1.1 & 1.4 \\
\hline Auburn & 3.1 & 3.1 & 3.9 & 2.2 & 2.5 & Moruya & 1.8 & 3.9 & 3.3 & 1.2 & 1.5 \\
\hline Blacktown & 5.7 & 4.7 & 5.9 & 4.5 & 4.5 & Queanbeyan & 4.0 & 1.6 & 1.6 & 0.8 & 1.4 \\
\hline Blue Mountains & 3.7 & 3.5 & 2.2 & 2.4 & 5.2 & Griffith Base & 3.3 & 3.3 & 4.7 & 4.0 & 4.1 \\
\hline Nepean & 3.9 & 4.6 & 4.6 & 4.8 & 5.1 & Wagga Wagga Base & 4.0 & 3.0 & 4.0 & 4.4 & 4.4 \\
\hline Westmead & 5.7 & 5.0 & 5.9 & 6.8 & 6.2 & Calvary, Wagga Wagga & 1.8 & 3.6 & 1.2 & 3.5 & 1.4 \\
\hline The Hills Private & 3.0 & 2.9 & 3.3 & 2.3 & 2.8 & Other Area hospitals & 1.6 & 1.7 & 1.8 & 2.1 & 1.6 \\
\hline Hawkesbury & 1.6 & 2.1 & 3.7 & 3.4 & 4.2 & ALL HOSPITALS & 2.4 & 2.5 & 2.6 & 2.7 & 2.3 \\
\hline Nepean Private & 2.1 & 2.5 & 3.6 & 2.0 & 3.1 & \multicolumn{6}{|l|}{ Greater Western } \\
\hline Westmead Private & 3.3 & 2.7 & 3.1 & 4.6 & 2.9 & Dubbo Base & 2.7 & 2.1 & 2.5 & 3.5 & 3.2 \\
\hline Other Area hospitals & 6.5 & 2.8 & 6.7 & 3.4 & 2.9 & Mudgee & 3.2 & 2.8 & 1.0 & 0.5 & 0.4 \\
\hline ALL HOSPITALS & 4.2 & 4.0 & 4.7 & 4.6 & 4.5 & Bathurst Base & 2.3 & 4.1 & 3.5 & 2.5 & 2.1 \\
\hline \multicolumn{6}{|c|}{ Northern Sydney \& Central Coast } & Orange Base & 2.9 & 2.5 & 2.1 & 3.3 & 4.7 \\
\hline Gosford & 2.8 & 3.5 & 3.6 & 5.9 & 4.4 & Broken Hill Base & 3.0 & 0.8 & 1.5 & 3.5 & 2.2 \\
\hline Wyong & 0.8 & 1.5 & 0.6 & 0.0 & 0.0 & Other Area hospitals & 1.4 & 1.0 & 1.9 & 2.3 & 1.1 \\
\hline Hornsby & 3.1 & 3.0 & 3.7 & 4.4 & 2.6 & ALL HOSPITALS & 2.5 & 2.2 & 2.3 & 2.9 & 2.7 \\
\hline Manly & 2.0 & 2.7 & 2.7 & 3.8 & 3.6 & \multirow{9}{*}{ TOTAL NSW } & \multirow{9}{*}{3.3} & \multirow{9}{*}{3.4} & \multirow{9}{*}{3.5} & \multirow{9}{*}{3.5} & \multirow{9}{*}{3.4} \\
\hline Mona Vale & 2.2 & 2.2 & 3.3 & 2.6 & 2.0 & & & & & & \\
\hline Royal North Shore & 3.4 & 3.8 & 6.6 & 4.7 & 3.8 & & & & & & \\
\hline Mater, North Sydney & 4.5 & 4.1 & 4.2 & 3.6 & 2.9 & & & & & & \\
\hline North Shore Private & 2.6 & 3.7 & 3.7 & 3.7 & 3.4 & & & & & & \\
\hline Sydney Adventist & 3.2 & 3.0 & 2.3 & 2.3 & 2.9 & & & & & & \\
\hline North Gosford Private & 2.4 & 2.0 & 1.3 & 1.8 & 1.6 & & & & & & \\
\hline Other Area hospitals & 3.0 & 4.2 & 2.3 & 0.8 & 0.0 & & & & & & \\
\hline ALL HOSPITALS & 3.0 & 3.3 & 3.5 & 3.7 & 3.2 & & & & & & \\
\hline
\end{tabular}




\section{Primary caesarean section for fetal distress, Indicator $\mathbf{4 . 2}$}

Definition: The number of patients undergoing primary caesarean section for fetal distress as a percentage of the total number of patients delivering by primary caesarean section.
Table 136 shows aggregate information for hospitals where the reported confinements exceeded 200 in 2005, totals for hospitals within each health area, and the NSW total.

\section{TABLE 136}

INDICATOR 4.2: PRIMARY CAESAREAN SECTION FOR FETAL DISTRESS BY HOSPITAL, NSW 2001-2005"

\begin{tabular}{|c|c|c|c|c|c|c|c|c|c|c|c|}
\hline Health Area/ Hospital & $\begin{array}{c}2001 \\
\%\end{array}$ & $\begin{array}{c}2002 \\
\%\end{array}$ & $\begin{array}{c}2003 \\
\%\end{array}$ & $\begin{array}{c}2004 \\
\%\end{array}$ & $\begin{array}{c}2005 \\
\%\end{array}$ & Health Area/ Hospital & $\begin{array}{c}2001 \\
\%\end{array}$ & $\begin{array}{c}2002 \\
\%\end{array}$ & $\begin{array}{c}2003 \\
\%\end{array}$ & $\begin{array}{c}2004 \\
\%\end{array}$ & $\begin{array}{c}2005 \\
\%\end{array}$ \\
\hline \multicolumn{6}{|l|}{ Sydney South West } & \multicolumn{6}{|l|}{ Hunter \& New England } \\
\hline Canterbury & 26.0 & 25.3 & 19.3 & 23.8 & 20.5 & Armidale & 16.5 & 21.4 & 9.5 & 20.0 & 15.6 \\
\hline Royal Prince Alfred & 23.7 & 21.0 & 17.6 & 20.0 & 15.9 & Inverell & 11.5 & 18.5 & 14.7 & 12.1 & 15.8 \\
\hline Fairfield & 25.1 & 17.8 & 30.8 & 17.2 & 16.7 & Moree & 25.0 & 22.2 & 5.9 & 21.1 & 21.9 \\
\hline Liverpool & 24.2 & 29.1 & 32.2 & 21.6 & 27.3 & Tamworth Base & 15.8 & 19.4 & 21.2 & 16.5 & 16.3 \\
\hline Campbelltown & 15.9 & 23.8 & 19.4 & 16.1 & 12.5 & Manning Base & 11.9 & 17.7 & 13.9 & 12.8 & 19.4 \\
\hline Bankstown-Lidcombe & 28.9 & 23.1 & 20.7 & 18.6 & 23.6 & Maitland & 26.7 & 25.8 & 21.9 & 18.2 & 25.8 \\
\hline Sydney Southwest Private & 18.4 & 19.7 & 20.0 & 18.8 & 19.8 & Muswellbrook & 16.7 & 14.7 & 8.7 & 13.6 & 13.8 \\
\hline Bowral & 4.7 & 18.2 & 9.7 & 16.2 & 17.9 & Belmont & 12.5 & 22.6 & 23.2 & 13.4 & 30.0 \\
\hline Other Area hospitals & - & - & 12.2 & 25.9 & 14.3 & John Hunter & 22.2 & 21.7 & 16.9 & 18.0 & 18.5 \\
\hline ALL HOSPITALS & 22.5 & 22.8 & 21.6 & 19.7 & 18.8 & Newcastle Private & - & - & - & 13.3 & 20.6 \\
\hline \multicolumn{6}{|c|}{ South Eastern Sydney \&IIlawarra } & Other Area hospitals & 15.2 & 13.4 & 11.7 & 15.9 & 15.1 \\
\hline Royal Hospital for Women & 16.1 & 16.9 & 17.7 & 17.2 & 18.0 & ALL HOSPITALS & 18.7 & 19.7 & 16.3 & 16.4 & 19.8 \\
\hline St. George & 26.0 & 25.7 & 21.8 & 24.8 & 27.7 & \multicolumn{6}{|l|}{ North Coast } \\
\hline Sutherland & 13.6 & 16.9 & 20.5 & 18.0 & 9.7 & Coffs Harbour & 3.6 & 5.4 & 8.1 & 13.6 & 11.6 \\
\hline Hurstville Community & 13.1 & 14.7 & 12.3 & 14.5 & 16.7 & Grafton Base & 6.4 & 41.9 & 25.7 & 20.7 & 10.7 \\
\hline Kareena Private & 17.4 & 13.4 & 14.3 & 13.2 & 12.7 & Kempsey & 9.1 & 19.0 & 18.2 & 31.8 & 20.0 \\
\hline St. George Private & 14.3 & 13.7 & 17.6 & 13.4 & 13.4 & Lismore Base & 15.2 & 19.0 & 17.4 & 13.2 & 20.2 \\
\hline Prince of Wales Private & 15.8 & 15.6 & 10.2 & 11.3 & 6.3 & Murwillumbah & 27.1 & 18.6 & 10.8 & 28.9 & 8.0 \\
\hline Shoalhaven & 19.0 & 16.9 & 18.8 & 9.1 & 14.1 & Tweed Heads & 20.8 & 17.5 & 19.5 & 21.9 & 20.5 \\
\hline Wollongong & 27.4 & 29.6 & 21.1 & 18.3 & 21.3 & Port Macquarie Base & 20.8 & 18.0 & 19.5 & 20.9 & 16.4 \\
\hline Figtree Private & 16.2 & 25.6 & 28.3 & 28.6 & 18.9 & Other Area hospitals & 18.0 & 14.1 & 17.4 & 19.4 & 0.0 \\
\hline Other Area hospitals & 13.0 & 15.1 & 12.5 & 0.0 & 8.3 & ALL HOSPITALS & 14.4 & 18.1 & 16.8 & 19.0 & 15.9 \\
\hline ALL HOSPITALS & 18.0 & 18.4 & 17.5 & 17.0 & 16.4 & \multicolumn{6}{|l|}{ Greater Southern } \\
\hline Sydney West & & & & & & Goulburn Base & 3.8 & 12.0 & 13.3 & 4.8 & 9.3 \\
\hline Auburn & 31.1 & 27.9 & 38.6 & 22.0 & 25.7 & Moruya & 7.7 & 16.7 & 20.8 & 5.4 & 12.9 \\
\hline Blacktown & 38.6 & 31.5 & 35.9 & 27.3 & 28.4 & Queanbeyan & 31.0 & 14.3 & 15.8 & 7.7 & 13.3 \\
\hline Blue Mountains & 19.6 & 21.2 & 12.5 & 17.1 & 37.8 & Griffith Base & 20.0 & 15.9 & 27.3 & 22.7 & 25.9 \\
\hline Nepean & 20.0 & 24.3 & 21.4 & 20.3 & 25.0 & Wagga Wagga Base & 25.4 & 20.2 & 26.4 & 25.2 & 22.9 \\
\hline Westmead & 31.7 & 26.9 & 27.1 & 30.5 & 26.3 & Calvary, Wagga Wagga & 10.7 & 18.0 & 5.9 & 13.4 & 7.9 \\
\hline The Hills Private & 17.3 & 20.7 & 20.6 & 14.8 & 19.0 & Other Area hospitals & 13.4 & 14.4 & 14.5 & 15.2 & 11.1 \\
\hline Hawkesbury & 13.5 & 14.4 & 19.3 & 15.8 & 19.0 & ALL HOSPITALS & 16.5 & 16.2 & 16.8 & 15.8 & 14.6 \\
\hline Nepean Private & 11.9 & 11.0 & 12.7 & 9.8 & 14.7 & \multicolumn{6}{|l|}{ Greater Western } \\
\hline Westmead Private & 15.5 & 13.2 & 14.1 & 20.8 & 12.2 & Dubbo Base & 19.7 & 13.9 & 19.7 & 23.7 & 23.4 \\
\hline Other Area hospitals & 31.9 & 12.8 & 39.4 & 29.2 & 16.7 & Mudgee & 26.1 & 28.6 & 8.0 & 8.3 & 3.0 \\
\hline ALL HOSPITALS & 25.0 & 22.9 & 24.1 & 23.0 & 22.9 & Bathurst Base & 10.9 & 17.4 & 21.1 & 13.3 & 9.6 \\
\hline \multicolumn{6}{|c|}{ Northern Sydney \& Central Coast } & Orange Base & 17.0 & 12.1 & 13.8 & 22.0 & 24.3 \\
\hline Gosford & 16.2 & 19.4 & 19.5 & 27.0 & 21.4 & Broken Hill Base & 17.4 & 3.3 & 8.9 & 13.3 & 17.6 \\
\hline Wyong & 18.8 & 35.7 & 16.7 & 0.0 & 0.0 & Other Area hospitals & 11.7 & 7.4 & 13.4 & 14.5 & 8.8 \\
\hline Hornsby & 20.0 & 17.5 & 24.0 & 24.1 & 12.7 & ALL HOSPITALS & 15.9 & 13.4 & 16.1 & 18.8 & 17.4 \\
\hline Manly & 12.6 & 16.4 & 14.7 & 22.9 & 17.5 & \multirow{9}{*}{ TOTAL NSW } & \multirow{9}{*}{19.2} & \multirow{9}{*}{19.1} & \multirow{9}{*}{18.9} & \multirow{9}{*}{18.6} & \multirow{9}{*}{17.8} \\
\hline Mona Vale & 15.7 & 14.8 & 20.2 & 17.0 & 11.3 & & & & & & \\
\hline Royal North Shore & 16.2 & 16.7 & 24.5 & 18.6 & 16.6 & & & & & & \\
\hline Mater, North Sydney & 16.8 & 14.0 & 14.9 & 13.9 & 11.1 & & & & & & \\
\hline North Shore Private & 10.0 & 12.9 & 14.2 & 13.5 & 12.0 & & & & & & \\
\hline Sydney Adventist & 17.7 & 15.7 & 12.7 & 11.8 & 13.8 & & & & & & \\
\hline North Gosford Private & 10.1 & 8.5 & 5.5 & 8.3 & 7.4 & & & & & & \\
\hline Other Area hospitals & 30.9 & 30.8 & 20.4 & 11.8 & & & & & & & \\
\hline ALL HOSPITALS & 15.4 & 15.4 & 16.3 & 16.8 & 14.1 & & & & & & \\
\hline
\end{tabular}




\section{Intact lower genital tract in primiparous patients delivering vaginally, Indicator 5.1}

Definition: The number of primiparous patients not requiring surgical repair of the lower genital tract as a percentage of the total number of primiparous patients delivering vaginally.
Table 137 shows aggregate information for hospitals where the reported confinements exceeded 200 in 2005, totals for hospitals within each health area, and the NSW total.

\section{TABLE 137}

INDICATOR 5.1: INTACT LOWER GENITAL TRACT IN PRIMIPAROUS PATIENTS DELIVERING VAGINALLY BY HOSPITAL, NSW 2001-2005\#

\begin{tabular}{|c|c|c|c|c|c|c|c|c|c|c|c|}
\hline Health Area/ Hospital & $\begin{array}{c}2001 \\
\%\end{array}$ & $\begin{array}{c}2002 \\
\%\end{array}$ & $\begin{array}{c}2003 \\
\%\end{array}$ & $\begin{array}{c}2004 \\
\%\end{array}$ & $\begin{array}{c}2005 \\
\%\end{array}$ & Health Area/ Hospital & $\begin{array}{c}2001 \\
\%\end{array}$ & $\begin{array}{c}2002 \\
\%\end{array}$ & $\begin{array}{c}2003 \\
\%\end{array}$ & $\begin{array}{c}2004 \\
\%\end{array}$ & $\begin{array}{c}2005 \\
\%\end{array}$ \\
\hline \multicolumn{6}{|l|}{ Sydney South West } & \multicolumn{6}{|l|}{ Hunter \& New England } \\
\hline Canterbury & 28.4 & 28.9 & 31.2 & 24.4 & 23.3 & Armidale & 16.5 & 21.2 & 22.6 & 14.3 & 22.0 \\
\hline Royal Prince Alfred & 29.3 & 28.0 & 31.0 & 31.8 & 31.7 & Inverell & 39.2 & 48.9 & 23.3 & 19.4 & 24.1 \\
\hline Fairfield & 31.2 & 31.1 & 33.9 & 31.3 & 29.6 & Moree & 55.8 & 53.4 & 50.0 & 51.0 & 42.6 \\
\hline Liverpool & 28.1 & 27.0 & 23.7 & 27.0 & 25.3 & Tamworth Base & 23.6 & 46.5 & 42.9 & 34.6 & 28.8 \\
\hline Campbelltown & 28.6 & 30.5 & 28.8 & 29.5 & 29.3 & Manning Base & 31.8 & 36.7 & 42.0 & 32.8 & 39.6 \\
\hline Bankstown-Lidcombe & 25.1 & 26.7 & 24.7 & 22.6 & 26.8 & Maitland & 43.0 & 39.2 & 35.7 & 39.3 & 34.8 \\
\hline Sydney Southwest Private & 25.4 & 17.4 & 26.7 & 20.9 & 20.7 & Muswellbrook & 42.0 & 53.2 & 36.0 & 42.0 & 31.6 \\
\hline Bowral & 40.1 & 44.5 & 27.2 & 40.5 & 35.9 & Belmont & 43.4 & 38.7 & 45.6 & 47.2 & 40.6 \\
\hline Other Area hospitals & - & - & 56.3 & 58.4 & 68.8 & John Hunter & 41.4 & 38.3 & 38.5 & 27.8 & 34.6 \\
\hline ALL HOSPITALS & 28.9 & 28.5 & 29.7 & 29.7 & 28.6 & Newcastle Private & - & - & - & 30.1 & 33.3 \\
\hline \multicolumn{6}{|c|}{ South Eastern Sydney \&IIlawarra } & Other Area hospitals & 36.6 & 35.0 & 36.1 & 38.4 & 38.0 \\
\hline Royal Hospital for Women & 23.9 & 24.4 & 25.9 & 28.8 & 27.3 & ALL HOSPITALS & 38.3 & 38.4 & 37.7 & 33.3 & 34.2 \\
\hline St. George & 30.7 & 34.4 & 38.5 & 28.8 & 33.2 & \multicolumn{6}{|l|}{ North Coast } \\
\hline Sutherland & 27.8 & 28.7 & 27.6 & 24.9 & 22.9 & Coffs Harbour & 31.6 & 41.3 & 42.7 & 31.2 & 37.7 \\
\hline Hurstville Community & 15.4 & 14.4 & 12.0 & 10.4 & 5.7 & Grafton Base & 29.6 & 46.8 & 45.0 & 36.7 & 46.8 \\
\hline Kareena Private & 20.7 & 23.2 & 23.2 & 23.8 & 31.4 & Kempsey & 44.8 & 55.2 & 58.6 & 54.3 & 45.6 \\
\hline St. George Private & 17.3 & 17.7 & 23.9 & 17.9 & 19.4 & Lismore Base & 38.9 & 35.1 & 35.0 & 22.5 & 28.4 \\
\hline Prince of Wales Private & 13.5 & 13.3 & 12.5 & 14.5 & 18.9 & Murwillumbah & 22.1 & 25.9 & 18.3 & 29.6 & 17.4 \\
\hline Shoalhaven & 34.2 & 33.8 & 32.5 & 33.9 & 30.9 & Tweed Heads & 34.5 & 47.4 & 40.0 & 34.1 & 40.0 \\
\hline Wollongong & 35.5 & 29.6 & 27.4 & 21.9 & 26.8 & Port Macquarie Base & 26.4 & 23.0 & 36.4 & 36.9 & 42.0 \\
\hline Figtree Private & 19.7 & 14.1 & 10.6 & 13.8 & 26.0 & Other Area hospitals & 36.3 & 27.9 & 35.5 & 38.6 & 38.2 \\
\hline Other Area hospitals & 29.5 & 32.2 & 25.0 & 57.1 & 35.0 & ALL HOSPITALS & 35.1 & 37.9 & 38.3 & 33.1 & 37.0 \\
\hline ALL HOSPITALS & 25.2 & 25.1 & 25.8 & 24.1 & 26.1 & \multicolumn{6}{|l|}{ Greater Southern } \\
\hline Sydney West & & & & & & Goulburn Base & 30.2 & 27.8 & 17.9 & 11.4 & 8.1 \\
\hline Auburn & 28.9 & 29.7 & 35.8 & 36.5 & 33.6 & Moruya & 52.0 & 66.7 & 54.5 & 46.2 & 59.1 \\
\hline Blacktown & 22.5 & 31.2 & 25.3 & 25.9 & 19.2 & Queanbeyan & 66.7 & 67.8 & 67.3 & 66.1 & 65.8 \\
\hline Blue Mountains & 43.0 & 46.9 & 51.5 & 35.3 & 48.2 & Griffith Base & 66.0 & 63.4 & 64.7 & 49.4 & 59.6 \\
\hline Nepean & 38.6 & 42.1 & 34.3 & 35.2 & 30.7 & Wagga Wagga Base & 47.1 & 43.3 & 44.6 & 42.3 & 38.4 \\
\hline Westmead & 27.2 & 27.7 & 23.4 & 18.5 & 18.9 & Calvary, Wagga Wagga & 32.3 & 27.3 & 27.0 & 22.2 & 21.3 \\
\hline The Hills Private & 18.8 & 24.6 & 23.5 & 14.8 & 22.3 & Other Area hospitals & 42.6 & 45.5 & 40.8 & 50.3 & 40.2 \\
\hline Hawkesbury & 46.8 & 47.6 & 55.3 & 47.3 & 50.9 & ALL HOSPITALS & 47.3 & 46.5 & 44.2 & 45.3 & 42.7 \\
\hline Nepean Private & 19.7 & 18.5 & 14.7 & 12.7 & 10.3 & \multicolumn{6}{|l|}{ Greater Western } \\
\hline Westmead Private & 26.5 & 18.9 & 17.1 & 15.5 & 15.6 & Dubbo Base & 34.5 & 39.0 & 34.4 & 29.7 & 33.9 \\
\hline Other Area hospitals & 13.5 & 25.0 & 26.3 & 30.0 & 38.8 & Mudgee & 40.9 & 29.8 & 33.3 & 24.4 & 36.0 \\
\hline ALL HOSPITALS & 29.5 & 31.2 & 28.3 & 25.5 & 24.3 & Bathurst Base & 43.4 & 42.4 & 36.9 & 36.0 & 40.3 \\
\hline \multicolumn{6}{|c|}{ Northern Sydney \& Central Coast } & Orange Base & 28.8 & 31.4 & 33.3 & 37.0 & 50.8 \\
\hline Gosford & 25.9 & 30.4 & 33.3 & 29.4 & 26.7 & Broken Hill Base & 65.1 & 63.2 & 58.3 & 51.7 & 62.3 \\
\hline Wyong & 50.0 & 45.3 & 42.0 & 34.0 & 49.1 & Other Area hospitals & 50.0 & 39.3 & 49.5 & 40.0 & 39.0 \\
\hline Hornsby & 15.1 & 14.0 & 18.6 & 20.5 & 17.9 & ALL HOSPITALS & 40.5 & 39.2 & 38.6 & 35.2 & 41.0 \\
\hline Manly & 27.2 & 22.1 & 21.4 & 18.9 & 27.0 & \multirow{9}{*}{ TOTAL NSW } & \multirow{9}{*}{29.7} & \multirow{9}{*}{29.8} & \multirow{9}{*}{29.6} & \multirow{9}{*}{27.9} & \multirow{9}{*}{28.6} \\
\hline Mona Vale & 26.3 & 41.4 & 30.9 & 36.8 & 42.5 & & & & & & \\
\hline Royal North Shore & 20.7 & 15.6 & 20.0 & 21.3 & 23.0 & & & & & & \\
\hline Mater, North Sydney & 11.0 & 9.7 & 11.6 & 14.3 & 16.6 & & & & & & \\
\hline North Shore Private & 12.3 & 7.5 & 10.7 & 13.0 & 13.8 & & & & & & \\
\hline Sydney Adventist & 12.5 & 11.5 & 12.9 & 12.5 & 10.8 & & & & & & \\
\hline North Gosford Private & 14.6 & 19.2 & 21.5 & 14.2 & 23.2 & & & & & & \\
\hline Other Area hospitals & 21.5 & 19.3 & 20.0 & 34.2 & 44.4 & & & & & & \\
\hline ALL HOSPITALS & 19.0 & 17.9 & 20.2 & 20.1 & 21.6 & & & & & & \\
\hline
\end{tabular}




\section{Apgar scores, Indicator 6.1}

Definition: The number of babies born with an Apgar score of four or below at five minutes post delivery as a percentage of the total number of babies born.
Table 138 shows aggregate information for hospitals where the reported confinements exceeded 200 in 2005, totals for hospitals within each health area, and the NSW total.

\section{TABLE 138}

INDICATOR 6.1: APGAR SCORE OF 4 OR LESS BY HOSPITAL, NSW 2001-2005"

\begin{tabular}{|c|c|c|c|c|c|c|c|c|c|c|c|}
\hline Health Area/ Hospital & $\begin{array}{c}2001 \\
\%\end{array}$ & $\begin{array}{c}2002 \\
\%\end{array}$ & $\begin{array}{c}2003 \\
\%\end{array}$ & $\begin{array}{c}2004 \\
\%\end{array}$ & $\begin{array}{c}2005 \\
\%\end{array}$ & Health Area/ Hospital & $\begin{array}{c}2001 \\
\%\end{array}$ & $\begin{array}{c}2002 \\
\%\end{array}$ & $\begin{array}{c}2003 \\
\%\end{array}$ & $\begin{array}{c}2004 \\
\%\end{array}$ & $\begin{array}{c}2005 \\
\%\end{array}$ \\
\hline \multicolumn{6}{|l|}{ Sydney South West } & \multicolumn{6}{|l|}{ Hunter \& New England } \\
\hline Canterbury & 0.9 & 1.2 & 1.1 & 0.8 & 0.7 & Armidale & 1.3 & 0.6 & 1.3 & 0.9 & 1.0 \\
\hline Royal Prince Alfred & 1.5 & 1.6 & 1.4 & 1.6 & 1.7 & Inverell & 0.8 & 0.0 & 0.5 & 0.9 & 0.8 \\
\hline Fairfield & 0.9 & 1.3 & 1.0 & 1.8 & 0.9 & Moree & 2.1 & 1.2 & 2.2 & 2.0 & 2.5 \\
\hline Liverpool & 1.6 & 1.4 & 1.5 & 2.1 & 1.5 & Tamworth Base & 1.8 & 2.0 & 1.8 & 1.7 & 1.8 \\
\hline Campbelltown & 1.3 & 1.3 & 1.4 & 1.0 & 1.2 & Manning Base & 1.5 & 1.4 & 2.0 & 1.4 & 1.0 \\
\hline Bankstown-Lidcombe & 1.4 & 1.1 & 1.0 & 1.1 & 1.0 & Maitland & 0.9 & 0.8 & 0.8 & 0.9 & 1.4 \\
\hline Sydney Southwest Private & 0.7 & 0.4 & 0.3 & 0.7 & 0.6 & Muswellbrook & 0.0 & 0.0 & 0.0 & 0.9 & 0.4 \\
\hline Bowral & 1.0 & 0.8 & 0.8 & 0.9 & 0.6 & Belmont & 0.3 & 0.9 & 0.6 & 0.5 & 0.6 \\
\hline Other Area hospitals & - & - & 0.9 & 0.0 & 0.0 & John Hunter & 1.9 & 1.9 & 2.3 & 2.1 & 2.4 \\
\hline ALL HOSPITALS & 1.3 & 1.3 & 1.2 & 1.4 & 1.2 & Newcastle Private & - & - & - & 0.4 & 1.4 \\
\hline \multicolumn{6}{|c|}{ South Eastern Sydney\&IIlawarra } & Other Area hospitals & 0.7 & 0.7 & 0.4 & 0.7 & 0.8 \\
\hline Royal Hospital for Women & 1.3 & 1.8 & 1.4 & 1.6 & 1.6 & ALL HOSPITALS & 1.3 & 1.2 & 1.4 & 1.3 & 1.6 \\
\hline St. George & 1.2 & 1.2 & 1.2 & 1.2 & 1.0 & \multicolumn{6}{|l|}{ North Coast } \\
\hline Sutherland & 0.7 & 0.1 & 0.6 & 0.2 & 0.5 & Coffs Harbour & 1.2 & 0.6 & 0.9 & 0.9 & 0.6 \\
\hline Hurstville Community & 0.7 & 0.0 & 0.3 & 0.6 & 0.3 & Grafton Base & 1.4 & 1.0 & 1.2 & 0.8 & 0.7 \\
\hline Kareena Private & 0.7 & 0.1 & 0.4 & 0.5 & 0.5 & Kempsey & 2.5 & 0.0 & 1.8 & 0.3 & 1.7 \\
\hline St. George Private & 0.3 & 0.6 & 0.5 & 0.4 & 0.4 & Lismore Base & 1.2 & 1.4 & 1.7 & 1.3 & 0.9 \\
\hline Prince of Wales Private & 0.5 & 0.6 & 0.2 & 0.4 & 0.6 & Murwillumbah & 1.0 & 0.2 & 0.8 & 1.0 & 0.2 \\
\hline Shoalhaven & 0.7 & 0.6 & 1.4 & 1.8 & 1.5 & Tweed Heads & 1.9 & 1.3 & 1.5 & 1.2 & 0.7 \\
\hline Wollongong & 1.1 & 1.0 & 0.7 & 0.5 & 1.2 & Port Macquarie Base & 1.1 & 1.0 & 1.4 & 0.5 & 1.0 \\
\hline Figtree Private & 0.4 & 0.6 & 0.2 & 0.4 & 0.2 & Other Area hospitals & 0.6 & 0.9 & 1.2 & 0.5 & 0.5 \\
\hline Other Area hospitals & 0.4 & 0.0 & 0.8 & 2.1 & 4.7 & ALL HOSPITALS & 1.2 & 0.9 & 1.3 & 0.9 & 0.8 \\
\hline ALL HOSPITALS & 0.9 & 0.9 & 0.8 & 0.9 & 1.0 & \multicolumn{6}{|l|}{ Greater Southern } \\
\hline Sydney West & & & & & & Goulburn Base & 0.6 & 1.3 & 2.3 & 0.4 & 1.7 \\
\hline Auburn & 1.3 & 0.7 & 1.3 & 1.2 & 1.2 & Moruya & 2.9 & 0.6 & 1.6 & 1.6 & 0.9 \\
\hline Blacktown & 1.2 & 1.2 & 1.1 & 0.7 & 0.7 & Queanbeyan & 1.5 & 0.4 & 0.4 & 0.4 & 0.4 \\
\hline Blue Mountains & 1.3 & 0.0 & 0.9 & 0.0 & 0.4 & Griffith Base & 1.8 & 1.4 & 1.3 & 0.7 & 0.7 \\
\hline Nepean & 1.3 & 1.2 & 1.0 & 0.9 & 1.5 & Wagga Wagga Base & 2.0 & 2.2 & 1.1 & 1.8 & 1.8 \\
\hline Westmead & 2.2 & 1.8 & 1.8 & 2.1 & 1.7 & Calvary, Wagga Wagga & 0.4 & 1.0 & 0.3 & 1.9 & 0.5 \\
\hline The Hills Private & 0.3 & 0.6 & 0.3 & 0.7 & 0.5 & Other Area hospitals & 0.4 & 0.4 & 0.5 & 0.2 & 0.5 \\
\hline Hawkesbury & 1.1 & 0.4 & 0.9 & 0.5 & 0.7 & ALL HOSPITALS & 1.1 & 1.0 & 0.9 & 0.9 & 0.9 \\
\hline Nepean Private & 0.4 & 0.4 & 0.7 & 0.3 & 0.9 & \multicolumn{6}{|l|}{ Greater Western } \\
\hline Westmead Private & 0.7 & 0.9 & 0.6 & 0.9 & 0.6 & Dubbo Base & 1.1 & 1.4 & 2.0 & 1.1 & 1.8 \\
\hline Other Area hospitals & 0.0 & 0.5 & 0.5 & 2.5 & 1.2 & Mudgee & 0.5 & 0.0 & 0.5 & 1.1 & 0.0 \\
\hline ALL HOSPITALS & 1.3 & 1.1 & 1.1 & 1.1 & 1.1 & Bathurst Base & 0.3 & 0.6 & 0.5 & 1.7 & 1.0 \\
\hline \multicolumn{6}{|c|}{ Northern Sydney \& CentralCoast } & Orange Base & 1.1 & 1.2 & 0.7 & 2.1 & 0.3 \\
\hline Gosford & 0.8 & 1.1 & 0.5 & 0.9 & 0.9 & Broken Hill Base & 1.6 & 0.8 & 1.1 & 0.8 & 0.7 \\
\hline Wyong & 0.3 & 0.3 & 0.0 & 0.4 & 0.4 & Other Area hospitals & 0.8 & 1.3 & 0.6 & 1.0 & 1.1 \\
\hline Hornsby & 0.6 & 0.9 & 0.8 & 1.0 & 0.8 & ALL HOSPITALS & 0.9 & 1.1 & 1.1 & 1.4 & 1.0 \\
\hline Manly & 1.0 & 1.1 & 0.8 & 0.6 & 0.9 & \multirow{9}{*}{ TOTAL NSW } & \multirow{9}{*}{1.1} & \multirow[t]{9}{*}{1.0} & \multirow{9}{*}{1.0} & \multirow{9}{*}{1.1} & \multirow[t]{9}{*}{1.1} \\
\hline Mona Vale & 0.8 & 0.7 & 0.3 & 0.5 & 0.2 & & & & & & \\
\hline Royal North Shore & 1.5 & 1.0 & 1.3 & 1.5 & 1.3 & & & & & & \\
\hline Mater, North Sydney & 0.4 & 0.7 & 0.4 & 0.4 & 0.5 & & & & & & \\
\hline North Shore Private & 0.8 & 0.7 & 1.0 & 0.6 & 0.7 & & & & & & \\
\hline Sydney Adventist & 0.3 & 0.7 & 0.8 & 0.6 & 0.4 & & & & & & \\
\hline North Gosford Private & 0.8 & 0.4 & 0.2 & 0.4 & 0.5 & & & & & & \\
\hline Other Area hospitals & 0.7 & 1.0 & 1.4 & 0.0 & 0.8 & & & & & & \\
\hline ALL HOSPITALS & 0.7 & 0.8 & 0.7 & 0.8 & 0.7 & & & & & & \\
\hline
\end{tabular}

